\title{
1 Methylation of KRAS at lysine 182 and 184 by SETD7 promotes
}

\section{KRAS degradation}

3 Chengyao Chiang ${ }^{1,5}$, Tian Xiao, ${ }^{1,5}$, Songqing Fan $^{2}$, Hongmei Zheng ${ }^{2}$, Shuaihu $\mathrm{Li}^{1}$,

4 Wenjun Guo ${ }^{1}$, Min Zhang ${ }^{1}$, Chuanqi Zhong ${ }^{3}$, Juan Zeng ${ }^{4}$, Duo Zheng ${ }^{1, *}$

$5 \quad{ }^{1}$ Guangdong Provincial Key Laboratory of Regional Immunity and Diseases, Shenzhen

6 University International Cancer Center, Department of Cell Biology and Genetics,

7 School of Medicine, Shenzhen University, Shenzhen, Guangdong, China.

$8{ }^{2}$ Department of Pathology, The Second Xiangya Hospital, Central South University, 9 Changsha, Hunan, China.

$10{ }^{3}$ State Key Laboratory of Cellular Stress Biology, Innovation Center for Cell Signaling 11 Network, School of Life Sciences, Xiamen University, Xiamen, Fujian, China.

$12{ }^{4}$ School of Biomedical Engineering, Guangdong Medical University, Dongguan, 13 Guangdong, China

$14{ }^{5}$ The authors contributed equally to this work.

15 *Correspondence to Tian Xiao, Department of Cell Biology and Genetics, Shenzhen 16 University Health Sciences Center, 1066 Xueyuan Ave, Shenzhen, Guangdong 17 Province, China, 518060; Phone: 86-755-26910912; Fax: 86-755-86671906; E-mail: 18 txiao@szu.edu.cn; Or Duo Zheng, Phone: 86-755-86674681; Fax: 86-755-86671906; 19 E-mail: dzheng@szu.edu.cn 


\section{Abstract}

2 Oncogenic KRAS mutations are considered to be a key driver for initiation and

3 progression in non-small-cell lung cancer (NSCLC). However, how post-translational

4 modifications (PTMs) of KRAS, especially methylation, modify KRAS activity and

5 downstream signals remain largely unclear. Here, we showed that SET domain

6 containing histone lysine methyltransferase 7 (SETD7) interacts with KRAS and

7 methylates KRAS at lysine 182 and 184. SETD7-mediated methylation of KRAS led

8 to degradation of KRAS and attenuation of the RAS/MEK/ERK signaling cascade,

9 endowing SETD7 with a potent tumor-suppressive role in NSCLC, both in vitro and in

10 vivo. Mechanistically, RABGEF1, a ubiquitin E3 ligase of KRAS, was recruited and

11 promoted KRAS degradation in a K182/K184 methylation-dependent manner. Notably,

12 low SETD7 or RABGEF1 expression was associated with poor prognosis in lung

13 adenocarcinoma patients. Taken together, our results establish a novel connection

14 between lysine methylation and KRAS protein stability, in addition to elucidating a

15 tumor-suppressive function of SETD7 that operates via modulation of oncogenic RAS

16 signaling. 


\section{Introduction}

2 More than $30 \%$ of all human cancers are driven by mutations in RAS

3 proto-oncogenes (Prior et al., 2012), which comprise four distinct isoforms: HRAS,

4 NRAS, and two splice variants of the KRAS gene, KRAS-4A and KRAS-4B (Hobbs et

5 al., 2016). The mutation frequencies of RAS isoforms differ in distinct cancer types. In

6 pancreatic ductal adenocarcinoma (PDAC) and lung adenocarcinoma (LUAD), KRAS

7 accounts for almost $100 \%$ of all RAS mutations (Cox et al., 2014). Oncogenic RAS

8 mutations usually occur at the conserved residues G12, G13, or Q61 (Brose et al.,

9 2002), which favor an active GTP-bound state and produce sustained activation of

10 downstream signaling (Scheffzek et al., 1997; Trahey and McCormick, 1987). For their

11 biological activity, RAS proteins must be anchored to the plasma membrane (PM)

12 (Zhou et al., 2017), where they recruit a variety of effectors to transduce signals from

13 tyrosine kinase receptors and stimulate signaling cascades (Simanshu et al., 2017).

14 Protein post-translational modifications (PTMs) are critical for translocation of

15 RAS to the PM (Ahearn et al., 2018). Primary translation products of RAS undergo

16 CAAX processing following a second signal; this signal comprises

17 mono-palmitoylation on Cys residues located upstream of the CAAX motif for NRAS

18 and KRAS-4A and duo-palmitoylation for HRAS (Hancock et al., 1989). KRAS-4B is

19 not modified by palmitic acid (Hancock et al., 1989). Instead, it has a polybasic 
1 domain with eight lysines that are strongly positively charged and allow for

2 electrostatic interactions with head groups of PM lipids (Hancock et al., 1990).

3 Although KRAS-4B requires no second signal beyond CAAX processing to associate

4 with membranes, the affinity of the interaction is still regulated by PTM.

5 Phosphorylation of serine 181 (S181) by protein kinase C (PKC) within the polybasic

6 region can partially neutralize the positive charge and mediate translocation of

7 KRAS-4B from the PM to the endomembrane system (Bivona et al., 2006). S181

8 phosphorylation is essential for oncogenic KRAS function in activation of p-AKT and

9 p-ERK1/2, as well as promoting cell proliferation, mobility, and tumor growth

10 (Alvarez-Moya et al., 2010; Barcelo et al., 2014b). However, other types of conditional

11 PTMs, especially methylation, and their role in modifying KRAS-4B localization and

12 activity remain largely unknown.

13 Protein lysine methyltransferases (PKMTs) have been shown to methylate

14 histone and non-histone proteins and participate in the regulation of several biological

15 processes in both healthy human physiology and human diseases (Copeland et al.,

16 2009). SET domain containing histone lysine methyltransferase 7 (SETD7), also

17 known as SET7/9, KIAA1717, or KMT7, was first identified as a

18 mono-methyltransferase of lysine 4 on histone H3 (H3K4) (Wang et al., 2001). The

19 lysine residue methylated by SETD7 frequently followed the consensus amino acid 
1 motif [K/R]-[S/T/A](Del Rizzo and Trievel, 2011). Recently, several fundamental

2 proteins involved in tumor progression have been identified as non-histone substrates

3 of SETD7, including the tumor suppressor p53 (Campaner et al., 2011; Chuikov et al.,

4 2004; Kurash et al., 2008), NF-kB p65 (Yang et al., 2009), hypoxia-induced factor-1 $\alpha$

5 (HIF-1 $\alpha)($ Kim et al., 2016), $\beta$-catenin (Shen et al., 2015), and nuclear hormone

6 estrogen receptor alpha $(\mathrm{ER} \alpha)$ (Subramanian et al., 2008). The fates of proteins

7 modified by SETD7-mediated lysine methylation are quite diverse. While methylation

8 of $\mathrm{p} 53$ or ER $\alpha$ stabilized these proteins and enhanced transcriptional activity,

9 methylation of $\beta$-catenin at K180 by SETD7 promoted ubiquitination and proteasomal

10 degradation (Shen et al., 2015). Methylation at different lysines within a protein can

11 even lead to divergent outcomes. For instance, SETD7 methylates the nuclear NF-kB

12 p65 subunit at $\mathrm{K} 37$, which restricts p65 to the nucleus and facilitates its binding to

13 promoters of inflammatory genes (Ea and Baltimore, 2009), whereas methylation at

14 K314 and K315 destabilizes p65 by promoting ubiquitination (Yang et al., 2009).

15 SETD7 can positively or negatively regulate multiple proteins; therefore, the function

16 of SETD7 in tumor progression may be dependent on the cellular context and the

17 substrate (Batista and Helguero, 2018). SETD7 contains three membrane occupation

18 and recognition nexus (MORN) motifs in the N-terminal region, which is believed to

19 mediate SETD7's interaction with and anchorage to the PM (Bivona et al., 2006). 
1 However, to date, no proteins located in the PM have been identified as substrates of

2 SETD7.

3 Here, by quantitative proteomics based on spectra count method, we

4 determined that SETD7 interacts with KRAS on the PM and methylates KRAS at

5 lysines 182 and 184 . These PTMs promoted KRAS protein degradation by recruiting

6 the E3 ligase, RabGEF1. Thus, SETD7 plays a potent tumor-suppressive role in

7 KRAS-mutated NSCLC via the downregulation of RAS signaling. SETD7 was also

8 downregulated in clinical lung cancer specimens and low SETD7 expression appeared

9 to be associated with poor prognosis. Of note, SETD7 expression was inversely

10 correlated with KRAS protein levels but not mRNA levels, further supporting the role

11 played by SETD7 in PTMs of KRAS. 


\section{$1 \quad$ Results}

\section{SETD7 interacts with KRAS but not HRAS or NRAS}

3 To identify the potential post-translational modifications of KRAS-4B (hereafter

4 referred to as KRAS), we first explored KRAS interactome in the human embryonic

5 kidney 293 T cells via the ectopic expression of $3 \times$ HA-tagged KRAS (HA-KRAS). We

6 prepared cellular extracts and incubated them with anti-HA magnetic beads. After

7 extensive washing, bound proteins were eluted with a basic buffer and resolved by

8 SDS-PAGE (Figure 1A), and then followed by liquid chromatography tandem mass

9 spectrometry (LC-MS/MS) analysis (Figure 1B). As expected, Ras GTPase-activating

10 protein-binding protein 1 and 2 (G3BP1/G3BP2), as well as Ras-related proteins

$11 \mathrm{RAB} 11 \mathrm{~A} / \mathrm{RAB} 7 \mathrm{~A} / \mathrm{RAB} 6 \mathrm{~A}$ were detected as candidate KRAS-interacting proteins

12 (Supplementary Table 1). Consistent with previous report, ribonucleoprotein

13 HNRNPA2B1 which interacted with and regulated oncogenic KRAS in PDAC

14 cells(Barcelo et al., 2014a) was also found in the KRAS interactome. Of note, by

15 quantitative proteomics based on spectra count method, SETD7 was identified as the

16 most significantly differential protein enriched in the immunoprecipitation (IP) sample

17 of HA-KRAS-expression cells compared to that of vector control (Figure 1C,

18 Supplementary Figure S1A). Intriguedly, SETD7 only interacted with KRAS and not

19 the other two RAS family members, HRAS and NRAS (Figure 1D). Confocal 
1 microscopy analysis showed that SETD7 was co-localized with KRAS, predominantly

2 on the plasma membrane, where KRAS recruits regulators and effectors to exert its

3 function (Figure 1E). The interaction between KRAS and SETD7 was further

4 confirmed by co-immunoprecipitation (co-IP) in both directions in the NSCLC cell line

5 NCI-H358 (H358) with stable SETD7 overexpression (Figure 1F and G). Endogenous

6 KRAS protein was also immunoprecipitated from A549 cell extracts using anti-SETD7

7 antibody, indicating an interaction between KRAS and SETD7 at the physiological

8 level (Figure 1H).

9 SETD7 is a potential tumor suppressor in lung cancer cells with KRAS mutations

10 To understand the role of SETD7 in NSCLC progression, we first checked the

11 expression of SETD7 in NSCLC cell lines and clinical specimens. According to

12 staging information relating to NSCLC cell lines, obtained from the American Type

13 Culture Collection (ATCC), we found that SETD7 expression was much lower in cell

14 lines derived from late-stage tumors (Supplementary Figure S1B).

15 Since SETD7 exclusively interacts with KRAS, and not HRAS and NRAS, we

16 next examined the potential function of SETD7 in NSCLC cell lines harboring KRAS

17 mutations. In H358 (KRAS G12C) and A549 (KRAS G12S) cells, overexpression (OE)

18 of SETD7 impaired both cell proliferation and two-dimensional (2-D) colony

19 formation (Figure 2A and B, Supplementary Figure S2A and B). The spheroid 
1 growth of $\mathrm{H} 358$ cells in soft-agar culture was also attenuated with ectopic SETD7 OE

2 (Figure 2C), implying a tumor-suppressive effect of SETD7 in NSCLC. To test

3 whether the anti-tumor effect of SETD7 was associated with its interaction with KRAS,

4 we examined the impact of SETD7 on KRAS and downstream signaling cascades.

5 Interestingly, along with downregulation of KRAS at the protein level, two critical

6 downstream effectors, phospho-ERK1/2 (p-ERK1/2) and phospho-AKT (p-AKT), also

7 apparently decreased when SETD7 was overexpressed in H358 (Figure 2D) and A549

8 cells (Supplementary Figure S2C). On the other hand, efficient silencing of SETD7

9 by short hairpin RNAs (shRNAs) strongly promoted cell growth and colonization

10 (Figure 2E and F, Supplementary Figure S2D and E), in addition to enhancing

11 anchorage-independent cell growth in NCI-H1437 (H1437) (Figure 2G,

12 Supplementary Figure S2F) and H358 cells (Supplementary Figure S2G). As

13 expected, knockdown (KD) of SETD7 abrogated its inhibition of KRAS expression,

14 leading to elevated levels of KRAS protein and the reactivation of p-ERK1/2 and

15 p-AKT (Figure 2H and Supplementary Figure S2H).

16 We further investigated the anti-tumor effect of SETD7 in vivo. A549

17 cells with stable expression of SETD7 or a vector control were subcutaneously injected

18 into the opposite flanks of nude mice. The growth rate of xenograft tumors with

19 SETD7 OE was significantly attenuated (Figure 3A). Compared with the results of 
1 paired control tumors, both the volume and weight of tumors with SETD7 OE were

2 lower at 7 weeks post-injection (Figure 3B, Supplementary Figure S3A).

3 Immunohistochemical staining of tumor tissues revealed that SETD7 downregulated

4 endogenous KRAS expression and inhibited tumor cell proliferation, which was

5 characterized by a decreased percentage of Ki67-positive cells (Figure 3C). For H1437

6 cells with relatively high SETD7 expression (Supplementary Figure S1B), their

7 tumorigenic ability in nude mice seemed poor, as growth of the subcutaneous

8 xenograft was very slow and even ceased one week post-injection (Figure 3D).

9 Notably, SETD7 knockdown in H1437 cells greatly accelerated xenograft tumor

10 growth (Figure 3D) and consequently boosted both the volume and weight of tumors

11 with SETD7 KD at 4 weeks post-injection (Figure 3E, Supplementary Figure S3B).

12 As a consequence of SETD7 knockdown, both the KRAS level and percentage of

13 Ki67-positive cells were simultaneously elevated in xenograft tumor tissues (Figure

14 3F). Taken together, these in vitro and in vivo findings indicate that SETD7 suppressed

15 NSCLC progression via the negative regulation of KRAS expression and downstream

$16 \mathrm{MEK} / \mathrm{ERK}$ and PI3K/AKT signaling pathways.

\section{SETD7 promotes KRAS ubiquitination and degradation}

18 To elucidate the molecular mechanism underlying SETD7-mediated KRAS

19 downregulation, we first sought to determine whether SETD7 impinges on KRAS 
1 transcription. Quantitative PCR results showed that neither SETD7 OE in A549 and

2 H358 cells (Supplementary Figure S4A and B) nor knockdown in H1437 cells

3 changed KRAS mRNA expression (Supplementary Figure S4C). Thus, we

4 speculated that the interaction of SETD7 with KRAS may influence KRAS protein

5 stability. To test this hypothesis, the protein levels of KRAS were determined at

6 different time points following treatment with cycloheximide (CHX), an inhibitor of

7 protein synthesis in eukaryotes. We found that co-expression of SETD7 and KRAS in

$8293 \mathrm{~T}$ cells accelerated the degradation of exogenous KRAS proteins (Figure 4A).

9 Additionally, SETD7 OE obviously stimulated the turnover rate of endogenous KRAS

10 in H358 and A549 cells (Figure 4B and Supplementary Figure S4D), whereas

11 knockdown of SETD7 prolonged the half-life of KRAS proteins (Figure 4C).

To determine whether SETD7 methyltransferase activity is indispensable for

13 KRAS degradation and ubiquitination, we constructed a catalytically dead SETD7 ${ }^{\mathrm{H} 297 \mathrm{~A}}$

14 mutant(Nishioka et al., 2002). As expected, OE of wild-type (WT) SETD7 markedly

15 enhanced degradation (Figure 4E) and ubiquitination of KRAS (Figure 4F). However,

16 the H297A mutant of SETD7, which lacks lysine methyltransferase activity(Nishioka

17 et al., 2002), could interact with KRAS (Figure 4D) but had lost the ability to promote

18 KRAS degradation (Figure 4E) and ubiquitination (Figure 4F), suggesting this

19 catalytic activity is critical for SETD7-mediated KRAS ubiquitination and degradation. 
1 The suppression of cell growth and 2-D colony formation by WT SETD7 in H358 and

2 A549 cells was reversed by the H297A mutant (Figure 4G and H, Supplementary

3 Figure S4E and F). SETD $7^{\mathrm{WT}}$-inhibited growth of three-dimensional (3-D) spheroids

4 in H358 and A549 cells was also restored by the H297A mutant (Figure 4I and

5 Supplementary Figure S4G). Accordingly, SETD $7^{\mathrm{WT}}$ repression of the KRAS-related

6 pathway was rescued by SETD $7^{\mathrm{H} 297 \mathrm{~A}}$ (Figure 4J and Supplementary Figure S4H).

7 These results imply that SETD7 reduces KRAS protein stability through ubiquitination,

8 in a catalytic-dependent manner.

9 SETD7 methylates KRAS at K182 and K184 and facilitates E3 ligase RabGEF1

10 recruitment

11 We determined that SETD7-mediated KRAS ubiquitination and degradation were

12 dependent on its methyltransferase activity, so we further investigated whether KRAS

13 is a direct substrate of SETD7. Among known SETD7 substrates, the methylated lysine

14 site usually follows the conserved amino acid motif [K/R]-[S/T/A](Del Rizzo and

15 Trievel, 2011). By analyzing the amino acid sequence of KRAS-4B, two lysine

16 residues close to the C-terminal, lysine 182 (K182) and lysine 184 (K184), were

17 identified as potential target methylation sites for SETD7 (Figure 5A). However, the

18 consensus motif was not found in KRAS-4A, NRAS, or HRAS (data not shown).

19 Using a computer simulation, the complex between SETD7 and KRAS could only be 
1 stabilized in trajectory from structure (str.) 4 of the simulated styles (Supplementary

2 Figure S5A and B). Based on the str. 4 model, K182 and K184, together with T183,

3 formed an interactional network with SETD7 and helped KRAS bind to SETD7

4 (Figure 5B). As predicted, KRAS methylation was enhanced by co-expression of WT

5 SETD7 but not the catalytically dead H297A mutant (Figure 5C). Mutation of K182 or

6 K184 to methionine obviously reduced the level of KRAS methylation compared with

7 that of the WT group (Figure 5D). Of note, the interaction between SETD7 and KRAS

8 was also attenuated following $\mathrm{K} 182$ and K184 mutation (Figure 5D). To further

9 confirm SETD7 methylates KRAS at K182 and K184, we performed

10 immunoprecipitation with anti-HA magnetic beads in 293T cells with co-expression of

11 SETD7 $^{\mathrm{WT}}$ (or SETD7 $7^{\mathrm{H} 297 \mathrm{~A}}$ ) and $3 \times \mathrm{HA}$-tagged KRAS, and identified the lysines

12 methylation on KRAS using LC-MS/MS analysis. K182 methylation of KRAS was

13 detected in SETD $7^{\mathrm{WT}}$ group but not in SETD $7^{\mathrm{H} 297 \mathrm{~A}}$ group, supporting our novel finding

14 that KRAS is a direct substrate of SETD7 (Figure 5E and Supplementary Figure

15 S5C).

16 We next sought to investigate the underlying mechanism via which methylation at

$17 \mathrm{~K} 182$ and K184 regulates KRAS protein stability. It has been reported that RabGEF1 is

18 an E3 ligase of the RAS family members HRAS and NRAS(Xu et al., 2010). Thus, we

19 asked whether RabGEF1 could act as an E3 ligase of KRAS. A co-IP assay showed an 
1 interaction between KRAS and RabGEF1 only in the presence of SETD7 ${ }^{\mathrm{WT}}$ but not in

2 SETD7 $^{\text {H297A }}$ group (Figure 5F). In addition, dual mutations of KRAS at K182 and

3 K184 (KRAS-DM) restricted its interaction with RabGEF1, suggesting that

4 methylation of KRAS by SETD7 facilitates the recruitment of RabGEF1 (Figure 5F).

5 While RabGEF1 alone could not promote KRAS degradation (Supplementary Figure

6 S5D), the co-expression of SETD7 and RabGEF1 worked synergistically to reduce the

7 half-lives of KRAS proteins (Supplementary Figure S5E). In contrast, KRAS-DM

8 exhibited greater protein stability, which was impervious to

9 SETD7/RabGEF1-mediated degradation (Figure 5G). Moreover, knockdown

10 endogenous RabGEF1 increased stability and extended the half-lives of KRAS

11 proteins (Supplementary Figure S5F). Consistent with previous study(Xu et al.,

12 2010), RabGEF1 alone couldn't induce KRAS ubiquitination (Figure 5H). However,

13 in accordance with the reduced half-life of KRAS, SETD7 and RabGEF1 cooperated to

14 increase the ubiquitination of WT KRAS, whereas KRAS-DM exhibited a

15 ubiquitination-resistant phenotype (Figure 5H). These results suggest that

16 RabGEF1-induced KRAS degradation and ubiquitination is dependent on

17 SETD7-mediated K182/184 methylation of KRAS protein.

18 Interestingly, in the presence of both SETD7 and RabGEF1, the protein stability

19 of the constitutively active KRAS G12D mutant also decreased (Figure 5G), 
1 accompanying with the increase of ubiquitination (Figure $\mathbf{5 H}$ ), implying that

2 SETD7/RabGEF1-mediated KRAS degradation may be widespread in mammalian

3 cells, regardless of KRAS mutation status. Compared with KRAS G12D activity,

4 KRAS G12D-DM showed a more obvious effect on promoting cell proliferation

5 (Figure 5I) and activating downstream MEK/ERK and PI3K/AKT signaling in H1437

6 cells (Figure 5J), suggesting that the prolonged half-life and increased protein stability

7 conferred a more potent function on KRAS. Taken together, these data indicate that

8 methylation of KRAS by SETD7 at the K182 and K184 residues promotes

9 ubiquitination and degradation through the recruitment of the E3 ligase RabGEF1.

10 SETD7 is downregulated in clinical NSCLC samples and inversely correlated with

11 KRAS at the protein level

12 Since SETD7 suppressed NSCLC progression by promoting KRAS methylation

13 and degradation, we further sought to determine the clinical significance of SETD7 in

14 NSCLC. Analysis of the Kaplan-Meier (KM) plotter database (Gyorffy et al., 2013)

15 revealed that increased SETD7 expression was associated with better prognosis in

16 patients with NSCLC or lung adenocarcinoma (LUAD) (Figure 6A), but not in

17 patients with lung squamous cell carcinoma (LUSC) (Supplementary Figure S6A).

18 Our database analysis also showed that RabGEF1 acted as a tumor suppressor in lung

19 cancer, especially LUAD (Supplementary Figure S6B). The combined SETD7 and 
1 RabGEF1 profiles showed that only patients with simultaneously increased expression

2 of SETD7 and RabGEF1 exhibited longer survival times (Supplementary Figure

3 S6C). In the population with increased SETD7 expression, decreased RabGEF1

4 expression was clearly associated with poor survival in LUAD, and vice versa

5 (Supplementary Figure S6D and E). More importantly, in the decreased

6 SETD7-expression (or RabGEF1) population, increased expression of RabGEF1 (or

7 SETD7) did not confer any survival advantage, implying that SETD7 and RabGEF1

8 might cooperate to execute their tumor-suppressive roles in LUAD progression

9 (Supplementary Figure S6F and G). In our own clinical cohort with NSCLC, the

10 expression of SETD7 varied among patients (Figure 6B) and those who possessed

11 high SETD7 expression also displayed better prognoses (Figure 6C). 


\section{Discussion}

2 Here, we describe for the first time that lysine residues on KRAS protein are

3 methylated by SETD7. SETD7 interacts with KRAS and methylates it at K182 and

4 K184. This methylation destabilizes KRAS via the enhancement of ubiquitin-mediated

5 protein degradation by the E3 ligase RabGEF1. Interestingly, the epigenetic codes

6 written by SETD7 appear critical for the recruitment of RabGEF1 to KRAS. KM

7 plotter analysis also showed SETD7 and RabGEF1 act synergistically to prolong

8 overall survival in patients with LUAD. These findings imply that SETD7 may

9 cooperate with RabGEF1 to exert an anti-cancer effect in NSCLC, and that the

10 combination of SETD7 and RabGEF1 may serve as a useful index for prognostic

11 prediction in KRAS-driven LUAD.

13 identity in their $\mathrm{N}$-terminals. The variation in the $\mathrm{C}$-terminal among the four isoforms

14 is critical for determining the subcellular localization of RAS (Simanshu et al., 2017).

15 Although phosphorylation of serine 181 (S181) on the C-terminal tail of KRAS-4B by

16 protein kinase $\mathrm{C}(\mathrm{PKC})$ has been well studied for its role in regulating KRAS

17 translocation and signaling transduction, the outcome of KRAS phosphorylation at

18 S181 remains controversial. Some studies have shown that S181 phosphorylation of

19 KRAS, which is inhibited by calmodulin $(\mathrm{CaM})$, is essential for oncogenic KRAS 
1 function in activation of $\mathrm{p}-\mathrm{AKT}$ and $\mathrm{p}-\mathrm{ERK} 1 / 2$, as well as promoting cell proliferation,

2 mobility, and tumor growth (Alvarez-Moya et al., 2010; Barcelo et al., 2014b).

3 However, others have reported that PKC-mediated S181 phosphorylation could either

4 induce KRAS-4B translocation from the $\mathrm{PM}$ to mitochondria and trigger

5 Bcl-XL-dependent apoptosis (Bivona et al., 2006), or impair the KRAS-CaM

6 interaction and activate non-canonical Wnt signaling, which prevents tumorigenicity

7 (Wang et al., 2015). Specifically, phosphorylation of GTP-bound KRAS-4B on S181

8 was shown to reduce nanocluster formation (Plowman et al., 2008), a spatial

9 organization of RAS upon which signal transmission is dependent (Tian et al., 2007). A

10 negatively charged phosphate group on S181 would repel cell membrane phospholipids,

11 trigger the dissociation of KRAS from the cell membrane, and transport KRAS back to

12 subcellular compartments (Feig, 2006); therefore, it is most likely that S181

13 phosphorylation negatively regulates KRAS function. Thus, PKC agonists or activators

14 have been proposed for use as anti-cancer agents to inhibit oncogenic, KRAS-driven

15 malignancies (Bivona et al., 2006; Wang et al., 2015). In our study, we found that

16 SETD7 overexpression also induced translocation of KRAS from the PM to the cytosol,

17 similar to the effect of S181 phosphorylation, whereas K182/K184 mutations could

18 reverse this phenotype (unpublished data). Since methylation does not change the

19 positive charge of lysine residues, we speculated that K182/K184 methylation might be 
1 a prerequisite for S181 phosphorylation of KRAS. We further noticed that K182/184M

2 or S181A mutations of KRAS were resistant to ubiquitination-mediated degradation

3 (Figure $6 \mathrm{H}$ and unpublished data), suggesting that PTM of residues located in

4 polybasic domains links the subcellular localization of KRAS to its protein stability.

5 Thus, further elucidation of the interplay between K182/K184 methylation and S181

6 phosphorylation will be important for understanding how different types of PTM

7 cooperate to modulate KRAS protein stability.

8 SETD7 is an important modifier of several non-histone proteins and exerts either

9 oncogenic or tumor-suppressive roles in different types of cancer, depending on the

10 cellular context (Batista and Helguero, 2018). Previous studies have shown that

11 SETD7 serves as a tumor suppressor in breast cancer (Montenegro et al., 2016), renal

12 cellular carcinoma (Liu et al., 2015), colorectal cancer, and gastric cancers(Hong et al.,

13 2018). Nevertheless, an oncogenic role for SETD7 has also been reported in breast

14 cancer (Subramanian et al., 2008), prostate cancer (Wang et al., 2018), hepatocellular

15 carcinoma (Chen et al., 2016), and intestinal tumorigenesis (Oudhoff et al., 2016), via

16 various molecular mechanisms. The controversial role SETD7 plays in different types

17 of cancer suggests that the function of SETD7 in cancer progression depends on the

18 cellular or tissue context and specific interacting proteins. We found that SETD7

19 inhibited KRAS-driven LUAD both in vitro and in vivo. Surprisingly, in NSCLC cell 
1 lines with constitutively active EGFR mutations, SETD7 overexpression promoted cell

2 growth (unpublished data). Since EGFR mutant cell lines were not sensitive to

3 inhibition of MEK (Tricker et al., 2015), a direct downstream effector of RAS, SETD7

4 may interact with other, undefined substrates and play an oncogenic role in an

5 EGFR-mutated genetic context. To date, KRAS-driven lung cancer remains an

6 intractable disease. In view of the promising anti-cancer effect of SETD7 in NSCLC

7 cell lines with KRAS addiction, approaches involving the upregulation of SETD7

8 expression or activation of SETD7 enzymatic activity might benefit lung cancer

9 patients with KRAS mutations. Furthermore, since KRAS and EGFR mutations are

10 usually mutually exclusive in LUAD (Ding et al., 2008), further exploration of the

11 distinct roles of SEDT7 in KRAS- or EGFR-mutant contexts, which are the most

12 critical oncogenic drivers in LUAD, will be helpful in the development of novel

13 strategies for targeted therapies in lung cancer.

14 In summary, we have demonstrated that SETD7 inhibits NSCLC progression by

15 downregulating KRAS signaling cascades. SETD7 can methylate KRAS at K182 and

$16 \mathrm{~K} 184$, further enhancing KRAS ubiquitination and degradation via the recruitment of

17 the E3 ligase, RabGEF1 (Figure 7). Our data suggest that the combination of SETD7

18 and RabGEF1 will be a useful index for prognostic prediction in NSCLC cases. 


\section{Materials and Methods}

\section{Human Lung Cancer Specimen Analysis}

3 Tissue Microarray (TMA) for survival analysis was obtained from 127 patients with

4 lung cancer who underwent potentially curative pulmonary resection at

5 the Department of Thoracic Surgery, the Second Xiangya Hospital of Central South

6 University from 2002-2012. Patients enrolled in this study were based on pathologic

7 diagnosis of lung adenocarcinoma, each sample containing a minimum of $70 \%$ tumor

8 cells as determined by study pathologists. Approval was given in advance by our

9 institutional review board, and all patients gave written informed consent. These

10 patients had been subjected to routine staging and definitive surgical resection of the

11 lung and systematic mediastinal lymph node dissection. No patients had been

12 previously treated with chemotherapy and radiotherapy at the time of original

13 operation. Complete clinical record and follow-up data were available for all patients.

14 Overall survival time was calculated from the data of diagnosis to the date of death or

15 the data last known alive.

16 To examine the SETD7 expression profile, the TMA sections were labeled with

17 an SETD7 antibody (Abcam, 1:200). Immunohistochemical (IHC) staining of TMA

18 sections were scored independently by qualified pathologists blinded to the

19 clinicopathological data, at $\times 200$ magnification light microscopy. Staining intensity 
1 was classified as 0 (negative), 1 (weak), 2 (moderate) or 3 (strong). According to these

2 scores, an optimal cut-off point was identified as follows: a staining index score of 0-1

3 was used to define tumors with low expression and 2-3 indicated high expression of

4 SETD7 protein. Agreement between the evaluators was 95\%, and all scoring

5 discrepancies were resolved through discussion between the evaluators. Kaplan-Meier

6 analysis was performed for overall survival.

$7 \quad$ Reagents, enzymes, and antibodies

8 Chemical reagents and reaction buffers were obtained from Sigma-Aldrich (St. Louis,

$9 \mathrm{MO}, \mathrm{USA}) . \mathrm{Q} 5^{\circledR}$ High-Fidelity DNA Polymerase, DNA ligase and the restriction

10 enzymes were purchased from New England BioLabs (NEB, Ipswich, MA, USA). A

11 list of antibodies used is provided in the Supplementary Materials.

\section{Construction of plasmids and mutagenesis}

13 Full-length human SETD7 cDNA sequences were amplified from a human mRNA

14 pool generated by RT-PCR using SuperScript II RNase H Reverse Transcriptase (Life

15 Technologies,CA, USA). The SETD7 cDNA was cloned into the EcoRI/BamHI site of

16 a pCDH-CMV-MCS-EF1 $\alpha$-Puro vector (System Biosciences, Palo Alto, CA, USA ).

17 The sequences were confirmed by Sanger sequencing. Point mutations were performed

18 using Q5 DNA polymerase to generate KRAS K182/184M mutants. The primary DNA

19 template was digested with DpnI for 2 hours at $37^{\circ} \mathrm{C}$, then the mutant pCDH-KRAS 
1 was transformed and amplified by DH5 $\alpha$ competent cells. The primers used for

2 cloning and mutagenesis are provided in the Supplementary Materials.

\section{Cell culture and stable pools selection}

4 HEK293T cells were obtained from Cell Bank of Chinese Academy of Sciences and

5 cultured in Dulbecco's modified Eagle's medium (DMEM; Life Technologies) with

$610 \%$ fetal bovine serum (FBS; PAN Seratech, Aidenbach, Germany) and $100 \mathrm{U} / \mathrm{ml}$

7 penicillin-streptomycin (Life Technologies). The human non-small-cell lung cancer

8 (NSCLC) cell lines A549, NCI-H358, NCI-H1437, and NCI-H522 were obtained from

9 the American Type Culture Collection (ATCC) and cultured in RPMI1640 (Life

10 Technologies) containing $10 \%$ FBS (PAN Seratech) and $100 \mathrm{U} / \mathrm{mL}$

11 penicillin-streptomycin (Life Technologies). HEK293T, A549, NCI-H358, NCI-H1437,

12 NCI-H522, and stable pools were incubated at $37^{\circ} \mathrm{C}$ with $5 \% \mathrm{CO}_{2}$. All cell lines were

13 authenticated by short tandem repeat (STR) profiling (Guangdong Huaxi Forensic

14 Physical Evidence Judicial Appraisal Institute, Guangdong, China). The cell lines were

15 passaged fewer than ten times or 6 months after initial revival from frozen stocks; they

16 tested negative for mycoplasma.

17 For generation of stable pools with SETD7 overexpression or knockdown,

18 NSCLC cell lines were infected by lentivirus expression of SETD7 or SETD7 shRNA

19 and selected by culturing in RPMI1640 complete medium containing $10 \mu \mathrm{g} / \mathrm{mL}$ 
1 puromycin (Selleck, TX, USA) for 1 week. SETD7 expression was evaluated by qPCR

2 and western blotting. Functional assays were performed after confirmation of gene

3 expression. The shRNA oligonucleotides used are listed in the Supplementary

4 Materials.

\section{Real-time PCR}

6 Total RNA was extracted using Trizol reagent (Life Technologies) and cDNA was

7 synthesized using RevertAid First Strand cDNA Synthesis Kit (Thermo Scientific,

8 Waltham, MA, USA) according to the manufacturer's instructions. All real-time PCR

9 reactions were performed using the CFX Connect Real-Time PCR Detection System

10 (Bio-Rad, Hercules, CA, USA) and the amplifications were carried out using the

11 ChamQ Universal SYBR qPCR Master Mix (Vazyme, Nanjing, China). Each real-time

12 PCR reaction was repeated three times, and target genes were normalized using

13 GAPDH as an internal reference. Primers for real-time PCR were listed in

14 Supplementary Materials.

\section{Immunoprecipitation and western blot}

16 For immunoprecipitation, HEK293T cells transfected with indicated plasmids were

17 cultured in a 100-mm dish. Cells were collected using IP lysis buffer when they were

$1880 \%$ confluent. Following clarification, the supernatant fractions were used for

19 immunoprecipitation with anti-HA, anti-Flag, or anti-myc antibody and rotated at 5 
$1 \mathrm{rpm}$ at $4^{\circ} \mathrm{C}$ overnight. Next, protein $\mathrm{A} / \mathrm{G}$ magnetic beads (MedChemExpress,

2 Monmouth Junction, NJ, USA) was added and incubated for $4 \mathrm{~h}$. After washing the

3 magnetic beads several times with binding buffer (25 mM Tris- $\mathrm{HCl}, \mathrm{pH}$ 7.5,100 mM

$4 \mathrm{NaCl}, 0.1 \% \mathrm{NP}-40$ ), the immunoprecipitant was finally eluted with sample buffer

5 containing $1 \%$ SDS.

6 Western blotting was performed following immunoprecipitation. The protein

7 concentration was determined using a BCA Protein Assay Kit (Beyotime

8 Biotechnology, Shanghai, China). All protein samples were separated by sodium

9 dodecyl sulfate-polyacrylamide gel electrophoresis (SDS-PAGE), transferred to

10 polyvinylidene fluoride (PVDF) membranes (Merck Millipore, Burlington, MA, USA

11 ), hybridized with the corresponding antibodies, and detected using Pierce ECL

12 Western blotting substrate (Thermo Scientific).

\section{Immunofluorescence}

14 The transfected cells were grown on cover slide and fixed by $50 \%$ methanol for 10 min

15 at $4^{\circ} \mathrm{C}$. After incubation with anti-SETD7 or anti-KRAS antibodies (1:100) in PBS

16 containing $5 \%$ bovine serum albumin over night at $4^{\circ} \mathrm{C}$, samples were rinsed with $\mathrm{PBS}$,

17 incubated with Alexa Fluor 488 conjugated anti-mouse or Alexa Fluor 546 conjugated

18 anti-rabbit secondary antibodies (1:300, Life Technologies) in PBS containing 5\%

19 bovine serum albumin for 2 hours at room temperature avoiding light. After washing in 
1 PBS, samples were mounted by ProLong ${ }^{\text {TM }}$ Gold Antifade Mountant with DAPI (Life

2 Technologies) for observation. Images were acquired using a confocal microscopy

3 (LSM880, Carl Zeiss, Jena, Germany).

$4 \quad$ Mass spectrometry (MS) analysis of KRAS-interacting proteins

5 The beads samples obtained from immunoprecipitation experiment were washed three

6 times with pre-cooled PBS buffer to remove the remaining detergent. Then beads

7 samples were incubated in the reaction buffer (1\% SDC/100 mM Tris-HCl, pH 8.5/10

$8 \mathrm{mM} \mathrm{TCEP} / 40 \mathrm{mM} \mathrm{CAA}$ ) at $95{ }^{\circ} \mathrm{C}$ for $10 \mathrm{~min}$ for protein denaturation, cysteine

9 reduction and alkylation. The eluates were diluted with equal volume of $\mathrm{H}_{2} \mathrm{O}$ and

10 subjected to trypsin digestion overnight by adding $1 \mu \mathrm{g}$ of trypsin at $37^{\circ} \mathrm{C}$. The peptide

11 was purified using self-made SDB desalting columns. The eluate was vacuum dried

12 and stored at $-20^{\circ} \mathrm{C}$ for later use.

13 Mass spectrometry was performed using TripleTOF 5600+ LC-MS/MS system

14 (SCIEX). The peptide sample was inhaled by an autosampler and bound to a C18

15 capture column $(5 \mu \mathrm{m}, 5 \times 0.3 \mathrm{~mm})$, followed by elution to the analytical column $(300$

$16 \mu \mathrm{m} \times 150 \mathrm{~mm}, 3 \mu \mathrm{m}$ particle size, $120 \AA$ pore size, Eksigent) to carry out the

17 separation. Two mobile phases (mobile phase A: 3\% DMSO, 97\% H2O, 0.1\% formic

18 acid and mobile phase B: $3 \%$ DMSO, 97\% $\mathrm{ACN}, 0.1 \%$ formic acid) were used to

19 establish the analytical gradient. The flow rate of the liquid phase was set to $5 \mu \mathrm{L} / \mathrm{min}$. 
1 For mass spectrometry IDA mode analysis, each scan cycle contains a full MS scan

2 (m/z range 350-1250, ion accumulation time 250ms), followed by $40 \mathrm{MS} / \mathrm{MS}$ scans

$3(\mathrm{~m} / \mathrm{z}$ range is $100-1500$, ion accumulation time $50 \mathrm{~ms})$. The conditions for MS/MS

4 acquisition are set to a parent ion signal greater than $120 \mathrm{cps}$ and a charge number of

$5+2$ to +5 . The exclusion time for ion repeat acquisition is set to $18 \mathrm{~s}$.

6 Raw data from TripleTOF 5600 were analyzed with ProteinPilot (V4.5) using the

7 Paragon database search algorithm and the integrated false discovery rate (FDR)

8 analysis function. Spectra files were searched against the UniProt Human reference

9 proteome database using the following parameters: Sample Type, Identification; Cys

10 Alkylation, Iodoacetamide; Digestion, Trypsin; Search Effort was set to Rapid ID.

11 Search results were filtered with unused $\geq 1.3$. Decoy hits and contaminant proteins

12 were removed; the remaining identifications were used for further analysis.

\section{MS analysis of KRAS PTMs}

14 The bands corresponding to the KRAS proteins were cut and subjected to in-gel

15 digestion. Briefly, gels were destained with $50 \% 50 \mathrm{mM} \mathrm{NH}_{4} \mathrm{HCO}_{3} / 50 \% \mathrm{ACN}$, were

16 dehydrated with $100 \%$ ACN. After reduction and alkylation, proteins were digested

17 with addition of trypsin or chymotrypsin at $37 \square$ overnight. Peptides were dissolved in 
1 chromatography was performed on a reversed-phase column (40 $\mathrm{cm} \times 75$ um i.d.) at 50

$2{ }^{\circ} \mathrm{C}$ packed with Magic C18 AQ 3- $\mu \mathrm{m} 200-\AA$ resin with a pulled emitter tip. A solution

3 is $0.1 \% \mathrm{FA}$ in $\mathrm{H} 2 \mathrm{O}$, and $\mathrm{B}$ solution is $0.1 \% \mathrm{FA}$ in $\mathrm{ACN}$. In 120-min experiments,

4 peptides were separated with a linear gradient from 0\%-5\% B within 5min, followed

5 by an increase to $30 \% \mathrm{~B}$ within $105 \mathrm{~min}$ and further to $35 \% \mathrm{~B}$ within $5 \mathrm{~min}$, followed by

6 a washing step at $95 \% \mathrm{~B}$ and re-equilibration. The timsTOF pro was operated in

7 PASEF mode. The ddaPASEF files were searched against Swissprot human

8 (downloaded in September, 2018) appendant with common contaminants using Peaks

9 software. The search parameters were set as followed: parent monoisotopic tolerance

1015 ppm, product ion tolerance 0.05 Da, modification Carbamidomethyl $(\mathrm{C})$, potential

11 modification protein N-terminal acetylation, methylation on $\mathrm{KR}$, oxidation on $\mathrm{M}$ and

12 phosphorylation on STY, and maximum missed cleavage sites 2. The MS/MS spectra

13 of identified modified peptides were validated manually.

\section{Cell growth and 2-D clonogenic assay}

15 For cell growth assay, cells were seeded in 96-well plates at concentrations of 1-2 $\times$

$1610^{3}$ cells per well. Cells were incubated at $37^{\circ} \mathrm{C}$ in a final volume of $100 \mu \mathrm{L}$ culture

17 medium per well. Cell viability was evaluated at $0,24,48$, and $72 \square$ hours after cell

18 attachment. Cell Counting Kit-8 (CCK-8, Meliun Biotechnology, China) reagent (10

$19 \mu \mathrm{L}$ ) was added to each well, and the plates were incubated for 2 hours at $37^{\circ} \mathrm{C}$. The 
1 absorbance was measured at $450 \mathrm{~nm}$.

2 For 2-D clonogenic assay, cells were then seeded in 6-well plates at a

3 concentration of 500 cells per well. The cells were incubated at $37^{\circ} \mathrm{C}$ in a final volume

4 of $4 \mathrm{~mL}$ culture medium per well. After incubation for 8 to 10 days, the 6-well plates

5 were washed with $1 \times$ PBS and the cells were fixed by adding $100 \%$ methanol and

6 incubating them at $4{ }^{\circ} \mathrm{C}$ for 10 minutes. The cells were then stained with $0.5 \%$ crystal

7 violet for 10 minutes at room temperature before being washed twice in

8 double-distilled (dd) $\mathrm{H}_{2} \mathrm{O}$.

\section{Soft agar assay and 3-D spheroid formation assay}

10 For soft agar assay, cells were seeded in 0.4\% low-melting agarose (Sigma-Aldrich) in

11 6-well plate at a concentration of $5 \times 10^{3}$ cells per well. The cells were incubated at

$1237^{\circ} \mathrm{C}$ for $2-3$ weeks, then stained with $0.005 \%$ crystal violet for 1 hour at room

13 temperature before being destained in $\mathrm{ddH}_{2} \mathrm{O}$.

14 In spheroid formation assay, cells were seeded in 96-well

15 microplate (U-bottom) with cell-repellent surface (Greiner Bio-One GmbH,

16 Frickenhausen, Germany) at a concentration of $2 \times 10^{3}$ cells per well. Cells were

17 incubated at $37^{\circ} \mathrm{C}$ in a final volume of $200 \mu \mathrm{L}$ culture medium per well for 2 weeks.

18 The volume of spheroids were monitored by inverted microscopy and calculated by

19 use of the modified ellipsoid formula $1 / 2\left(\right.$ length $\times$ width $^{2}$ ). 


\section{Computer simulation of the molecular structure}

2 For the computer simulation, SETD7 and KRAS crystal structures were

3 obtained from the Protein Data Bank (PDB)(Berman et al., 2000). The G117-K366

4 sequence of SETD7 (PDB ID: 1O9S)(Xiao et al., 2003) and the M1-C185 sequence

5 (PDB ID: 6CCX)(Fang et al., 2018) of KRAS, both determined by X-ray diffraction,

6 were selected as the initial docking structures. The HADDOCK server (version 2.4,

7 http://milou.science.uu.nl/services/HADDOCK2.2/), set to default parameter values,

8 was used to perform simulations of KRAS docking with the SET7 binding pocket. The

9 most common structures from clusters 1, 2, 3, and 5 were fed into the dynamic

10 molecular simulation. In this study, all simulations were performed using the AMBER

11 package. The FF14SB force field was applied for the protein preparation simulation.

12 The complex was immersed in TIP3PBOX. Counter ions were added to neutralize the

13 system. First, the entire system was minimized until convergence occurred. After

14 minimization, the system, with restraint on all heavy atoms, was gradually heated up

15 to $300 \mathrm{~K}$, in NVT ensemble. The force constant of restraint was set to 100

$16 \mathrm{kcal} /(\mathrm{mol} \cdot \mathrm{rad} 2)$. Then, the system was relaxed in NPT ensemble in four stages, where

17 the force constant was set to $50,20,10$, and $5 \mathrm{kcal} /(\mathrm{mol} \cdot \mathrm{rad} 2)$, respectively. The entire

18 simulation lasted for $100 \mathrm{~ns}$, with a snapshot taken every ps. Cluster analysis was

19 performed on the last 20-ns trajectory. The linkage method was selected and the cutoff 
1 was set to $4.0 \AA$.

\section{Xenografts in nude mice}

3 All animal research procedures were performed according to the detailed rules of the

4 Animal Care and Use Ethics Committee of Shenzhen University Health Science Center,

5 and all animals were treated in strict accordance with protocols approved by the

6 Institutional Animal Use Committee of the Health Science Center, Shenzhen

7 University. Male $n u / n u$ nude mice, 4 to 6 weeks old, were subcutaneously injected in

8 their back with $5 \times 10^{6}$ stable pool cells containing A549-pCDH-puro,

9 A549-pCDH-SETD7, H1437-scramble control, and H1437-pLKO.1-shSETD7\#1 or

10 H1437-pLKO.1-shSETD7\#2. Tumor volume was monitored once per week. Tumor

11 volume was calculated according to the formula: volume $=$ length $\times$ width $^{2} / 2$. After 4

12 to 7 weeks, mice were euthanized, and their tumor xenografts were harvested for

13 weighing and following IHC staining for SETD7, KRAS, and Ki-67.

\section{$14 \quad$ Statistical analyses}

15 All in vitro experiments were performed at least in triplicate. The results of each

16 experiment are presented as the mean \pm standard deviation. Data analyses were

17 performed using Microsoft Excel 2010 Professional Plus (Version 14.0.7237.5000) and

18 Prism 6 (Version 6.01). Two-tailed, unpaired Student's t-tests were used to compare 
bioRxiv preprint doi: https://doi.org/10.1101/2021.02.10.429309; this version posted February 10, 2021. The copyright holder for this preprint (which was not certified by peer review) is the author/funder, who has granted bioRxiv a license to display the preprint in perpetuity. It is made available under aCC-BY-NC-ND 4.0 International license.

1 differences between two groups with similar variances. For all tests, a p-value $<0.05$

2 was considered to indicate a statistically significant difference.

3 


\section{Acknowledgments}

2 This study was supported by grants from the National Natural Science Foundation of

3 China (81974366, 81773019), the Natural Science Foundation of Guangdong Province

4 (2019A1515010210, 2020A1515011408), Guangdong Provincial Science and

5 Technology Program (2019B030301009), the Shenzhen Municipal Government of

6 China (JCYJ20180507182427559, GJHZ20180418190559891), and Shenzhen Key

7 Medical Discipline Construction Fund (SZXK060). Plasmid pCMV-myc-ubiquitin was

8 a gift from Prof. Xingzhi Xu; plasmid pvxl-flag-RabGEF1 was a gift from Dr. Xifeng

9 and plasmid pCDH-puro-KRAS ${ }^{\mathrm{G} 12 \mathrm{D}}$ was a gift from Dr. Hongbin Ji. The authors would

10 like to thank Dr. Xi Chen (SpecAlly Life Technology Co., Ltd, Wuhan, China) for

11 mass spectrometry data analysis, Chengli Weng for animal experimental support, the

12 Instrumental Analysis Center of Shenzhen University (Lihu Campus) for the technical

13 support, and Dr. Jessica Tamanini of ETediting for editing the manuscript prior to

14 submission. 


\section{Author contributions}

2 T.X. and C.C. conceived and designed the experiments. C.C. carried out most of the

3 experiments and analyzed the data. M.Z. helped with the construction of plasmids and

4 lentivirus packaging. T.X. helped with the animal studies. S.F. and H.Z. prepared

5 tissue microarray and performed IHC staining and scoring of SEDT7. C.Z. performed

6 the LC-MS/MS and proteomic analysis. J.Z. performed the computer simulation of

7 SETD7 and KRAS crystal structures. S.L. and W.G. contributed to the planning and

8 discussion of the project. D.Z. supervised the entire project. T.X. and C.C. wrote the

9 manuscript, designed the layout of figures and tables with contributions from all of the

10 authors.

\section{Declaration of interests}

13 All authors declare no competing interests.

14

15 


\section{References}

2 Ahearn, I., Zhou, M., and Philips, M.R. (2018). Posttranslational Modifications of RAS Proteins.

3 Cold Spring Harb Perspect Med 8.

4 Alvarez-Moya, B., Lopez-Alcala, C., Drosten, M., Bachs, O., and Agell, N. (2010). K-Ras4B

5 phosphorylation at Ser181 is inhibited by calmodulin and modulates K-Ras activity and function.

6 Oncogene 29, 5911-5922.

7 Barcelo, C., Etchin, J., Mansour, M.R., Sanda, T., Ginesta, M.M., Sanchez-Arevalo Lobo, V.J., Real,

8 F.X., Capella, G., Estanyol, J.M., Jaumot, M., et al. (2014a). Ribonucleoprotein HNRNPA2B1

9 interacts with and regulates oncogenic KRAS in pancreatic ductal adenocarcinoma cells.

10 Gastroenterology 147, 882-892 e888.

Barcelo, C., Paco, N., Morell, M., Alvarez-Moya, B., Bota-Rabassedas, N., Jaumot, M., Vilardell, F., Capella, G., and Agell, N. (2014b). Phosphorylation at Ser-181 of oncogenic KRAS is required for

13 tumor growth. Cancer Res 74, 1190-1199.

Batista, I.A.A., and Helguero, L.A. (2018). Biological processes and signal transduction pathways regulated by the protein methyltransferase SETD7 and their significance in cancer. Signal Transduct Target Ther 3, 19.

Berman, H.M., Westbrook, J., Feng, Z., Gilliland, G., Bhat, T.N., Weissig, H., Shindyalov, I.N., and Bourne, P.E. (2000). The Protein Data Bank. Nucleic Acids Res 28, 235-242.

Bivona, T.G., Quatela, S.E., Bodemann, B.O., Ahearn, I.M., Soskis, M.J., Mor, A., Miura, J., Wiener, H.H., Wright, L., Saba, S.G., et al. (2006). PKC regulates a farnesyl-electrostatic switch on $\mathrm{K}-\mathrm{Ras}$ that promotes its association with Bcl-XL on mitochondria and induces apoptosis. Mol Cell 21, 481-493.

Brose, M.S., Volpe, P., Feldman, M., Kumar, M., Rishi, I., Gerrero, R., Einhorn, E., Herlyn, M., Minna, J., Nicholson, A., et al. (2002). BRAF and RAS mutations in human lung cancer and melanoma. Cancer Res 62, 6997-7000. vivo. Mol Cell 43, 681-688. 
1 Prives, C., Gamblin, S.J., et al. (2004). Regulation of p53 activity through lysine methylation.

2 Nature 432, 353-360.

3 Copeland, R.A., Solomon, M.E., and Richon, V.M. (2009). Protein methyltransferases as a target

4 class for drug discovery. Nat Rev Drug Discov 8, 724-732.

5 Cox, A.D., Fesik, S.W., Kimmelman, A.C., Luo, J., and Der, C.J. (2014). Drugging the undruggable

6 RAS: Mission possible? Nat Rev Drug Discov 13, 828-851.

7 Del Rizzo, P.A., and Trievel, R.C. (2011). Substrate and product specificities of SET domain

8 methyltransferases. Epigenetics 6, 1059-1067.

9 Ding, L., Getz, G., Wheeler, D.A., Mardis, E.R., McLellan, M.D., Cibulskis, K., Sougnez, C.,

10 Greulich, H., Muzny, D.M., Morgan, M.B., et al. (2008). Somatic mutations affect key pathways in 11 lung adenocarcinoma. Nature 455, 1069-1075.

12 Ea, C.K., and Baltimore, D. (2009). Regulation of NF-kappaB activity through lysine

13 monomethylation of p65. Proc Natl Acad Sci U S A 106, 18972-18977.

14 Fang, Z., Marshall, C.B., Nishikawa, T., Gossert, A.D., Jansen, J.M., Jahnke, W., and Ikura, M.

15 (2018). Inhibition of K-RAS4B by a Unique Mechanism of Action: Stabilizing

16 Membrane-Dependent Occlusion of the Effector-Binding Site. Cell Chem Biol 25, 1327-1336

$17 \mathrm{e} 1324$.

18 Feig, L.A. (2006). The odyssey of k-ras. Mol Cell 21, 447-449.

19 Gyorffy, B., Surowiak, P., Budczies, J., and Lanczky, A. (2013). Online survival analysis software

20 to assess the prognostic value of biomarkers using transcriptomic data in non-small-cell lung cancer.

21 PLoS One 8, e82241.

22 Hancock, J.F., Magee, A.I., Childs, J.E., and Marshall, C.J. (1989). All ras proteins are

23 polyisoprenylated but only some are palmitoylated. Cell 57, 1167-1177.

24 Hancock, J.F., Paterson, H., and Marshall, C.J. (1990). A polybasic domain or palmitoylation is

25 required in addition to the CAAX motif to localize p21ras to the plasma membrane. Cell 63,

$26 \quad 133-139$.

27 Hobbs, G.A., Der, C.J., and Rossman, K.L. (2016). RAS isoforms and mutations in cancer at a

28 glance. J Cell Sci 129, 1287-1292.

29 Hong, X., Huang, H., Qiu, X., Ding, Z., Feng, X., Zhu, Y., Zhuo, H., Hou, J., Zhao, J., Cai, W., et al. 30 (2018). Targeting posttranslational modifications of RIOK1 inhibits the progression of colorectal 31 and gastric cancers. Elife 7.

32 Kim, Y., Nam, H.J., Lee, J., Park, D.Y., Kim, C., Yu, Y.S., Kim, D., Park, S.W., Bhin, J., Hwang, D., 
1 et al. (2016). Methylation-dependent regulation of HIF-1alpha stability restricts retinal and tumour 2 angiogenesis. Nat Commun 7, 10347.

3 Kurash, J.K., Lei, H., Shen, Q., Marston, W.L., Granda, B.W., Fan, H., Wall, D., Li, E., and Gaudet,

4 F. (2008). Methylation of p53 by Set7/9 mediates p53 acetylation and activity in vivo. Mol Cell 29,

$5 \quad 392-400$.

6 Liu, X., Chen, Z., Xu, C., Leng, X., Cao, H., Ouyang, G., and Xiao, W. (2015). Repression of

7 hypoxia-inducible factor alpha signaling by Set7-mediated methylation. Nucleic Acids Res 43,

$8 \quad 5081-5098$.

9 Montenegro, M.F., Sanchez-Del-Campo, L., Gonzalez-Guerrero, R., Martinez-Barba, E.,

10 Pinero-Madrona, A., Cabezas-Herrera, J., and Rodriguez-Lopez, J.N. (2016). Tumor suppressor

11 SET9 guides the epigenetic plasticity of breast cancer cells and serves as an early-stage biomarker

12 for predicting metastasis. Oncogene 35, 6143-6152.

13 Nishioka, K., Chuikov, S., Sarma, K., Erdjument-Bromage, H., Allis, C.D., Tempst, P., and

14 Reinberg, D. (2002). Set9, a novel histone H3 methyltransferase that facilitates transcription by

15 precluding histone tail modifications required for heterochromatin formation. Genes Dev 16,

16 479-489.

17 Oudhoff, M.J., Braam, M.J.S., Freeman, S.A., Wong, D., Rattray, D.G., Wang, J., Antignano, F.,

18 Snyder, K., Refaeli, I., Hughes, M.R., et al. (2016). SETD7 Controls Intestinal Regeneration and

19 Tumorigenesis by Regulating Wnt/beta-Catenin and Hippo/YAP Signaling. Dev Cell 37, 47-57.

20 Plowman, S.J., Ariotti, N., Goodall, A., Parton, R.G., and Hancock, J.F. (2008). Electrostatic

21 interactions positively regulate K-Ras nanocluster formation and function. Mol Cell Biol 28,

$22 \quad 4377-4385$.

23 Prior, I.A., Lewis, P.D., and Mattos, C. (2012). A comprehensive survey of Ras mutations in cancer.

24 Cancer Res 72, 2457-2467.

25 Scheffzek, K., Ahmadian, M.R., Kabsch, W., Wiesmuller, L., Lautwein, A., Schmitz, F., and

26 Wittinghofer, A. (1997). The Ras-RasGAP complex: structural basis for GTPase activation and its

27 loss in oncogenic Ras mutants. Science 277, 333-338.

28 Shen, C., Wang, D., Liu, X., Gu, B., Du, Y., Wei, F.Z., Cao, L.L., Song, B., Lu, X., Yang, Q., et al.

29 (2015). SET7/9 regulates cancer cell proliferation by influencing beta-catenin stability. FASEB J 29, $30 \quad 4313-4323$.

31 Simanshu, D.K., Nissley, D.V., and McCormick, F. (2017). RAS Proteins and Their Regulators in 32 Human Disease. Cell 170, 17-33.

33 Subramanian, K., Jia, D., Kapoor-Vazirani, P., Powell, D.R., Collins, R.E., Sharma, D., Peng, J., 
1 Cheng, X., and Vertino, P.M. (2008). Regulation of estrogen receptor alpha by the SET7 lysine

2 methyltransferase. Mol Cell 30, 336-347.

3 Tian, T., Harding, A., Inder, K., Plowman, S., Parton, R.G., and Hancock, J.F. (2007). Plasma

4 membrane nanoswitches generate high-fidelity Ras signal transduction. Nat Cell Biol 9, 905-914.

5 Trahey, M., and McCormick, F. (1987). A cytoplasmic protein stimulates normal N-ras p21 GTPase,

6 but does not affect oncogenic mutants. Science 238, 542-545.

7 Tricker, E.M., Xu, C., Uddin, S., Capelletti, M., Ercan, D., Ogino, A., Pratilas, C.A., Rosen, N.,

8 Gray, N.S., Wong, K.K., et al. (2015). Combined EGFR/MEK Inhibition Prevents the Emergence

9 of Resistance in EGFR-Mutant Lung Cancer. Cancer Discov 5, 960-971.

10 Wang, C., Shu, L., Zhang, C., Li, W., Wu, R., Guo, Y., Yang, Y., and Kong, A.N. (2018). Histone

11 Methyltransferase Setd7 Regulates Nrf2 Signaling Pathway by Phenethyl Isothiocyanate and

12 Ursolic Acid in Human Prostate Cancer Cells. Mol Nutr Food Res 62, e1700840.

13 Wang, H., Cao, R., Xia, L., Erdjument-Bromage, H., Borchers, C., Tempst, P., and Zhang, Y. (2001).

14 Purification and functional characterization of a histone H3-lysine 4-specific methyltransferase.

15 Mol Cell 8, 1207-1217.

16 Wang, M.T., Holderfield, M., Galeas, J., Delrosario, R., To, M.D., Balmain, A., and McCormick, F. 17 (2015). K-Ras Promotes Tumorigenicity through Suppression of Non-canonical Wnt Signaling.

18 Cell 163, 1237-1251.

19 Xiao, B., Jing, C., Wilson, J.R., Walker, P.A., Vasisht, N., Kelly, G., Howell, S., Taylor, I.A.,

20 Blackburn, G.M., and Gamblin, S.J. (2003). Structure and catalytic mechanism of the human

21 histone methyltransferase SET7/9. Nature 421, 652-656.

22 Xu, L., Lubkov, V., Taylor, L.J., and Bar-Sagi, D. (2010). Feedback regulation of Ras signaling by

23 Rabex-5-mediated ubiquitination. Curr Biol 20, 1372-1377.

24 Yang, X.D., Huang, B., Li, M., Lamb, A., Kelleher, N.L., and Chen, L.F. (2009). Negative

25 regulation of NF-kappaB action by Set9-mediated lysine methylation of the RelA subunit. EMBO J

$26 \quad 28,1055-1066$.

27 Zhou, Y., Prakash, P., Liang, H., Cho, K.J., Gorfe, A.A., and Hancock, J.F. (2017). Lipid-Sorting

28 Specificity Encoded in K-Ras Membrane Anchor Regulates Signal Output. Cell 168, 239-251 e216.

29

30

31

32

33 


\section{Figure Legends}

\section{Figure 1. SETD7 interacts with KRAS}

3 (A) Proteins from the co-immunoprecipitation (co-IP) assay using anti-HA magnetic

4 beads in $293 \mathrm{~T}$ with ectopic expression of $3 \times$ HA-tagged KRAS were separated by

5 polyacrylamide gel electrophoresis and visualized using silver staining. (B) The

6 workflow for identifying KRAS-interacting proteins was shown, including co-IP assay,

7 sample preparation, LC-MS/MS analysis, protein quantification, and further

8 computational analyses for differential proteins. (C) Volcano plot of proteins identified

9 by LC-MS/MS analysis in HA-vector and 3×HA-KRAS groups. The ratio (KRAS/HA)

10 and the mean of protein spectra counts (MeanSP) in two groups were calculated. The $\mathrm{x}$

11 axis shows the $\log 2$ ratio (KRAS/HA) and the y axis shows the $\log 2$ MeanSP. The

12 proteins distributed outside the dark-red boundary line $\left(\mathrm{y}=\log _{2} 2.5 /(\mathrm{x}-1)\right)$ were

13 labeled as significant differential proteins in KRAS group. TOP 10 differential proteins

14 from each group were labeled. (D) Western blotting (WB) analysis of whole-cell

15 lysates and immunoprecipitation (IP) of SETD7 in 293T cells transfected with the

16 indicated plasmids. (E) Immunofluorescence staining of SETD7 (green) and KRAS

17 (red) in 293T cells co-transfected with SETD7 and KRAS. DAPI was used to label the

18 nuclei. Scale bar, $10 \mu \mathrm{m}$. (F and G) WB analysis of the co-IP assay using anti-SETD7

19 (F) or anti-RAS (G) antibodies in H358-SETD7-O/E stable cells. (H) WB analysis of 
1 the co-IP assay with anti-SETD7 antibody in non-small-cell lung cancer (NSCLC) cell

2 line A549.

4 Figure 2. SETD7 plays a tumor-suppressive role in non-small-cell lung cancer

$5 \quad$ (NSCLC) cell lines by downregulating RAS-associated signaling.

6 (A-C) Cell proliferation (A), two-dimensional (2-D) colony formation (B), and

7 anchorage-independent cell growth (C) of H358-vector and H358-SETD7-O/E stable

8 cells were measured separately. (D) Western blotting (WB) analysis of SETD7 and

9 RAS-related signaling pathways in H358 stable cells. (E-G) Cell proliferation (E), 2-D

10 colony formation (F), and anchorage-independent cell growth (G) of H1437-control

11 and two SETD7-KD stable cells were measured separately. The bar graphs represent

12 the quantification of numbers of colonies on plates or spheres in soft agar from three

13 independent experiments. (H) WB analysis of SETD7 and RAS-related signaling

14 pathways in $\mathrm{H} 1437$ stable cells. $* * P<0.01 ; * * * P<0.001$.

\section{Figure 3. The anti-cancer effect of SETD7 in vivo.}

17 (A) Measurement of volumes of xenograft tumors derived from A549-vector or A549-

18 SETD7-O/E stable cells $(\mathrm{n}=8)$. (B) A photograph of the xenograft tumors and the

19 tumor weights, 7 weeks after cell injection, are shown (upper and lower panels, 
1 respectively). (C) Representative images of immunohistochemical (IHC) staining of

2 SETD7, RAS, and Ki67 in xenograft tumor tissues. Scale bar, $100 \mu \mathrm{m}$. (D)

3 Measurement of volumes of xenograft tumors derived from H1437-control and two

4 SETD7-KD stable cells $(\mathrm{n}=10)$. (E) A photograph of the xenograft tumors and the

5 tumor weights, 4 weeks after cell injection, are shown (upper and lower panels,

6 respectively; scale bar, $10 \mathrm{~mm}$ ). (F) Representative images of IHC staining of SETD7,

7 RAS, and Ki67 in xenograft tumor tissues of each group. Scale bar, $100 \mu \mathrm{m} .{ }^{*} P<0.05$;

$8 \quad * * P<0.01 ; * * * P<0.001$.

9

Figure 4. SETD7 promotes KRAS degradation and ubiquitination in a

\section{catalytic-dependent manner.}

(A) Western blotting (WB) analysis of 293T cells transfected with KRAS and SETD7 or KRAS alone with cycloheximide $(\mathrm{CHX})(100 \mu \mathrm{g} / \mathrm{mL})$ treatment, as indicated. (B) WB analysis of H358-vector and H358-SETD7-O/E stable cells with CHX (100 $\mu \mathrm{g} / \mathrm{mL}$ ) treatment, as indicated. (C) WB analysis of H1437-control and two SETD7-KD stable cells with CHX $(100 \mu \mathrm{g} / \mathrm{mL})$ treatment, as indicated. The graphs in A-C represent the quantification of RAS protein levels relative to the $\alpha$-tubulin level. (D) WB analysis of the co-immunoprecipitation (co-IP) assay using anti-RAS antibody in H358 cells stably expressing the SETD7 H297A mutant. (E) WB analysis of 293T 
1 cells co-transfected with KRAS and wild-type SETD7 or SETD7 H297A mutant with

2 CHX $(100 \mu \mathrm{g} / \mathrm{mL})$ treatment, as indicated. The graph represents the quantification of

3 RAS protein levels relative to the $\alpha$-tubulin level. (F) 293 T cells transfected with the

4 indicated combinations of KRAS, HA-SETD7 (WT or H297A), and myc-Ubi were

5 subjected to a ubiquitination assay. (G-I) Cell proliferation (G), 2-D colony formation

6 (H) and spheroid growth (I) of H358-vector, H358-SETD7 ${ }^{\mathrm{WT}}$, and H358-SETD7 ${ }^{\mathrm{H} 297 \mathrm{~A}}$

7 stable cells were measured separately. $* P<0.05 ; * * P<0.01 ; * * * P<0.001$. (J) WB

8 analysis of SETD7 and RAS-related signaling pathways in H358 stable cells, as 9 indicated.

11 Figure 5. SETD7 methylates KRAS at K182 and K184, leading to KRAS 12 ubiquitination and degradation via recruitment of RabGEF1

13 (A) Alignment of amino acid sequences from proteins reported to be SETD7 substrates

14 and amino acids 178-186 in the C-terminal of KRAS. Lysines 182 and 184 of KRAS

15 were predicted to be potential residues for SETD7-mediated methylation. (B) A

16 representative structure of the interactional network between SETD7 and KRAS, based

17 on a cluster analysis. K182, T183, and K184 of KRAS and some important residues of

18 SETD7 are represented by a stick model. Residues from SETD7 and KRAS are

19 colored orange and cyan, respectively. (C) $293 \mathrm{~T}$ cells were co-transfected with 
1 KRAS-myc and wild-type SETD7 or SETD7 H297A mutant. KRAS was

2 immunoprecipitated (IP) using anti-myc antibody and the methylation level of KRAS

3 was detected using mono-methyl lysine (mme-K) antibody. (D) KRAS methylation

4 was detected in 293 T cells transfected with the indicated plasmids and IP using

5 anti-RAS antibody. (E) KRAS peptide modified with methylation on lysine 182

6 residue was identified by LC-MS/MS. (F) Western blotting (WB) analysis of

7 whole-cell lysates (WCLs) and IP of RAS in 293T cells transfected with the indicated

8 plasmids. (G) WB analysis of 293 T cells transfected with combinations of RabGEF1,

9 HA-SETD7, and KRAS (WT, K182/184M, or G12D) with CHX (100 $\mu \mathrm{g} / \mathrm{mL})$

10 treatment, as indicated. The graph represents the quantification of RAS protein levels

11 relative to the $\alpha$-tubulin level. (H) $293 \mathrm{~T}$ cells transfected with the indicated

12 combinations of RabGEF1, HA-SETD7, KRAS (WT, K182/184M, or G12D), and

13 myc-Ubi were subjected to a ubiquitination assay. (I) Cell proliferation of

14 H1437-vector, H1437-KRAS ${ }^{\mathrm{G} 12 \mathrm{D}}$, and H1437-KRAS ${ }^{\mathrm{G} 12 \mathrm{D} / \mathrm{K} 182 / 184 \mathrm{M}}$ stable cells was

15 measured using a CCK-8 assay. $* P<0.05 ; * * P<0.01$. (J) WB analysis of SETD7 and

16 RAS-related signaling pathways in H1437 stable cells, as indicated.

18 Figure 6. The clinical significance of SETD7 in lung adenocarcinoma (LUAD)

19 (A) Kaplan-Meier plotter survival analysis for SETD7 mRNA levels (probe set, 
1 224928_at) in lung cancer $(\mathrm{n}=1144)$ and LUAD $(\mathrm{n}=672)$ patient samples. Medium

2 was used as a cutoff to divide the samples into low and high groups. (B) Representative

3 images of immunohistochemical (IHC) staining of SETD7 in a cohort of 127 LUAD

4 tissue samples. The staining intensity was classified as negative (-), weak

$5(+)$, moderate $(++)$, or strong $(+++)$. (C) Survival analysis for IHC staining intensity of

6 SETD7 in LUAD tissue samples. Negative and weak staining was indicated as

7 SETD7-low $(\mathrm{n}=52)$, while moderate and strong staining was indicated as SETD7-high

$8 \quad(\mathrm{n}=75)$.

9

10 Figure 7. Proposed mechanism of SETD7-mediated KRAS methylation and

11

12

13 ligase, RABGEF1, is recruited in a methylation-dependent manner and promotes

14 polyubiquitination and degradation of KRAS. A reduction in KRAS protein levels

15

16

17

18

\section{degradation in lung cancer}

SETD7 interacts with KRAS and methylates KRAS at K182 and K184. Then, the E3

suppresses the downstream MEK/ERK and PI3K/AKT cascades and inhibits tumor

progression. In lung adenocarcinoma, SETD7 is frequently downregulated, which

abolishes its inhibitory effect on KRAS and emancipates the oncogenic role of KRAS. 


\section{Supplementary Figure Legends}

2 Figure S1. Correlation between SETD7 expression and tumor progression in

3 non-small-cell lung cancer (NSCLC) cell lines and clinical specimens

4 (A) Two representative MS2 spectra of SETD7 peptides (116-143 and 324-344) from

$53 \times$ HA-KRAS group. (B) Western blotting (WB) analysis of SETD7 expression in

6 NSCLC cell lines from the American Type Culture Collection (ATCC). Staging

7 information is shown above each cell line in red font.

8

9

10

11

Figure S2. The tumor-suppressive role of SETD7 in NSCLC cell lines

(A-B) Cell proliferation (A) and two-dimensional (2-D) colony formation (B) of A549-vector and A549-SETD7-OE stable cells was measured separately. (C) WB analysis of SETD7 and RAS-related signaling pathways in A549 stable cells. (D-E)

Cell proliferation (D), 2-D colony formation (E) of H358-control and two SETD7-KD stable cells were measured separately. (F-G) 3-D spheroid growth of H1437 (F) and H358 (G) controls and two SETD7-KD stable cells was measured separately. (H) WB analysis of SETD7 and RAS-related signaling pathways in H358 stable cells. ${ }^{*} P<0.05$;

$* * P<0.01 ; * * * P<0.001$.

\section{Figure S3. The anti-cancer effect of SETD7 in a xenograft assay}


1 (A) Tumor volumes of xenografts derived from A549 vector and SETD7-OE stable

2 pools were measured 7 weeks after tumor cell injection. $* * P<0.01$. (B) Tumor volumes

3 of xenografts derived from H1437 vector and two SETD7-KD stable pools were

4 measured 4 weeks after tumor cell injection. $* * * P<0.001$.

6 Figure S4. The anti-cancer effect of SETD7 is dependent on its catalytic activity

7 (A-C) KRAS mRNA expression was detected by qPCR in A549 (A) and H358 (B)

8 stable cells with SETD7 overexpression, or H1437 (C) stable cells with SETD7

9 knockdown. (D) WB analysis of A549-vector and A549-SETD7-O/E stable cells with

$10 \mathrm{CHX}(100 \mu \mathrm{g} / \mathrm{mL})$ treatment, as indicated. The relative RAS protein level is labeled in

11 red. (E) Cell proliferation of A549 vector, SETD7 ${ }^{\mathrm{WT}}$, and SETD7 ${ }^{\mathrm{H} 297 \mathrm{~A}}$ stable cells was

12 measured using a CCK-8 assay. (F-G) Two-dimensional (2-D) colony formation (F)

13 and 3-D spheroid growth (G) of A549 vector, SETD7 ${ }^{\text {WT }}$, and SETD7 ${ }^{\text {H297A }}$ stable cells

14 were measured separately. The bar graphs represent the quantification of numbers of

15 colonies on plates or spheroids in dishes with a cell-repellent surface, from three

16 independent experiments. (H) WB analysis of SETD7 and RAS-related signaling

17 pathways in A549 stable cells. $* P<0.05$, $* * * P<0.001 . \mathrm{ns}$, not significant. 


\section{Figure S5. The interaction between SETD7 and KRAS}

2 (A) A computer simulation of the interaction between SETD7 and KRAS. The SETD7

3 sequence G117-K366 was applied to perform the molecular dynamics (MD) simulation.

4 Binding sites of the SETD7 SET domain (PDB ID: 1O9S): TYR245, GLY264,

5 ASN265, THR266, LEU267, SER268, HIS293, ALA295, TYR305, TYR335, GLY336,

6 TYR337 $(245,264,265,266,267,268,293,295,305,335,336,337)$; Prediction of

7 the methylation site of KRAS (PDB ID: 5OCG): K182 or K184 (181, 182, 183, 184,

8 185). The listed structures from HADDOCK were selected as the initial structures for

9 MD simulations. (B) The root-mean-square deviation (RMSD) calculated for the

10 backbone atoms evolved with the simulation time. (C) KRAS peptide modified with

11 methylation on lysine 182 residue was identified by LC-MS/MS. (D) WB analysis of

12 293T cells transfected with KRAS and RABGEF1 or KRAS alone with CHX (100

$13 \mu \mathrm{g} / \mathrm{mL}$ ) treatment, as indicated. (E) WB analysis of $293 \mathrm{~T}$ cells co-transfected with

14 KRAS and SETD7 with or without RabGEF1, following indicated CHX $(100 \mu \mathrm{g} / \mathrm{mL})$

15 treatment. (F) WB analysis of 293T cells co-transfected with KRAS and SETD7, and

16 control or RabGEF1 shRNAs, following indicated CHX $(100 \mu \mathrm{g} / \mathrm{mL})$ treatment (upper

17 panel). Knockdown efficiency of RabGEF1 was monitored by qPCR (lower panel). 


\section{1 mRNA levels in lung cancer and lung adenocarcinoma (LUAD)}

2 (A) KM plotter survival analysis for SETD7 mRNA in patients with lung squamous

3 cell carcinoma $(n=271)$. (B) KM plotter survival analysis for RABGEF1 mRNA

4 levels (probe set, 218310_at) in lung cancer $(\mathrm{n}=1925)$ and LUAD $(\mathrm{n}=720)$ patient

5 samples. (C-G) KM plotter survival analysis for the combination of SETD7 and

6 RABGEF1 mRNA levels in patients with LUAD. The comparisons were performed

7 between patients with both high SETD7 and RabGEF1 expression $(n=336)$ and

8 patients with both low SETD7 and RabGEF1 expression $(n=336)(C)$; Patients with

9 high SETD7 expression were divided into two groups according to whether their

10 RABGEF1 expression was high $(\mathrm{n}=167)$ or low $(\mathrm{n}=168)(\mathrm{D})$; Patients with high

11 RABGEF1 expression were divided into two groups according to SETD7 expression,

12 high $(\mathrm{n}=167)$ or low $(\mathrm{n}=166)(\mathrm{E})$; Patients with low SETD7 expression were divided

13 into two groups according to RABGEF1 expression, high $(\mathrm{n}=166)$ or low $(\mathrm{n}=169)$

14 (F); Patients with low RABGEF1 expression were divided into two groups according

15 to SETD7 expression, high $(\mathrm{n}=167)$ or low $(\mathrm{n}=168)(\mathrm{G})$. In the above KM plotter

16 survival analyses, medium was used as a cutoff to divide the samples into low and high

17 groups. 
A

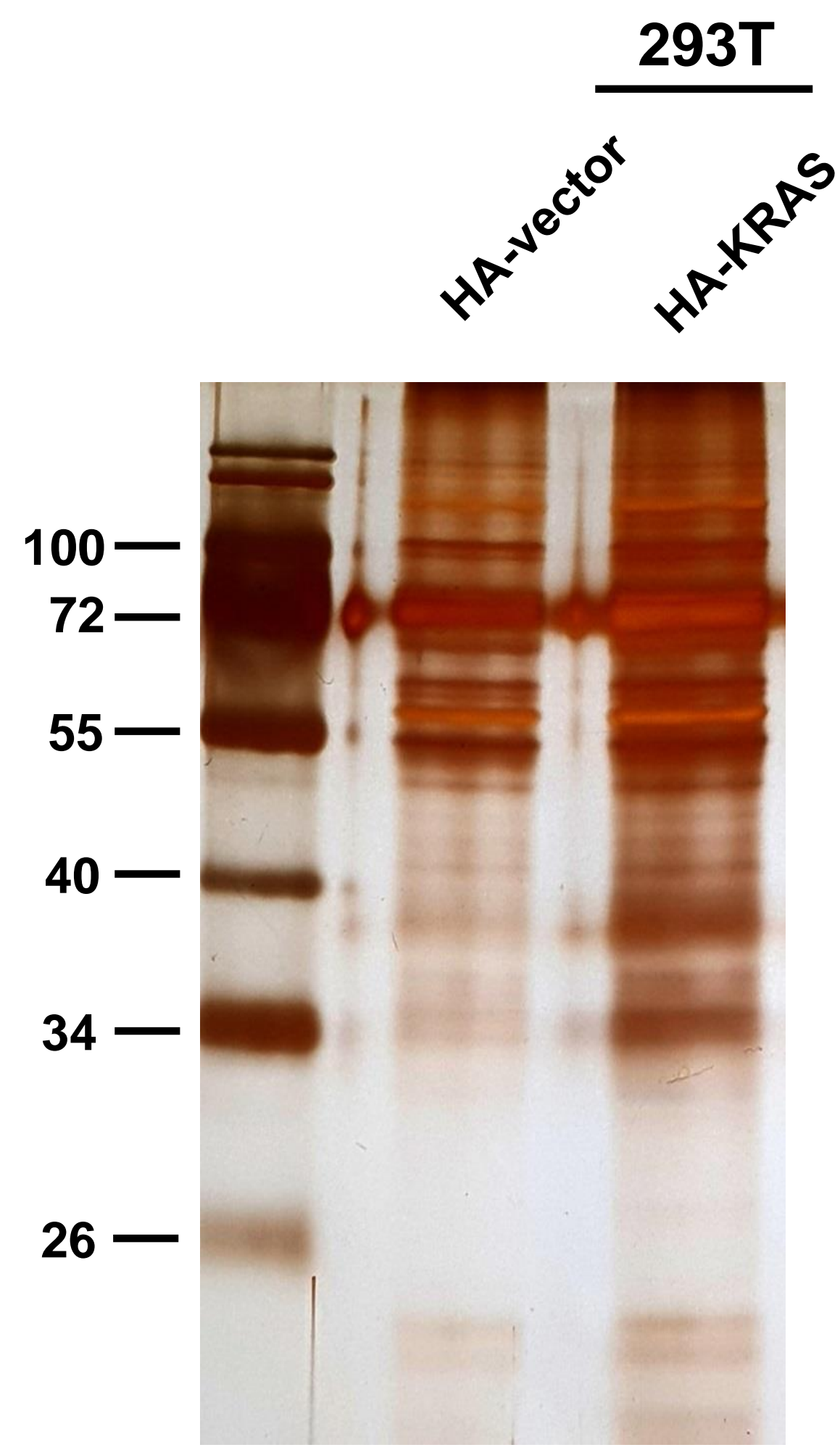

C
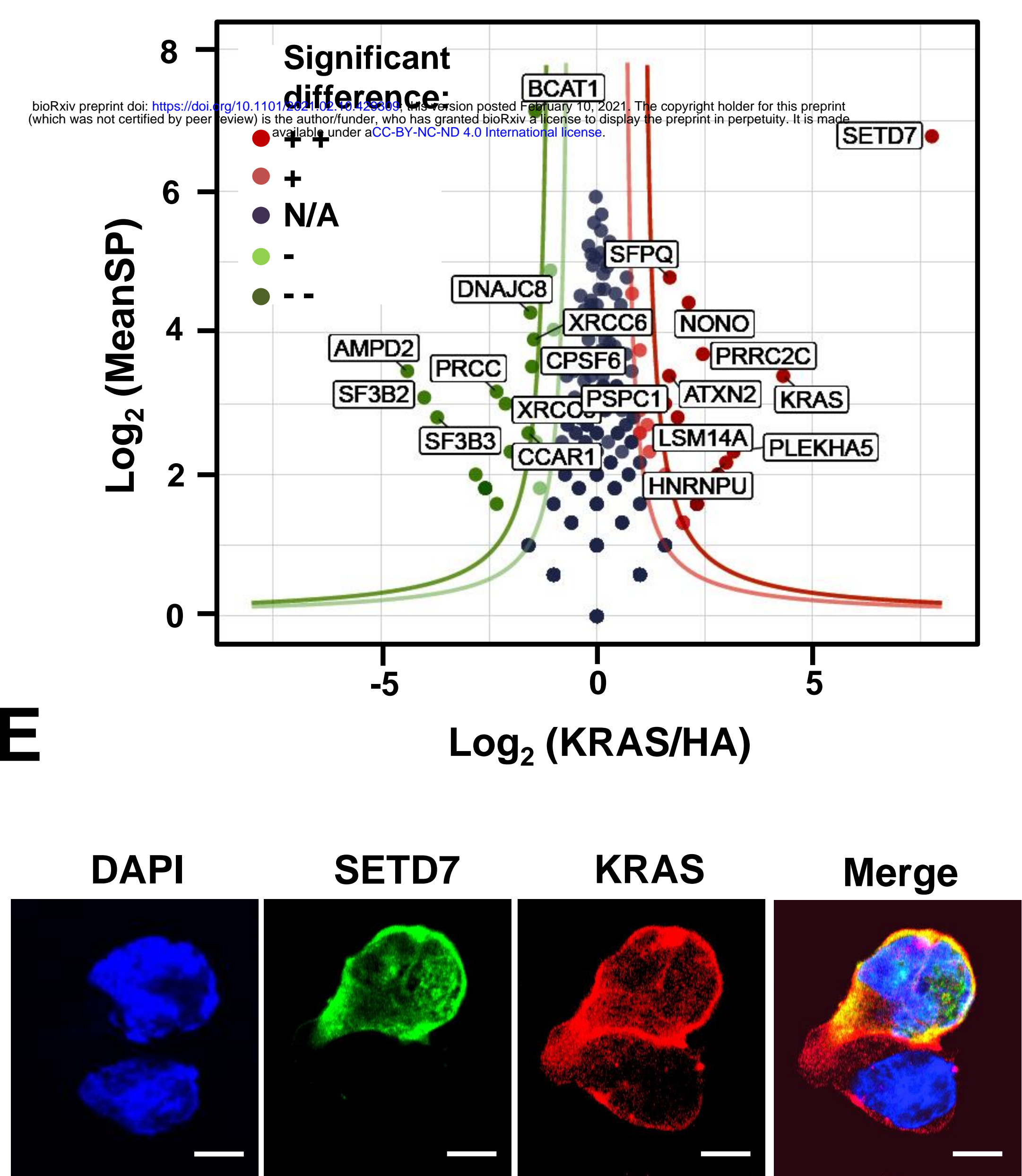

293T-Vector

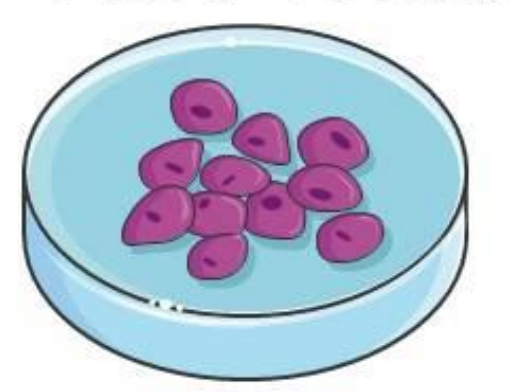

293T-3xHAKRAS

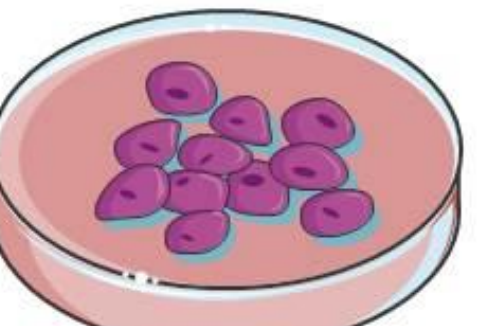

D

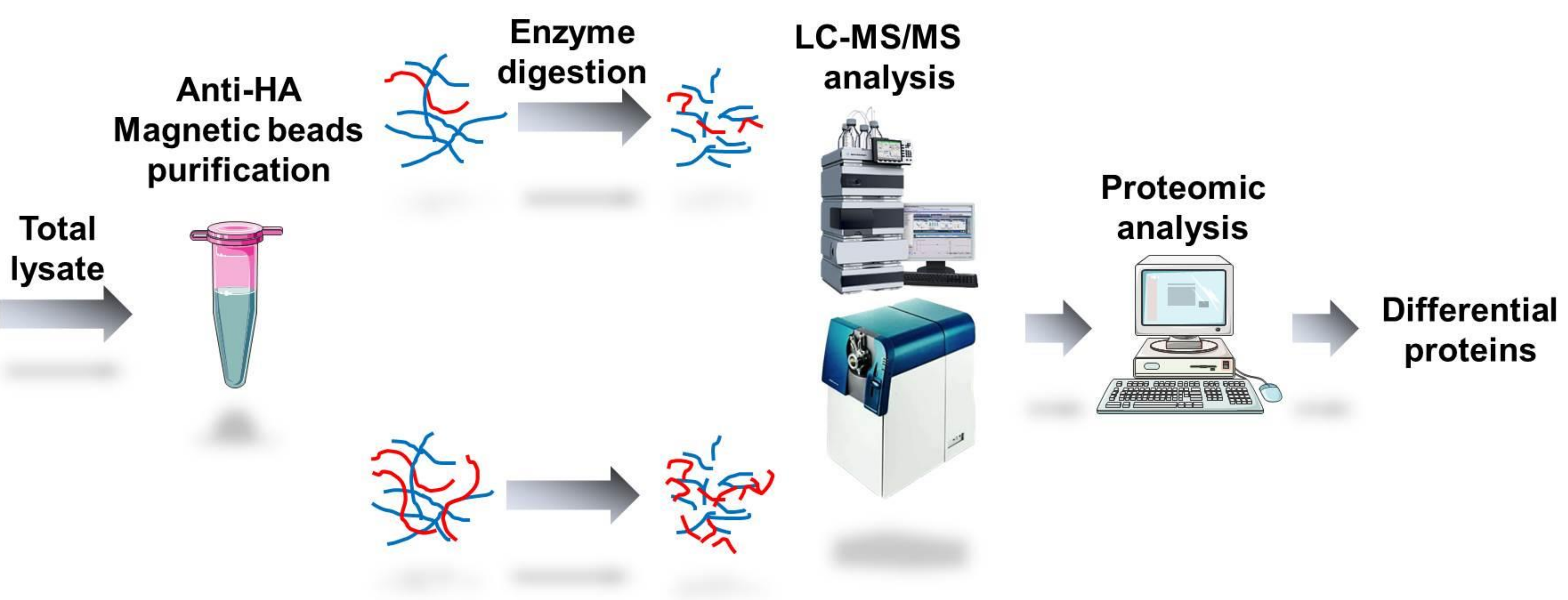

F

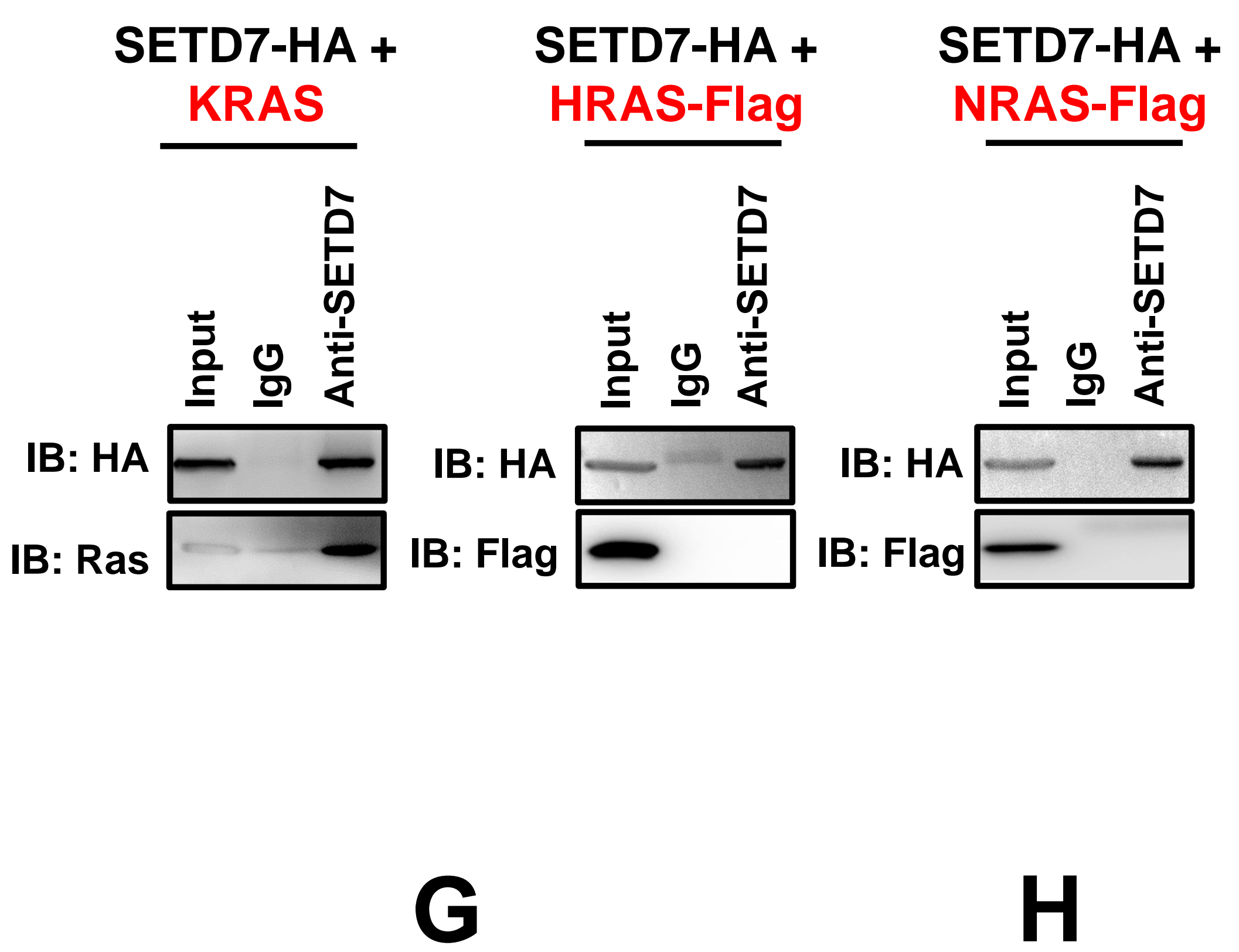

H358

SETD7WT_OE
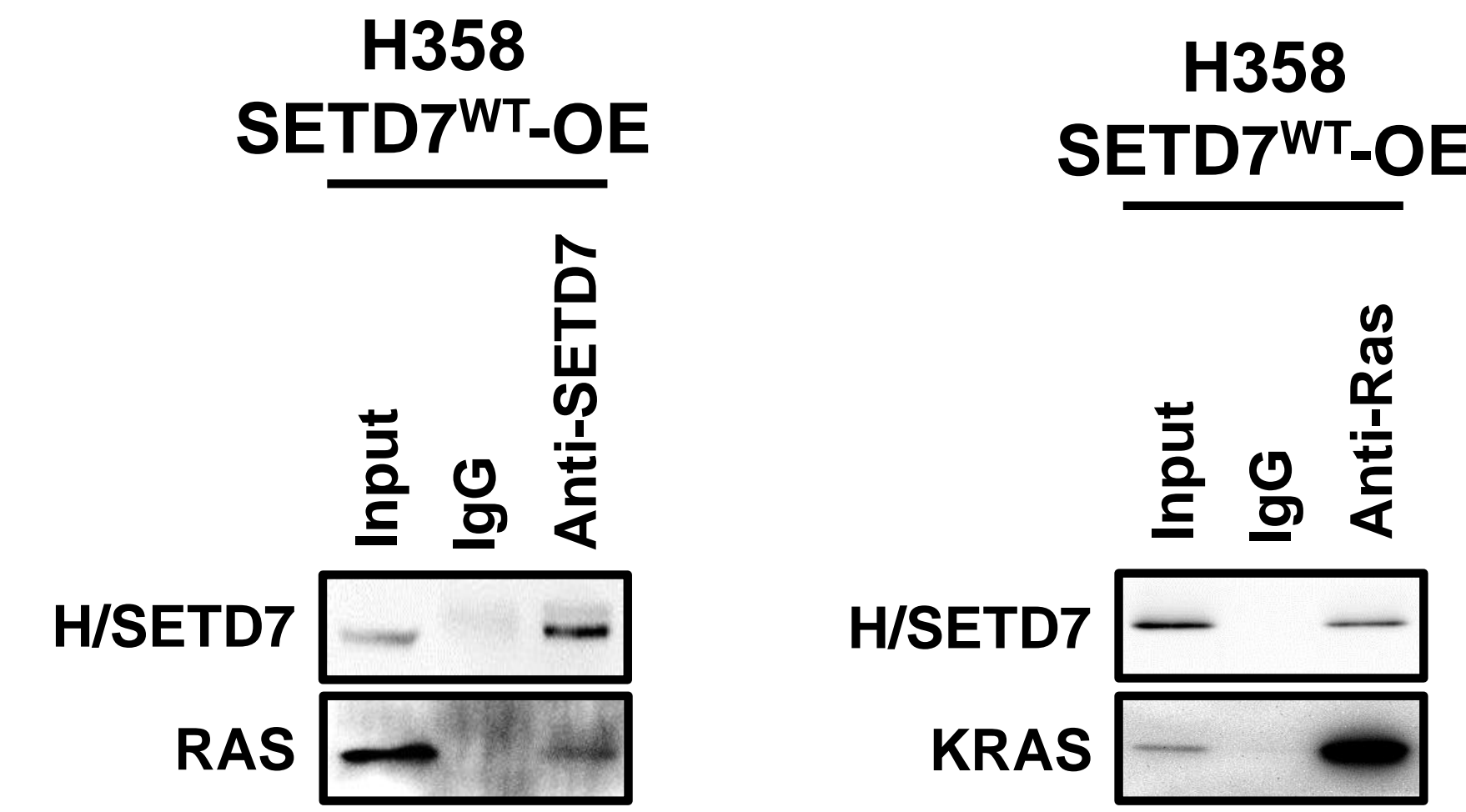


\section{Figure S1}

\section{A}
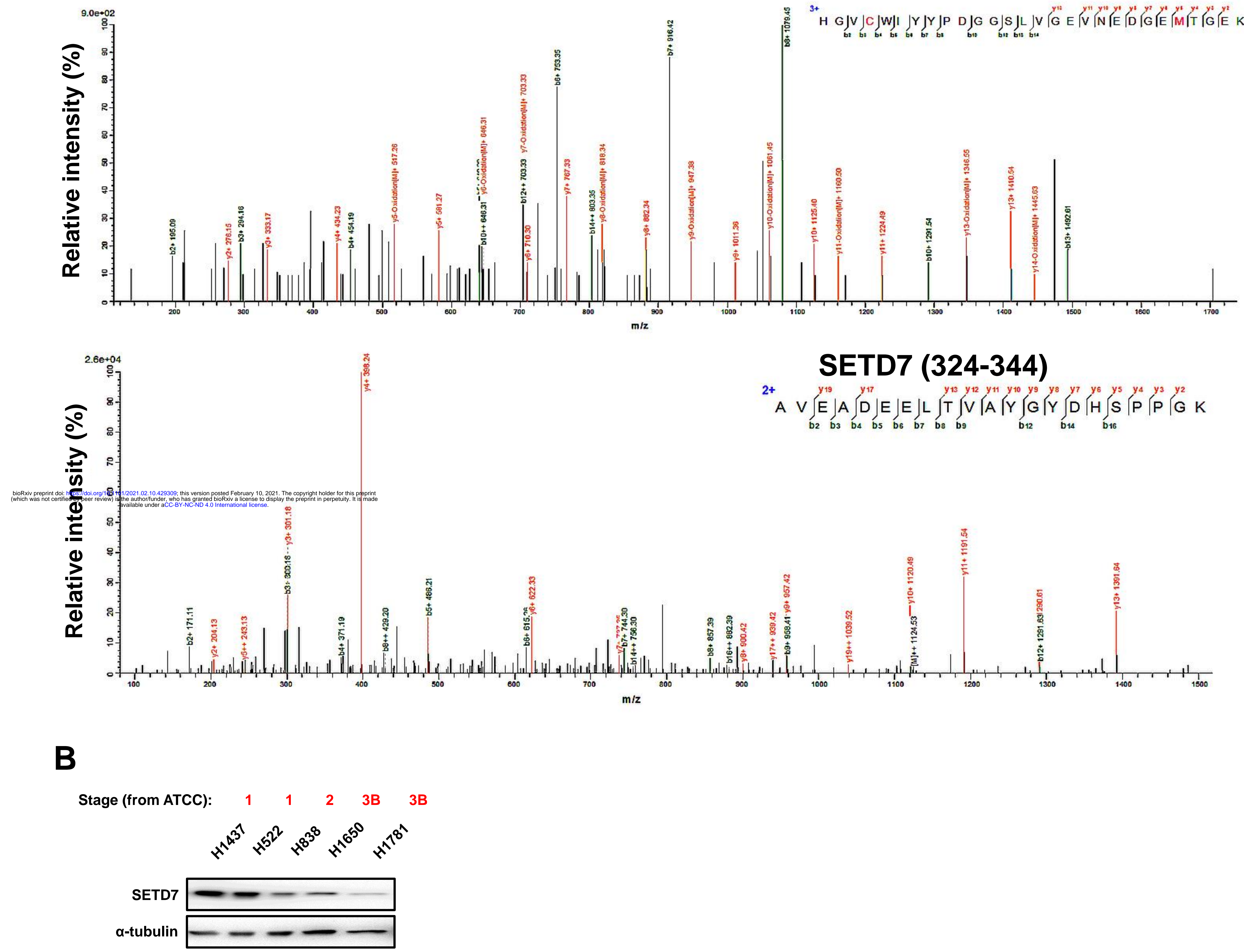

\section{SETD7 (116-143)}

\section{SETD7 (324-344)}

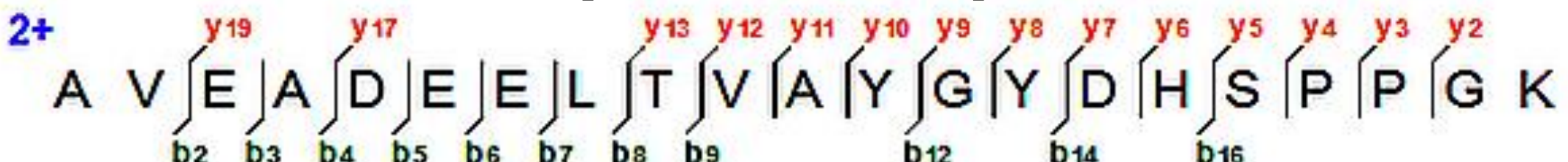

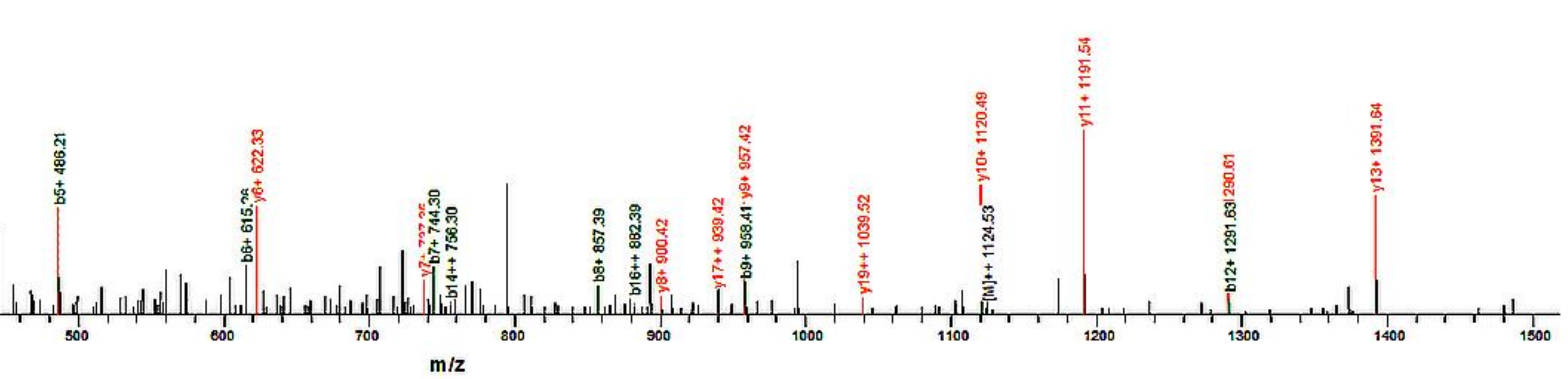




\section{Figure 2}

A

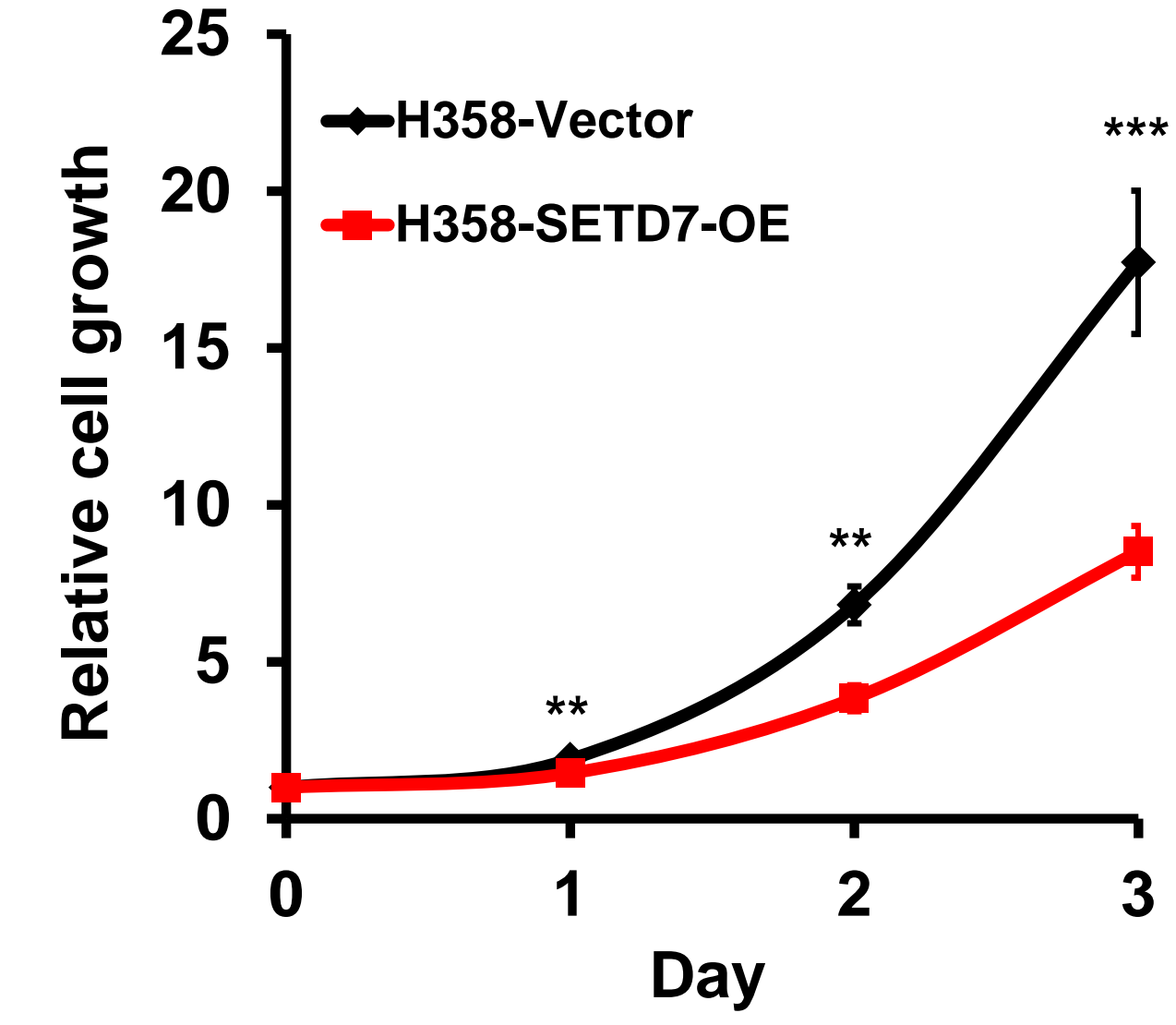

E

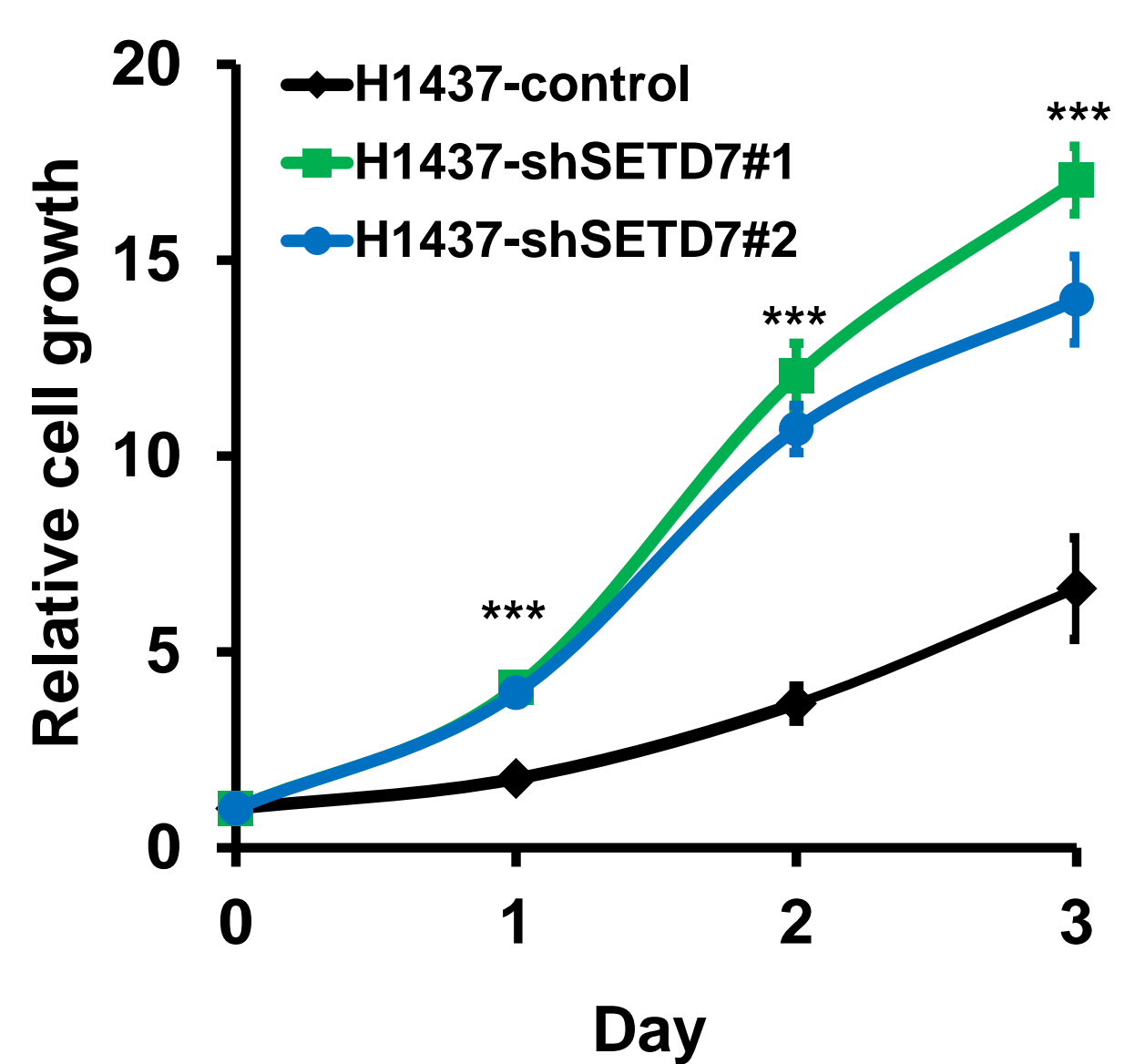

B

C
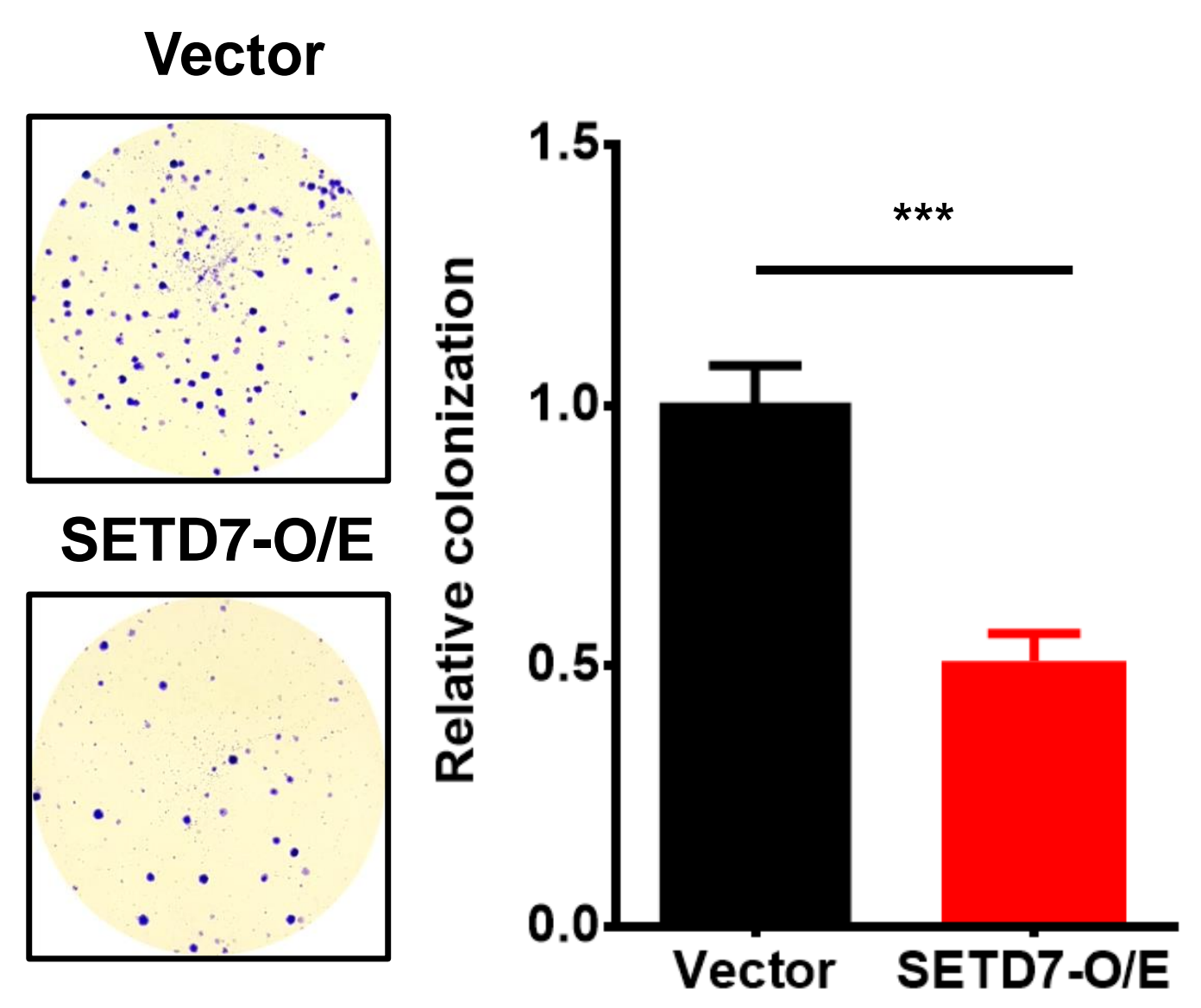

F

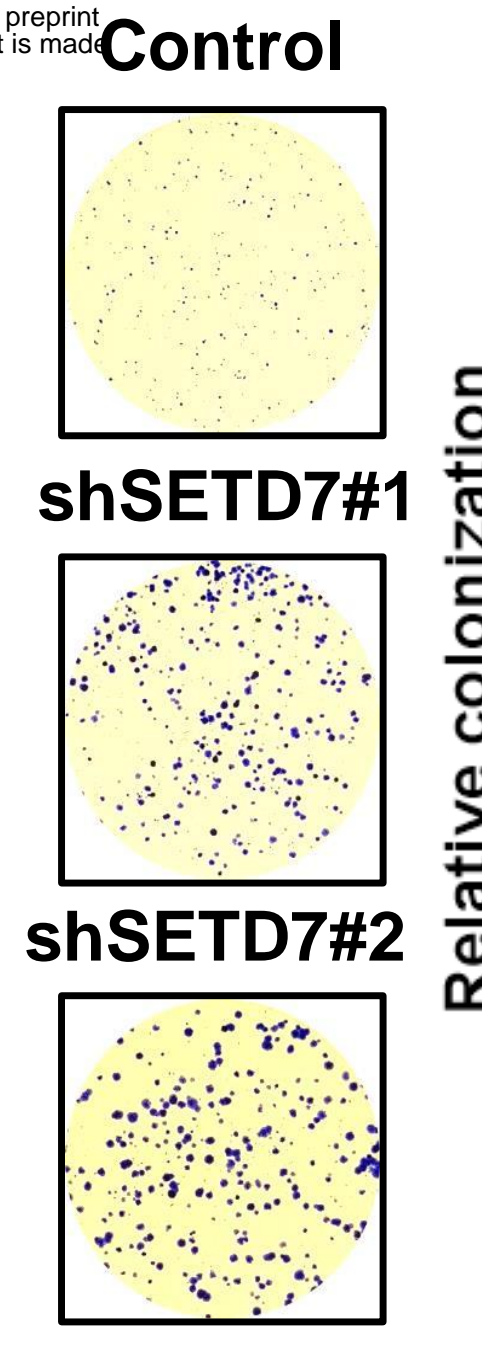

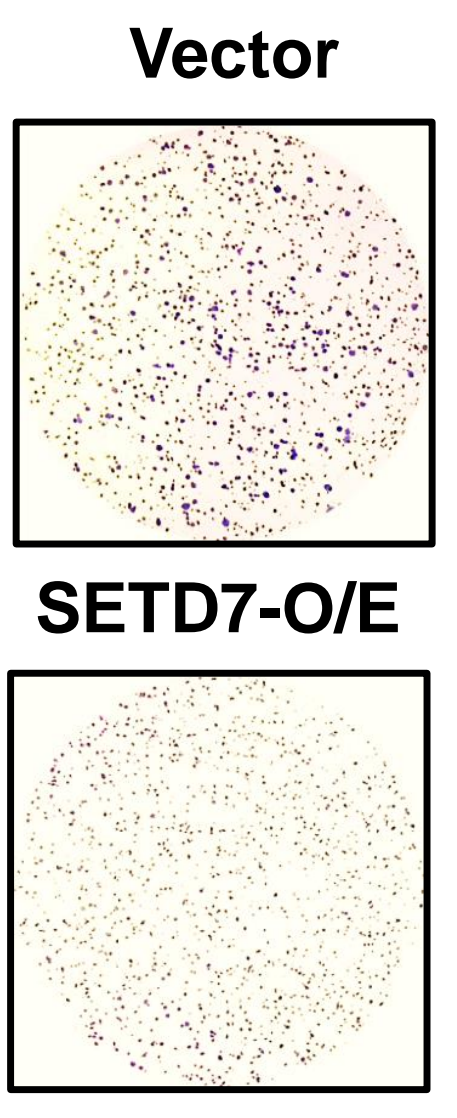

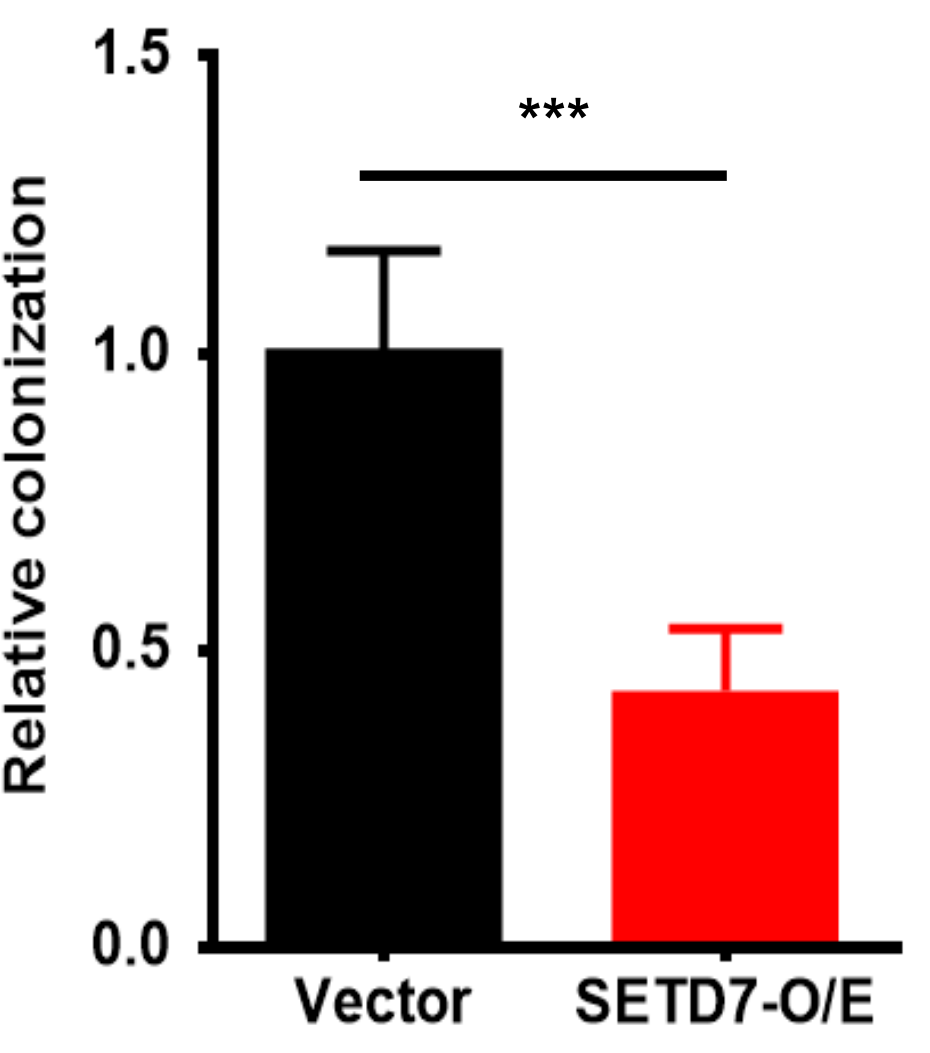

G

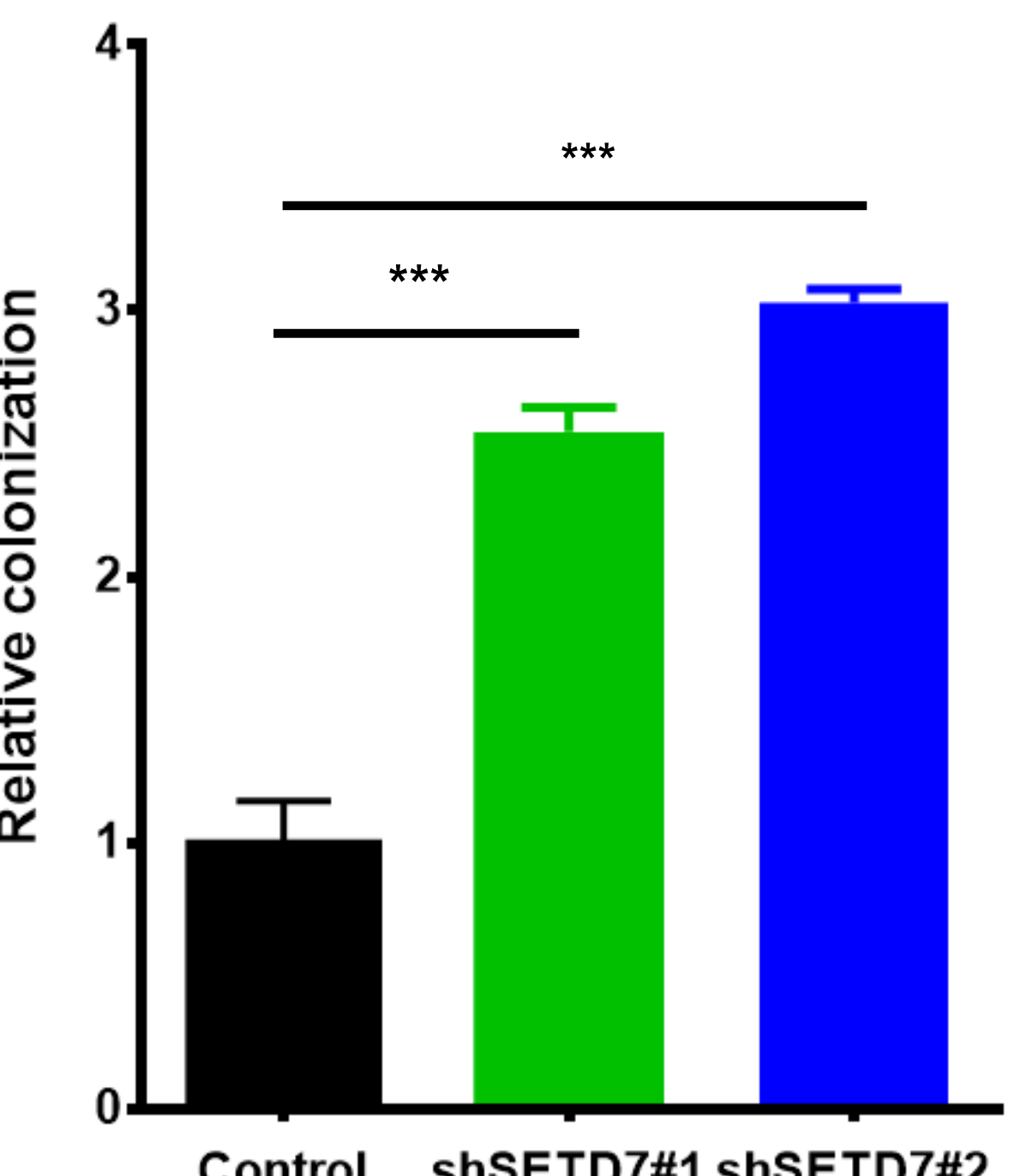

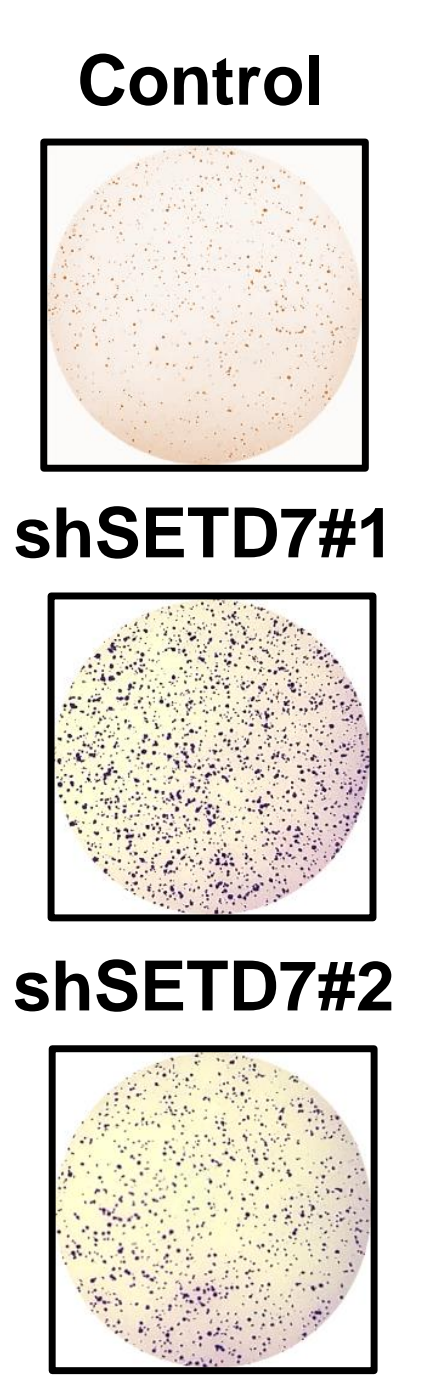

D

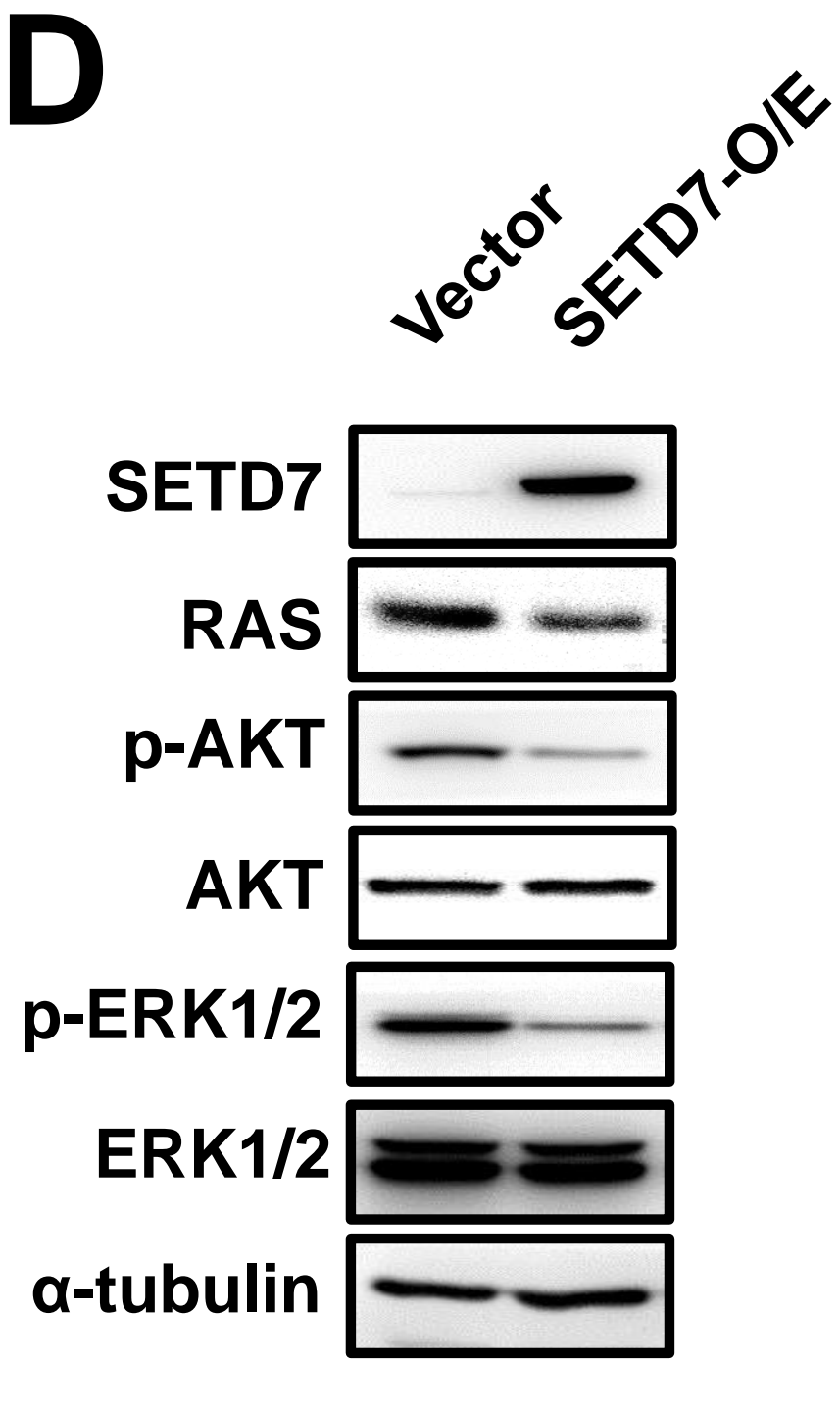

H

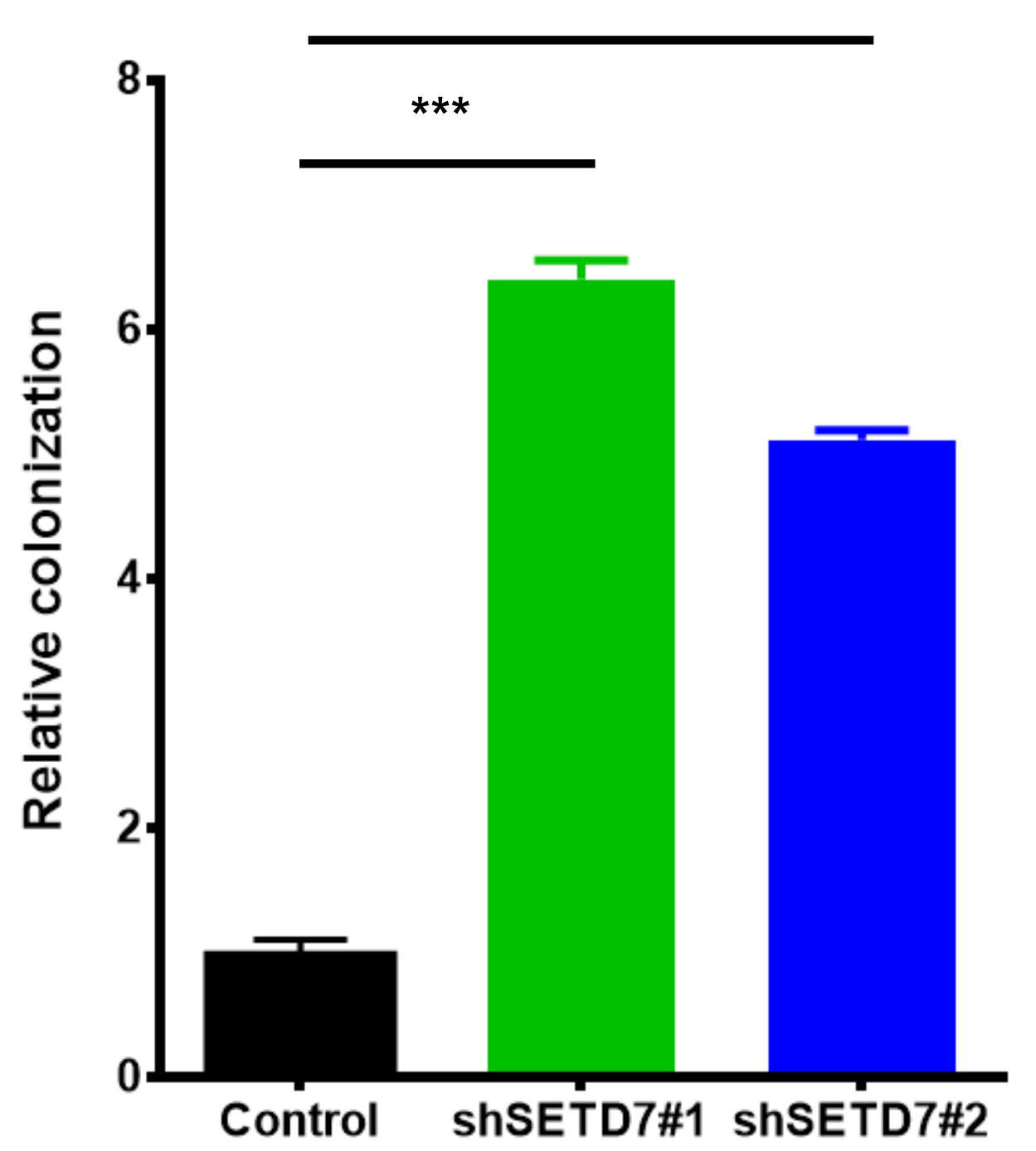

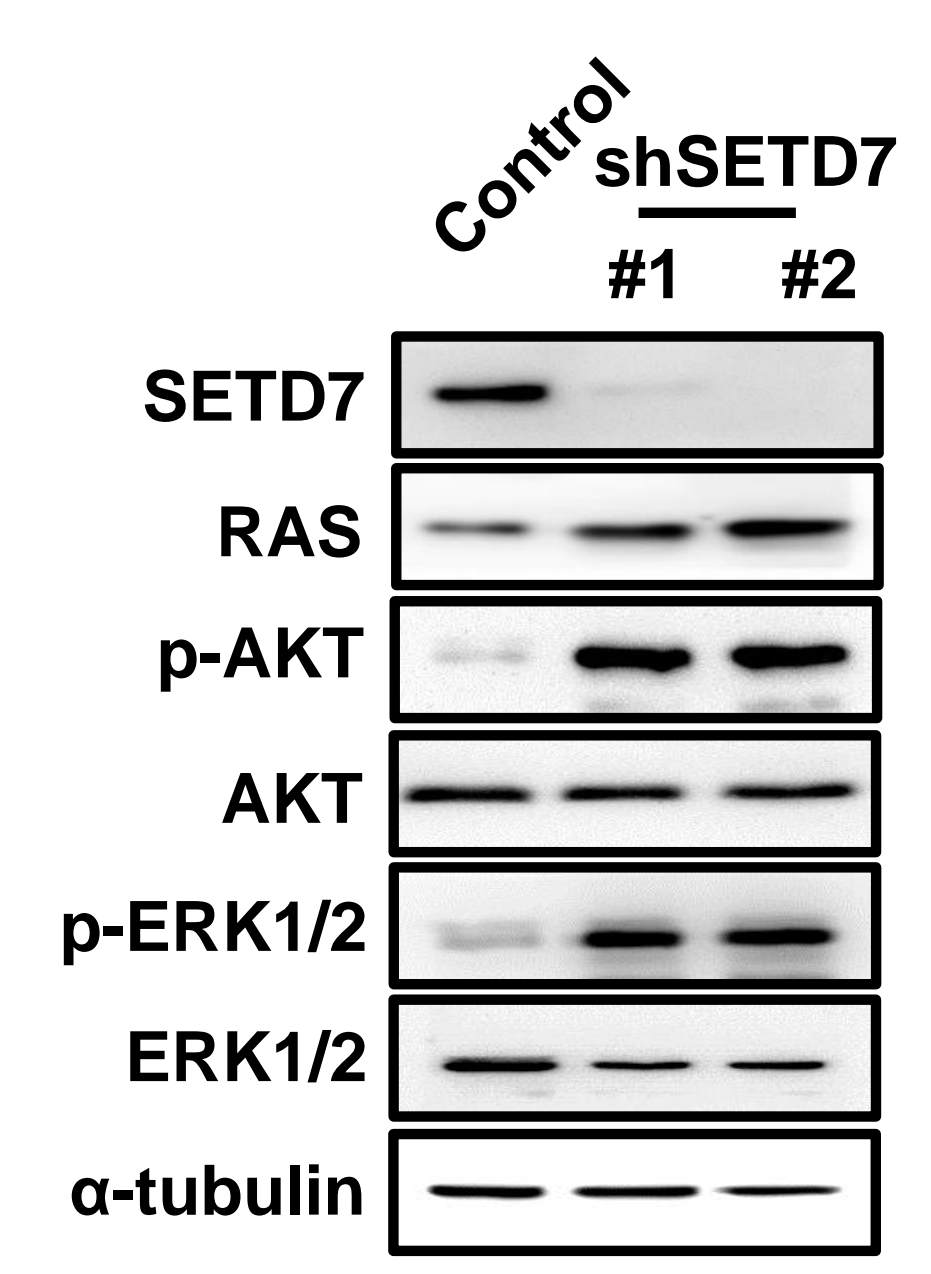




\section{Figure S2}

A

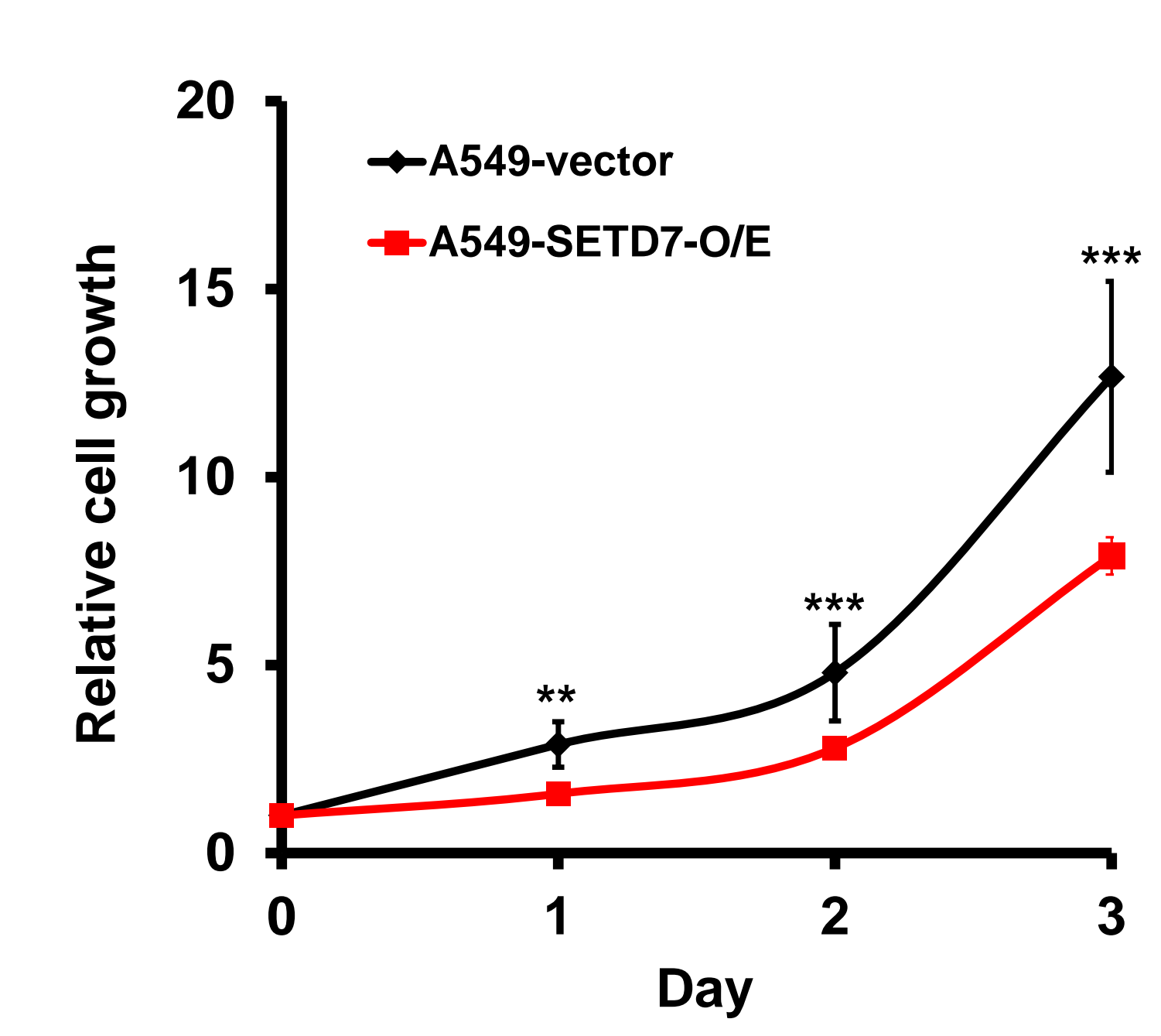

E

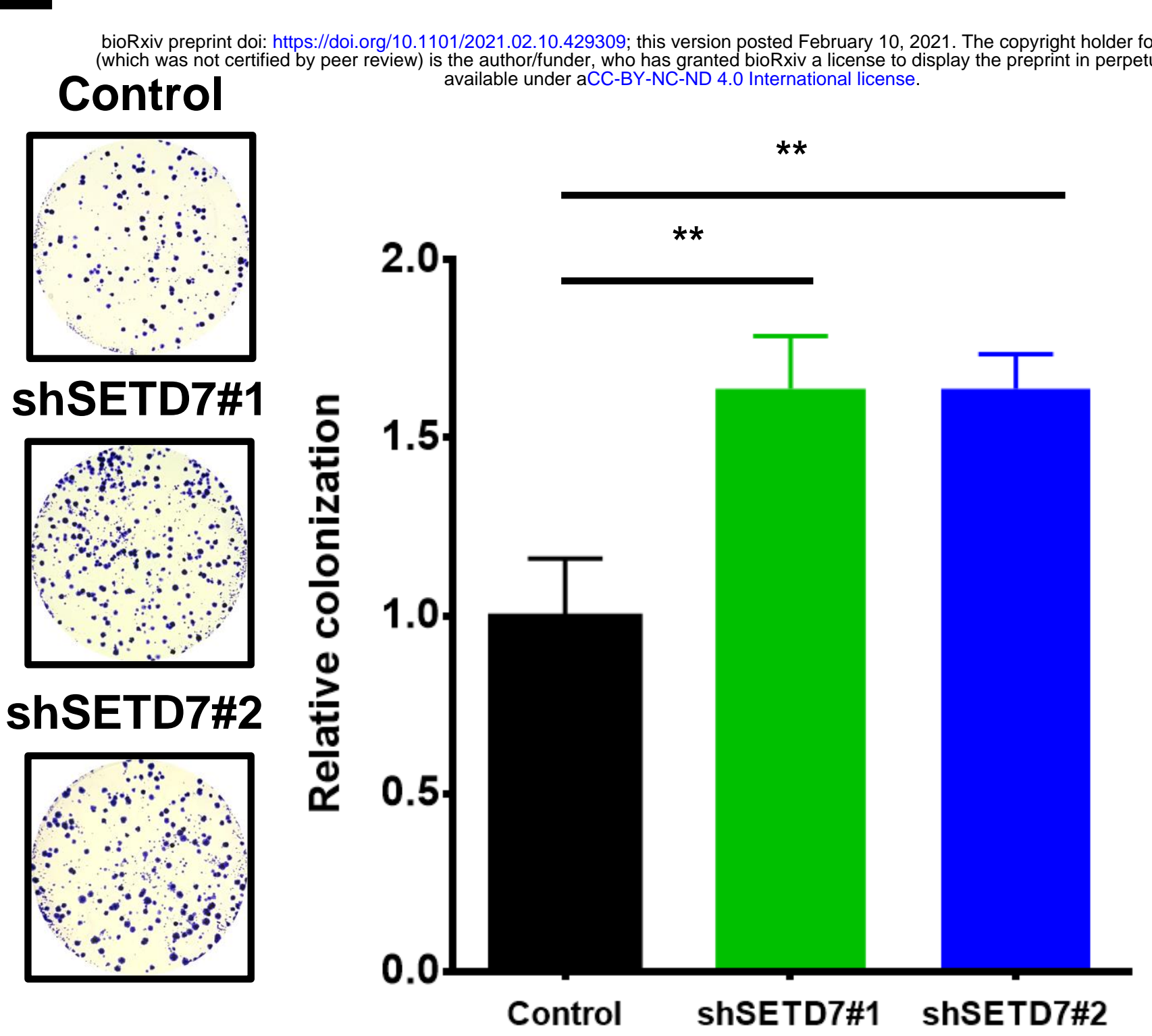

B
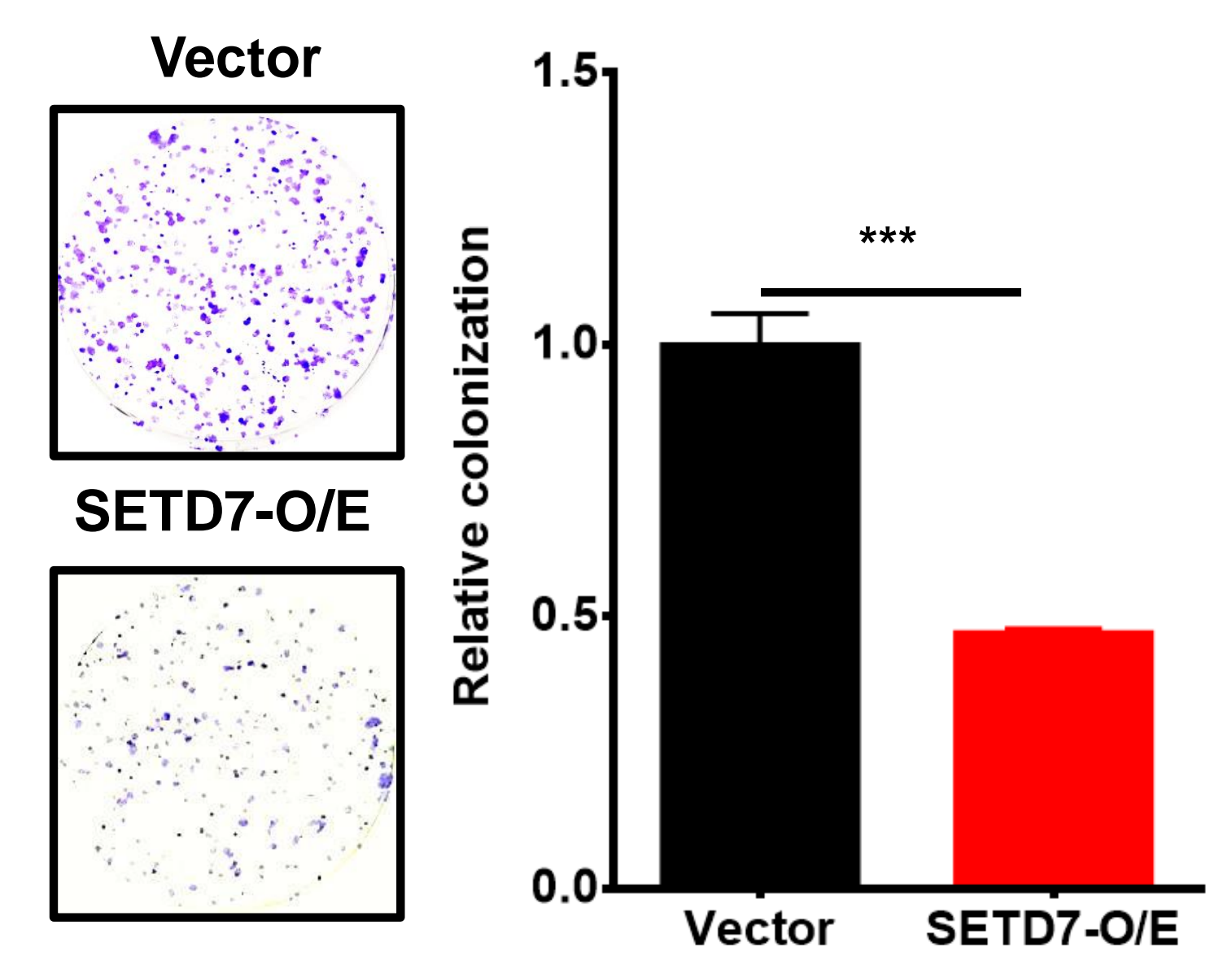

F
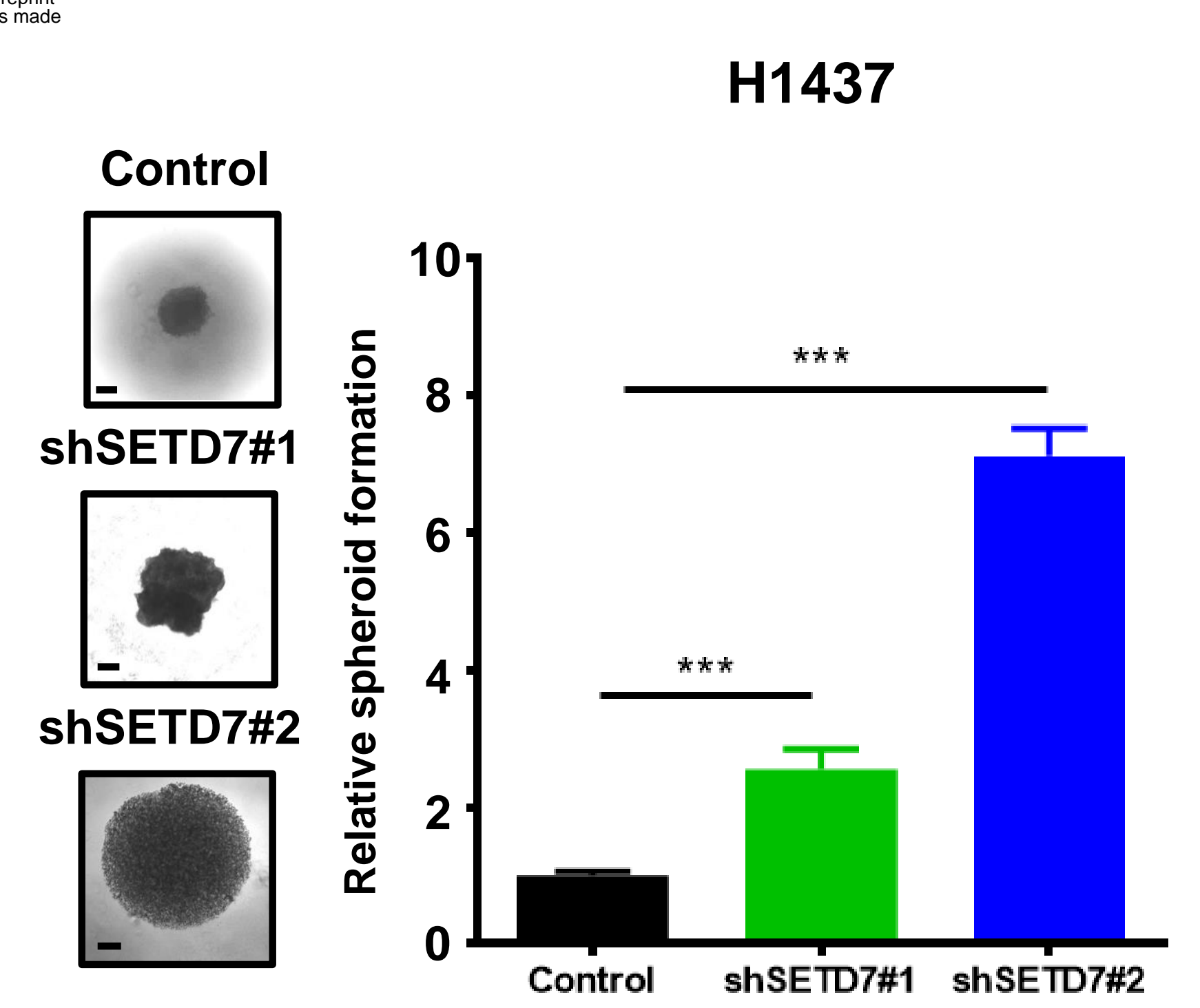

C

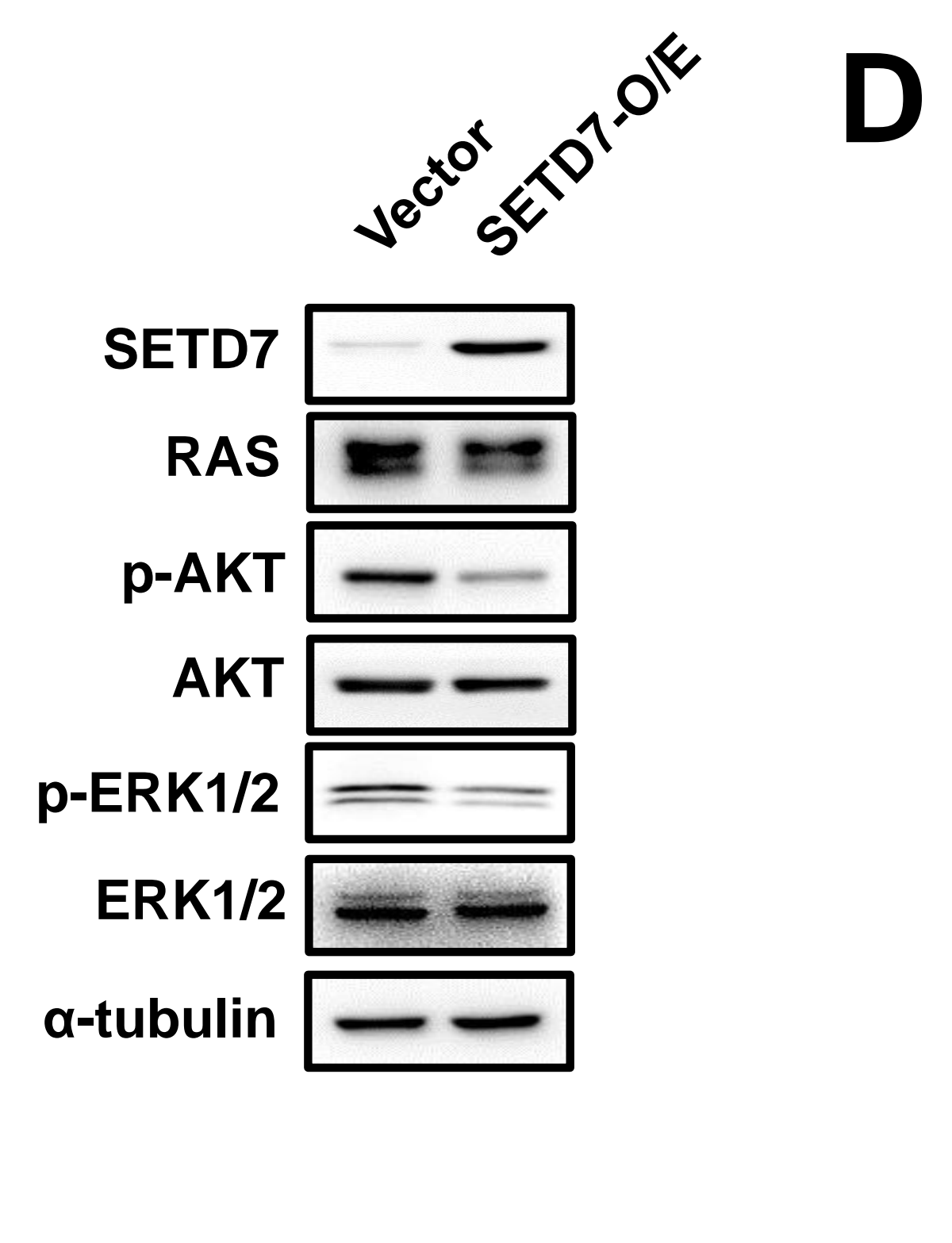

G

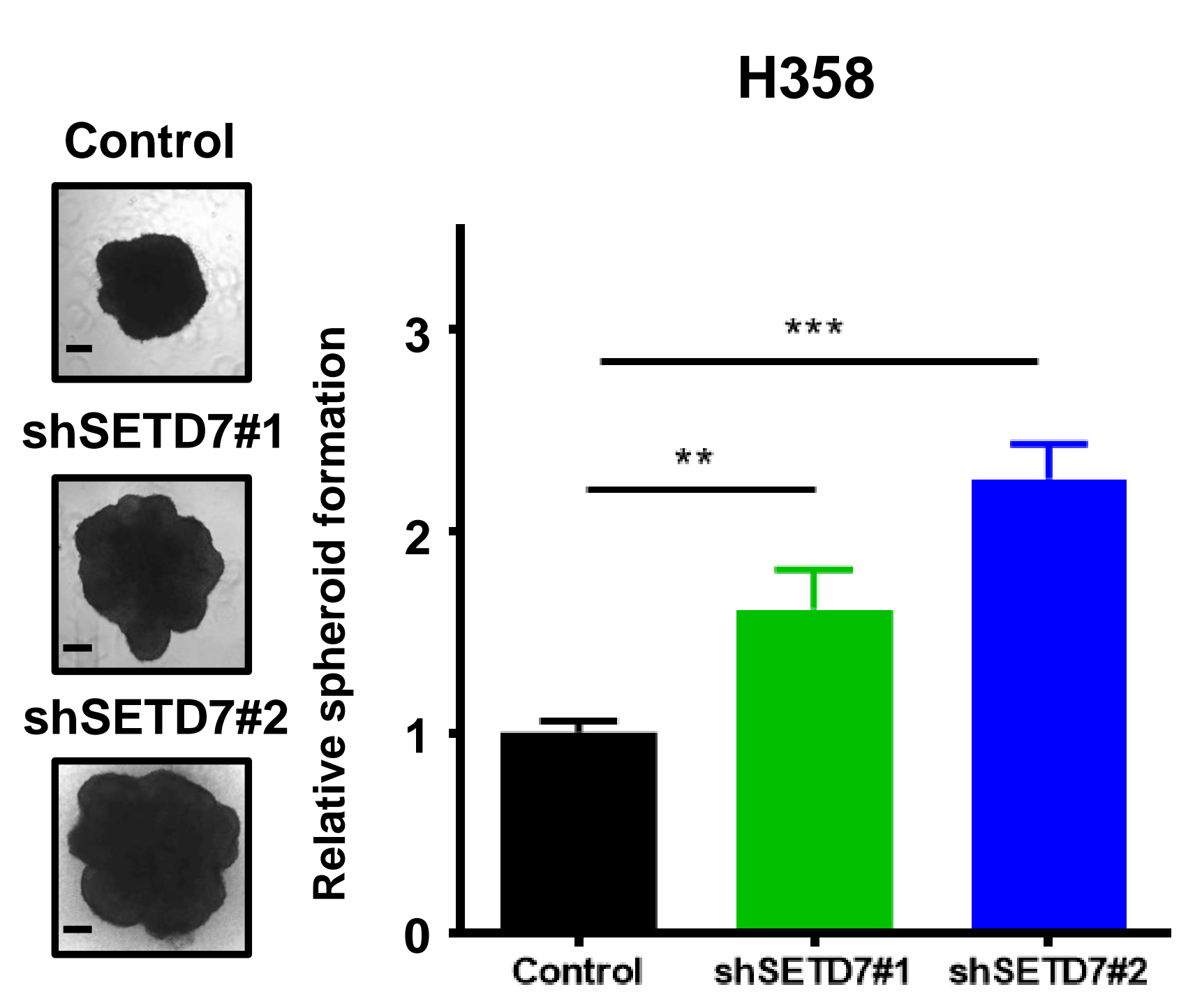

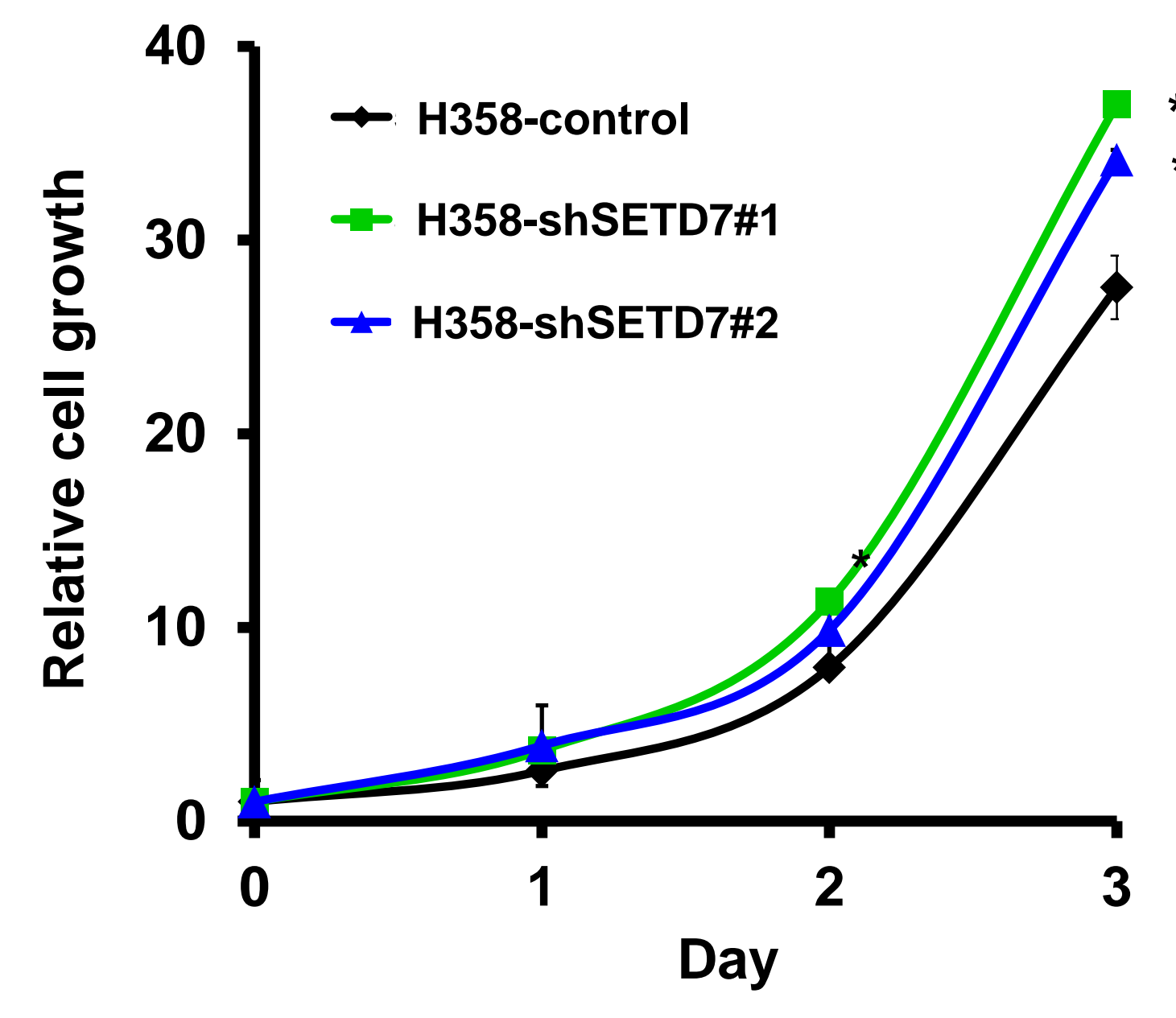

H

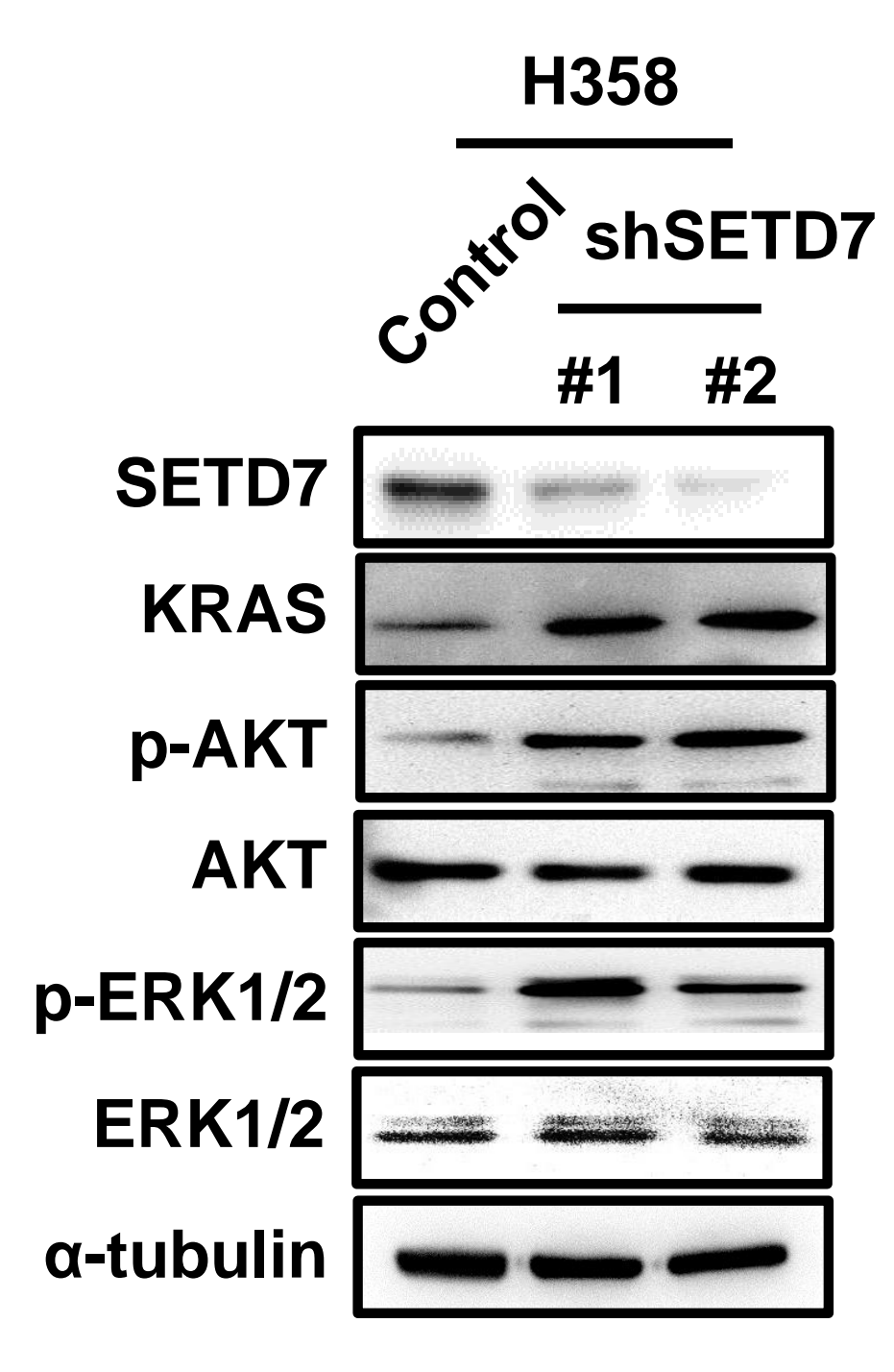




\section{Figure 3}

A

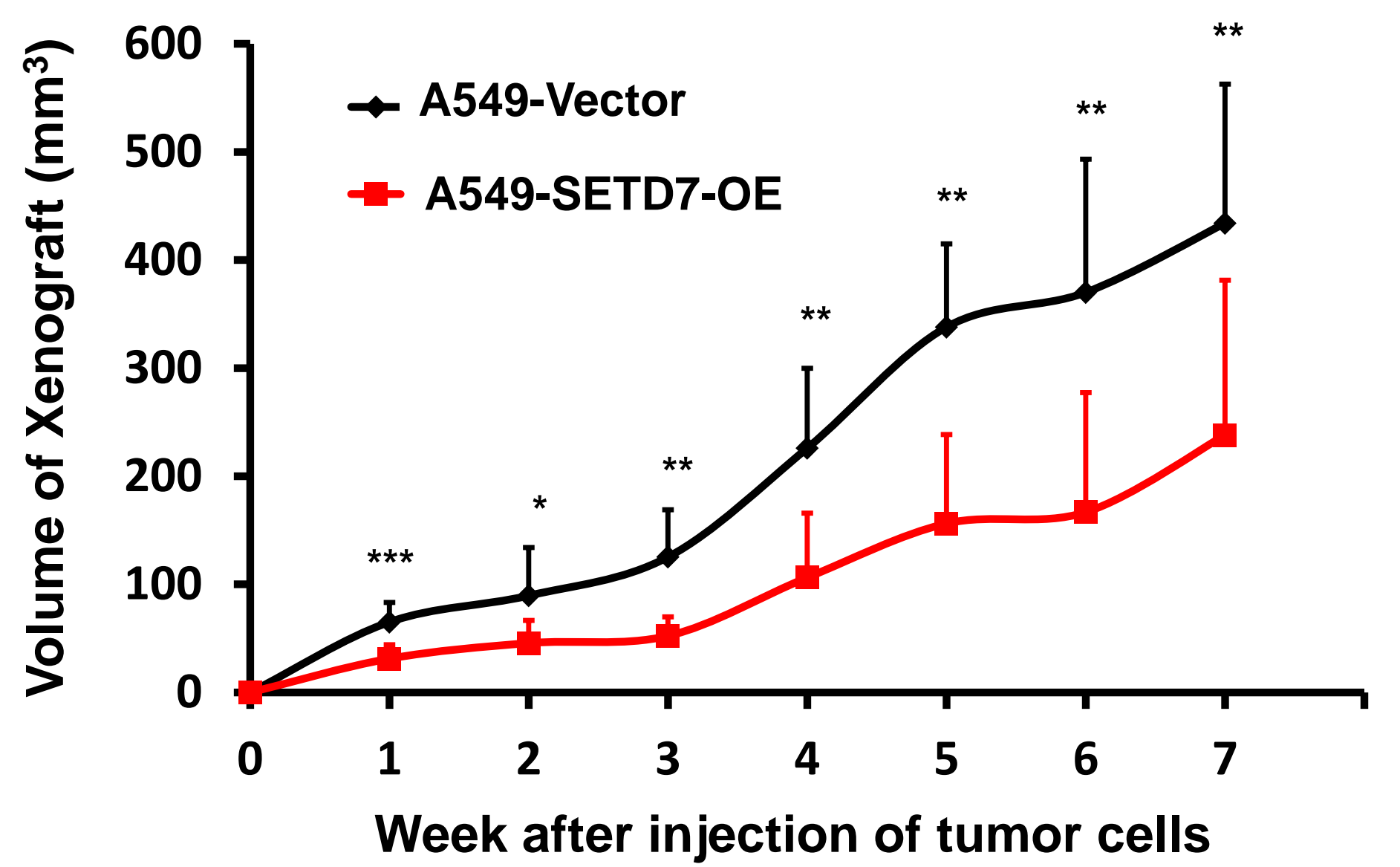

D

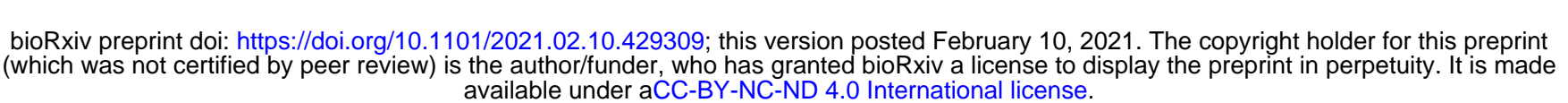

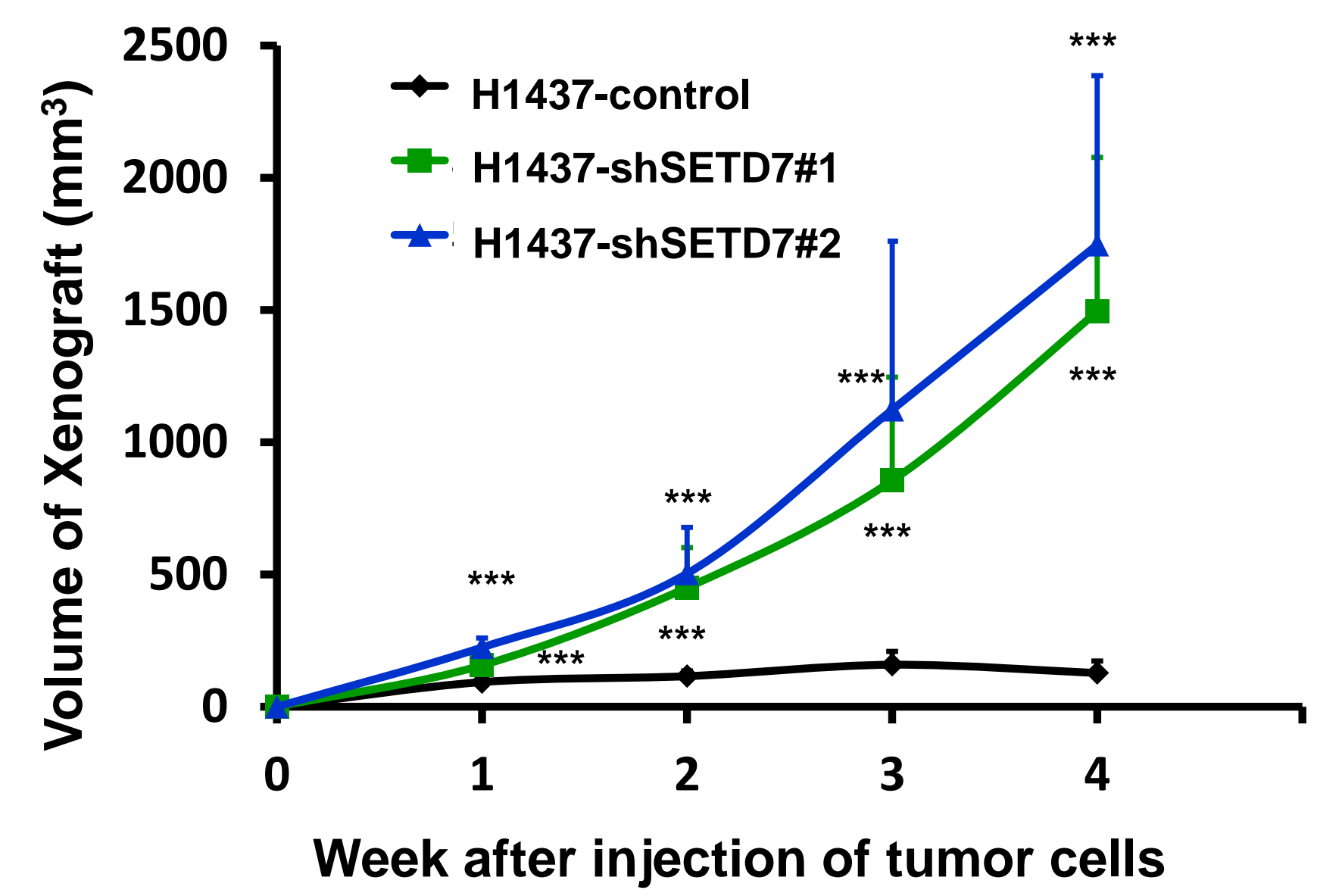

B
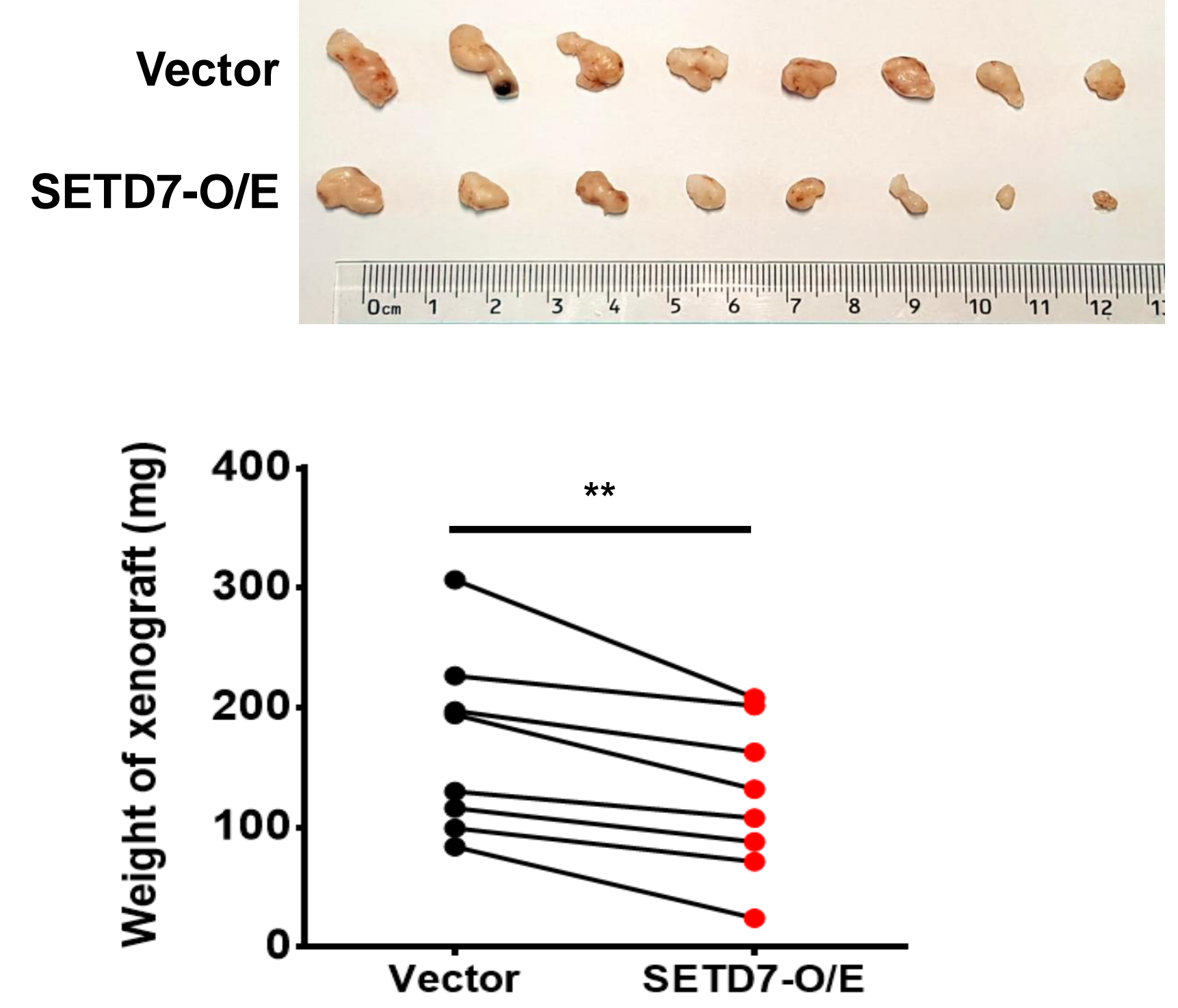

E
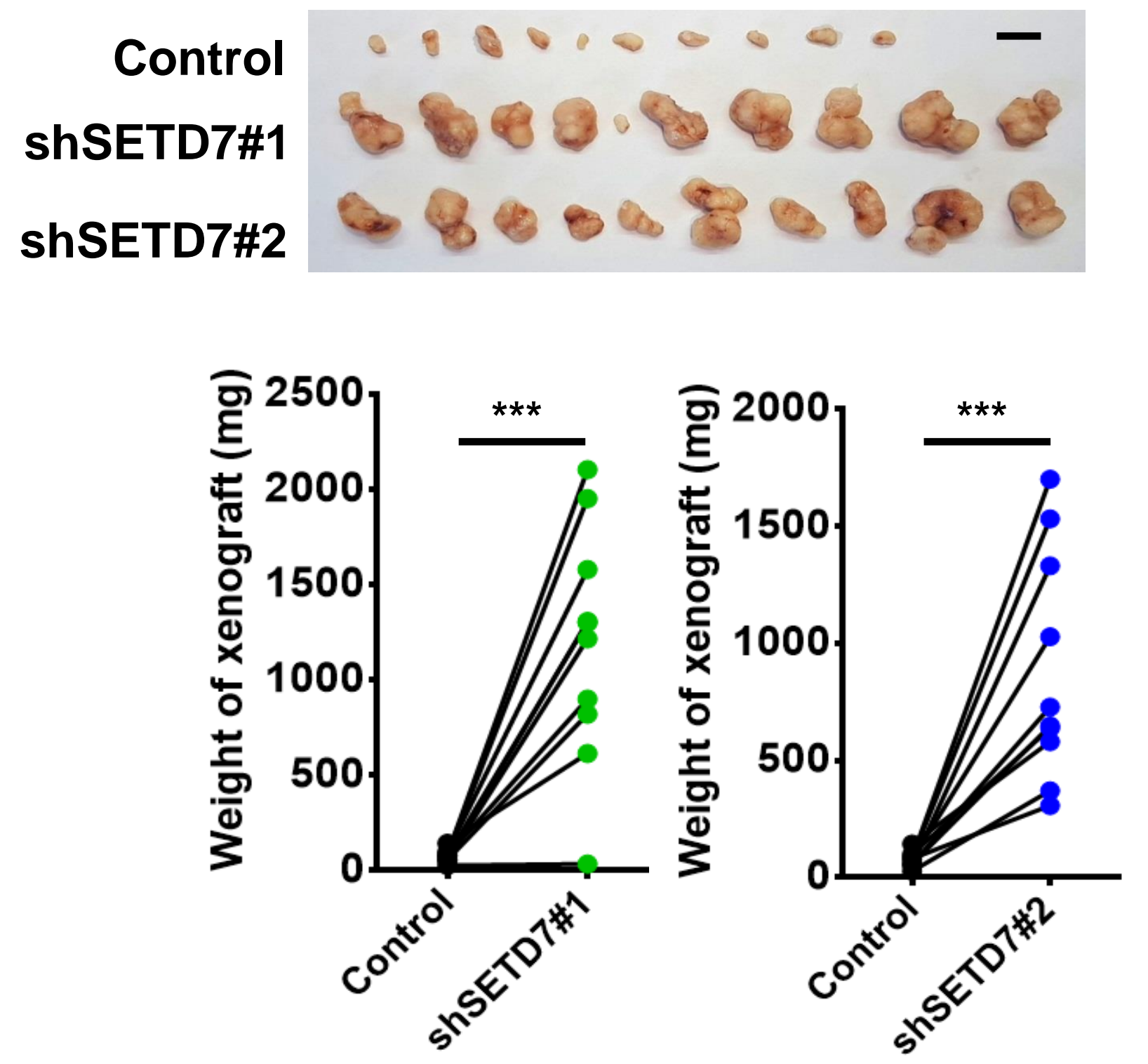

C

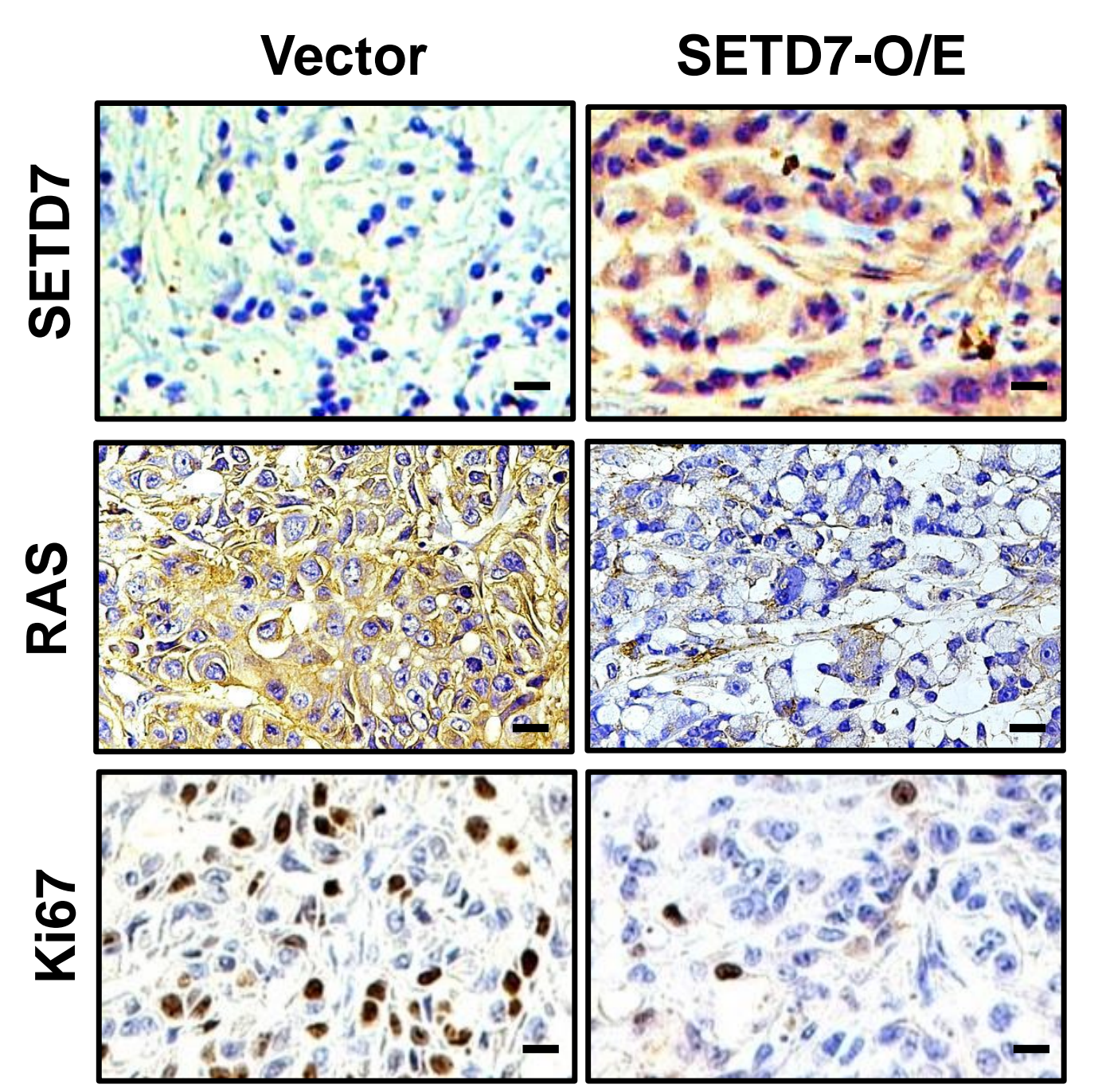

F

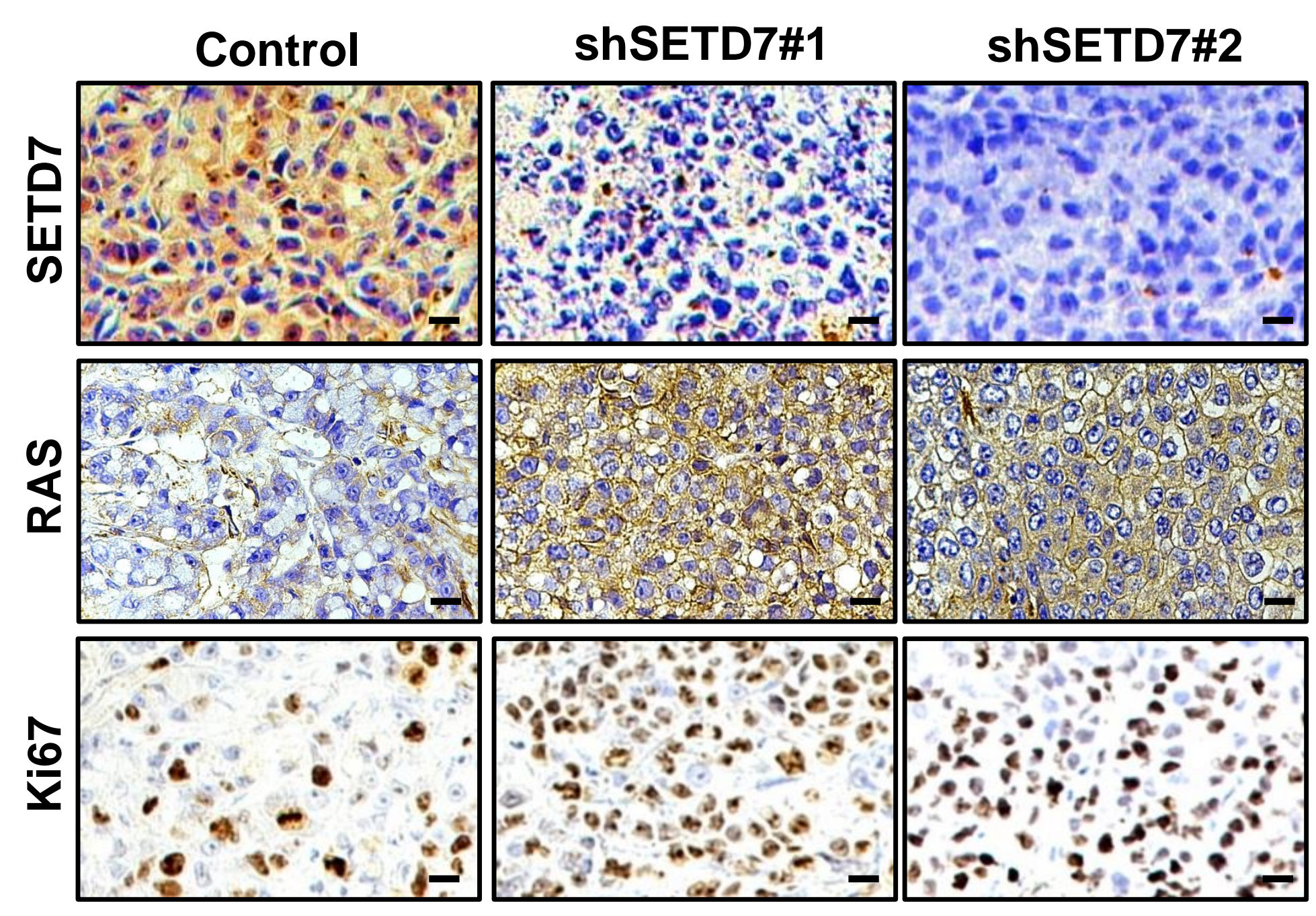




\section{Figure S3}

A

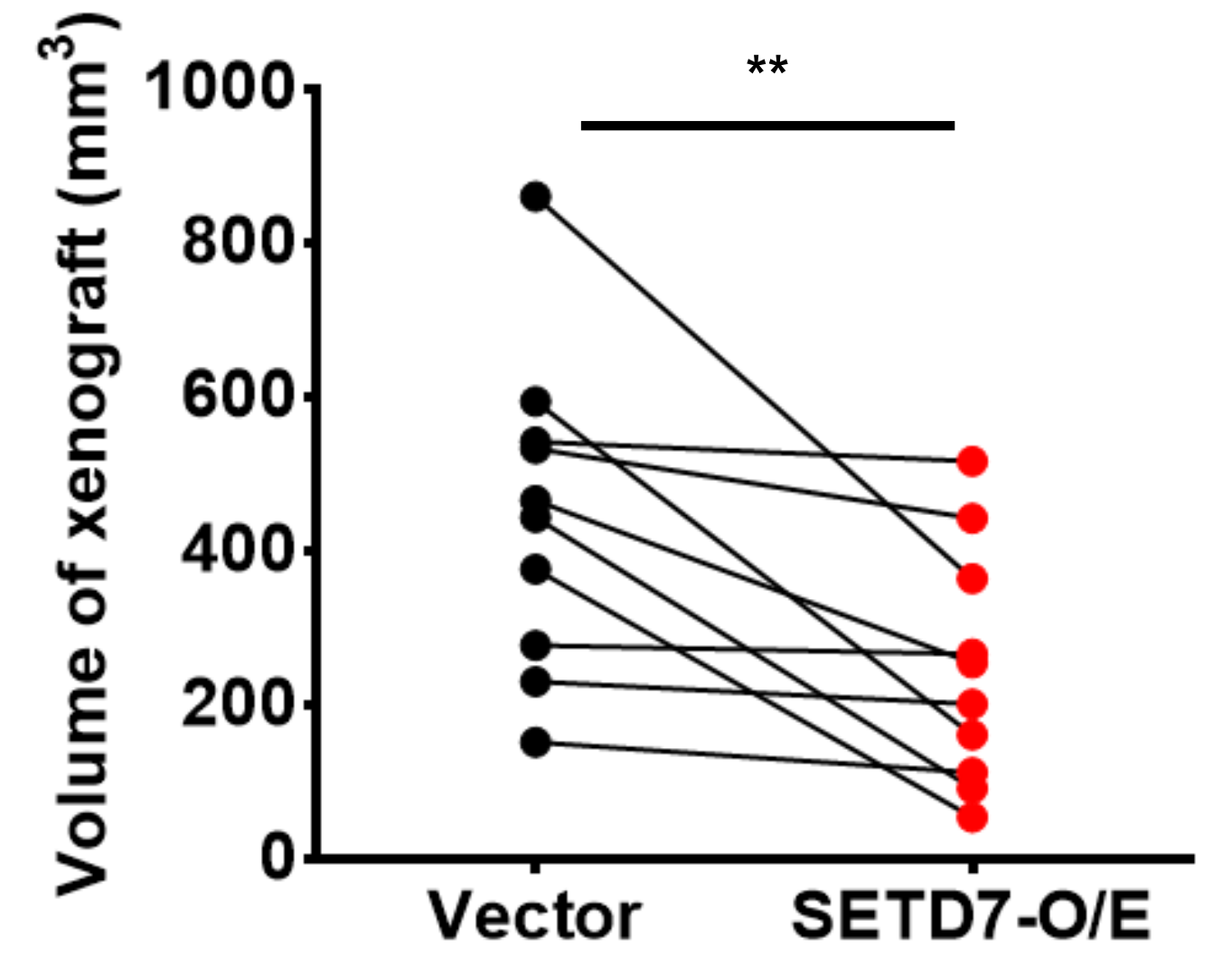

B

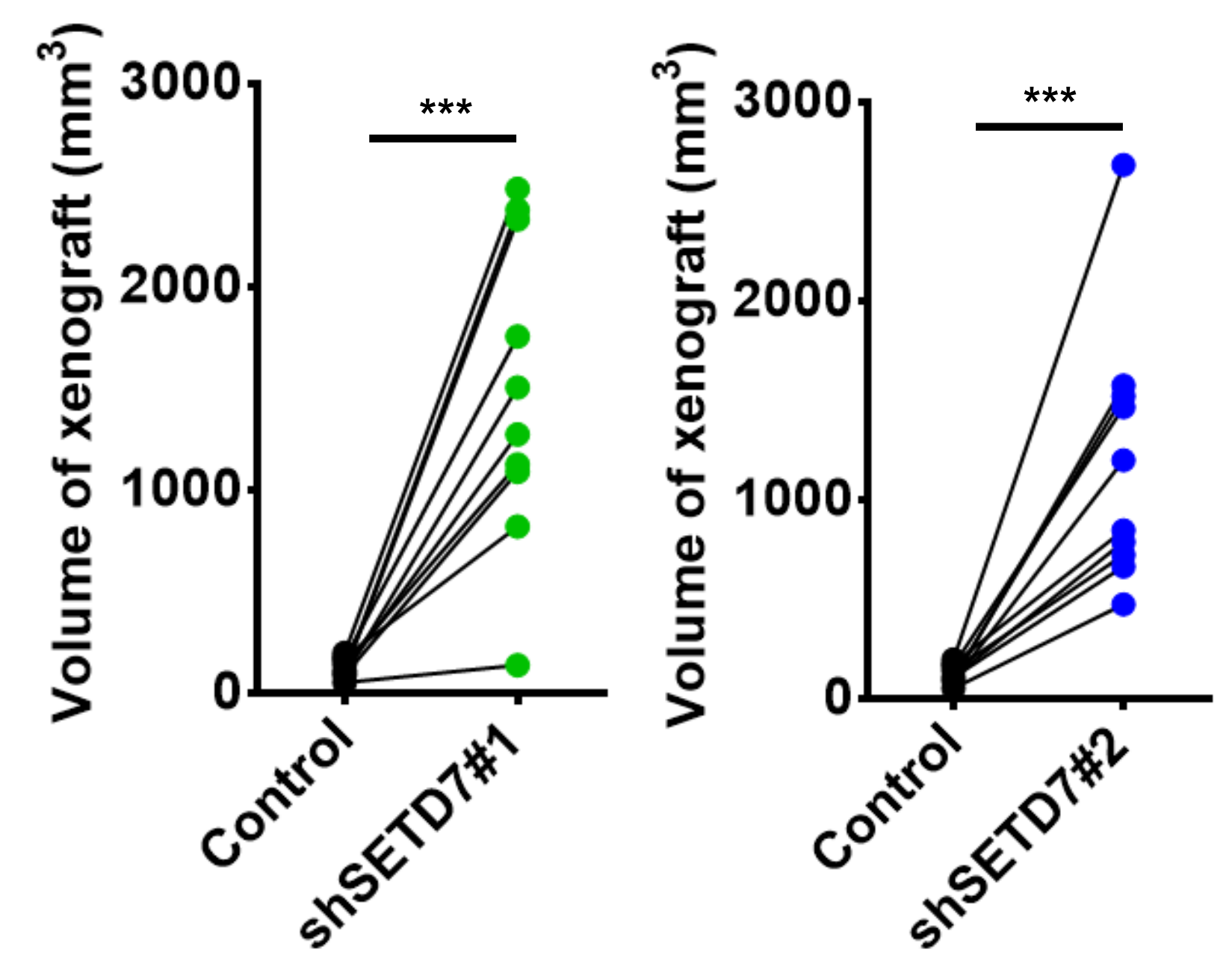


Figure 4

A

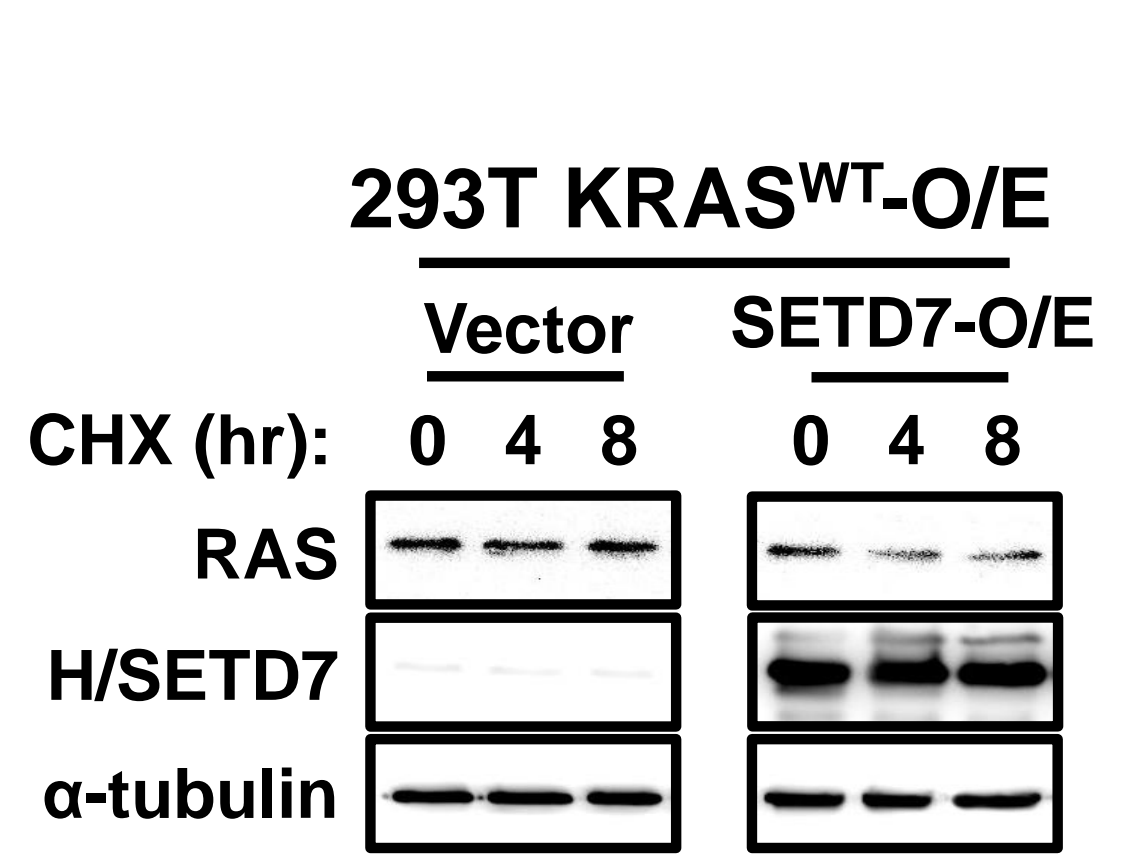

C
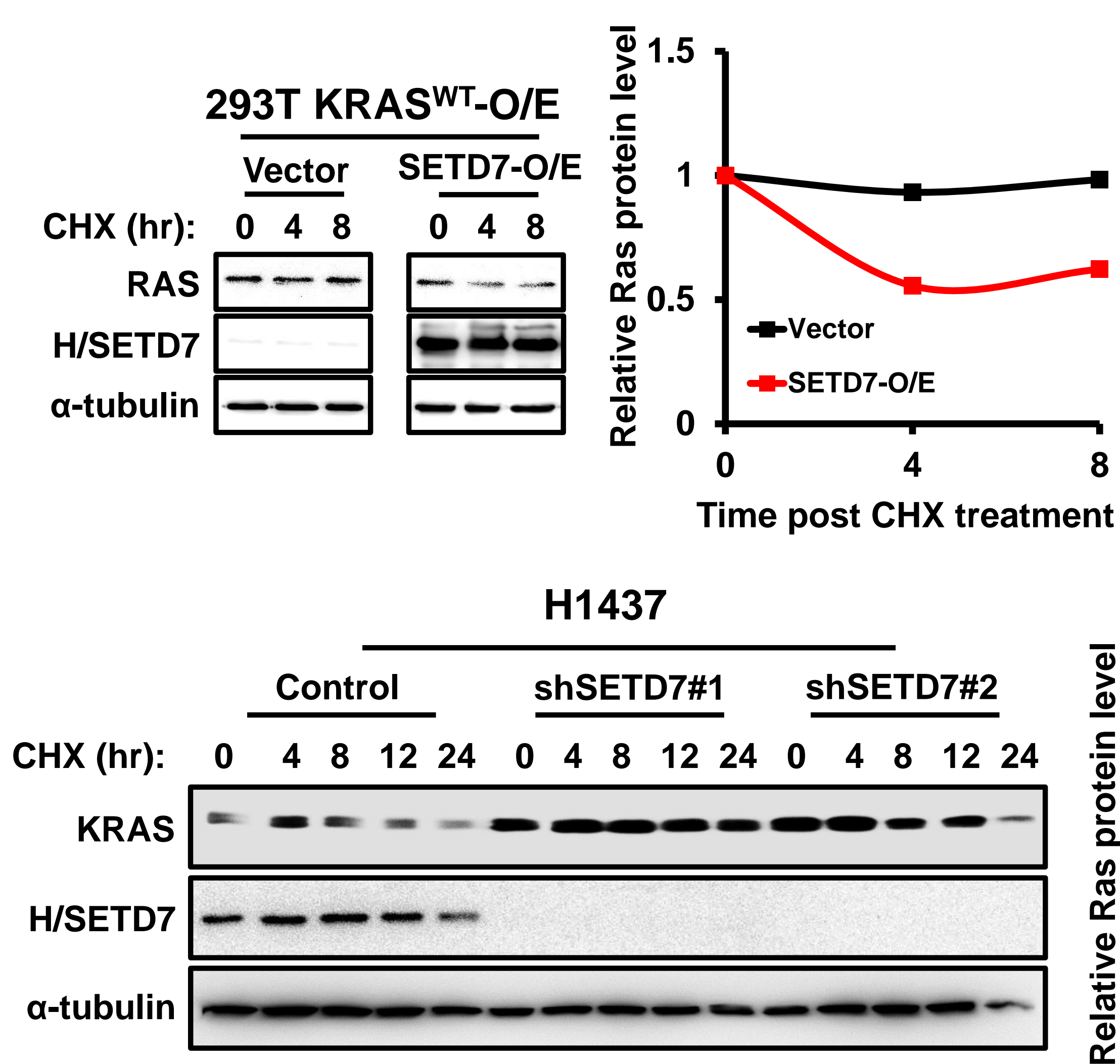

Time post $\mathrm{CHX}$ treatment (hr)

B

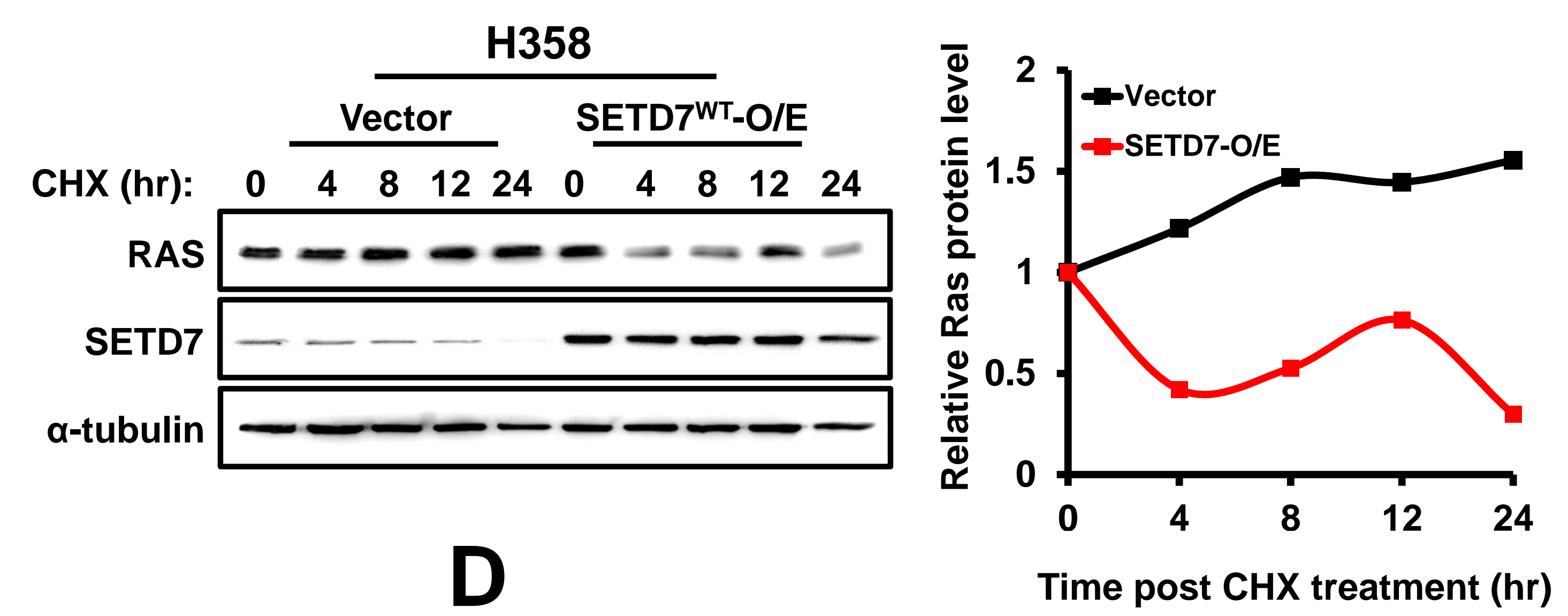

H358-
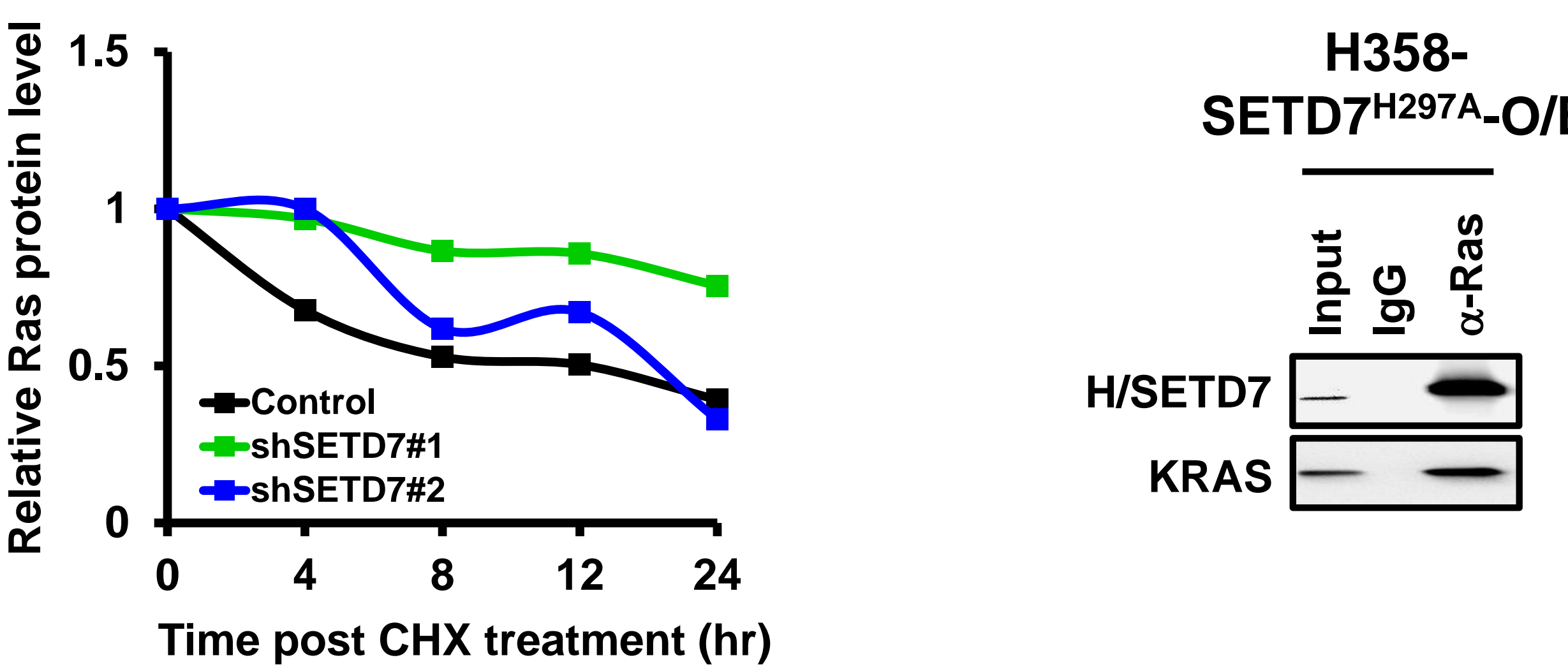

E

$\mathbf{F}$
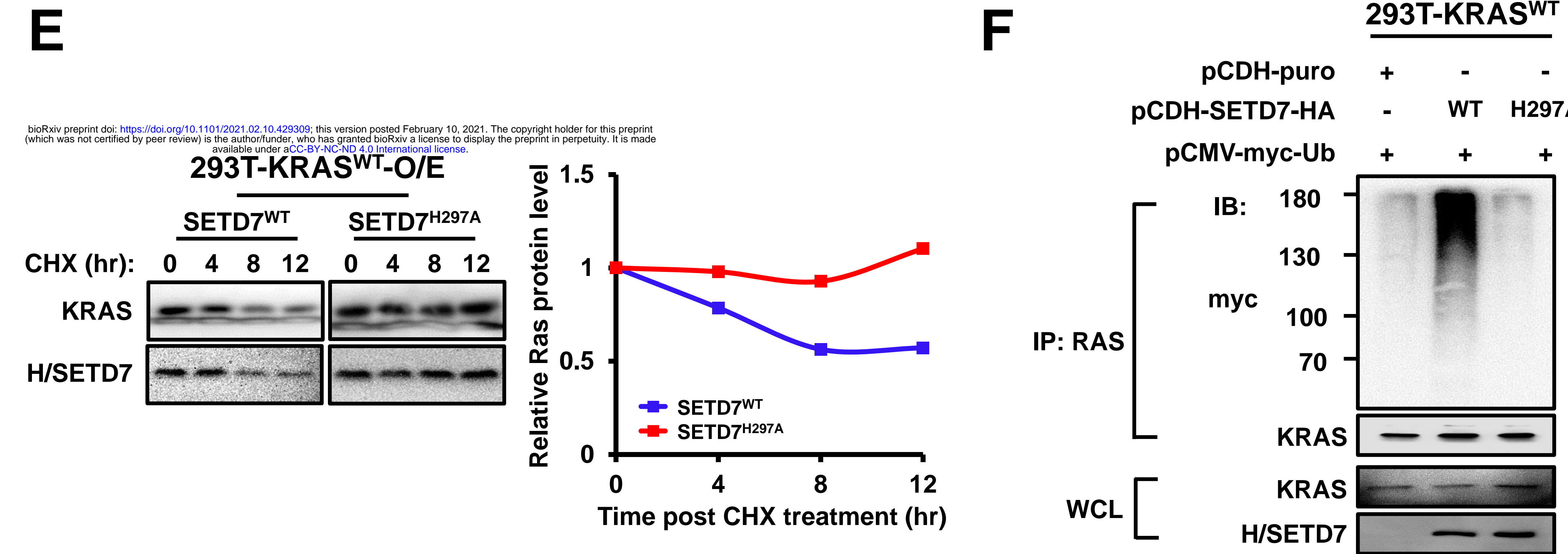

293T-KRASWT

pCDH-puro + - -

G pCDH-SETD7-HA - WT H297A

H

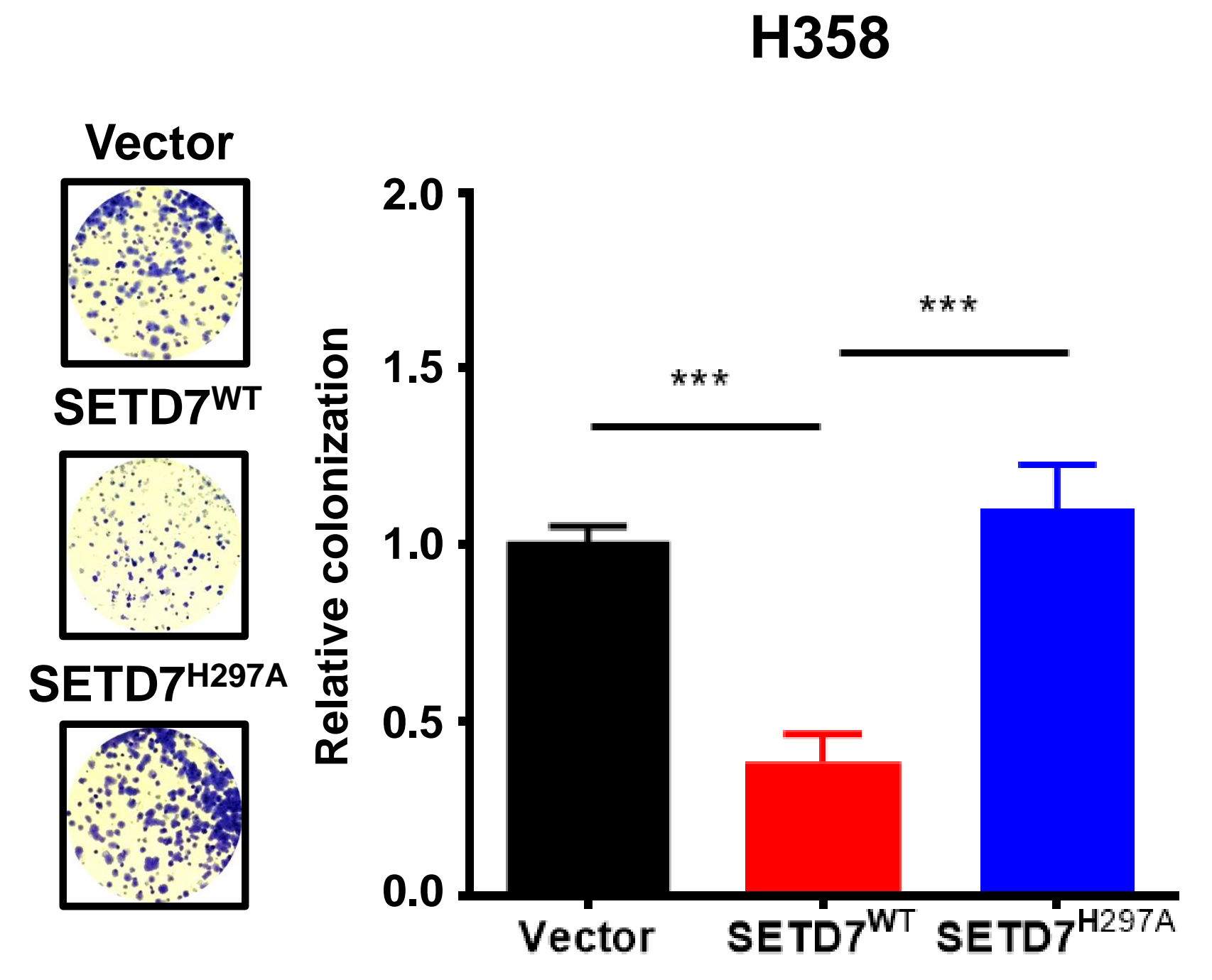

I

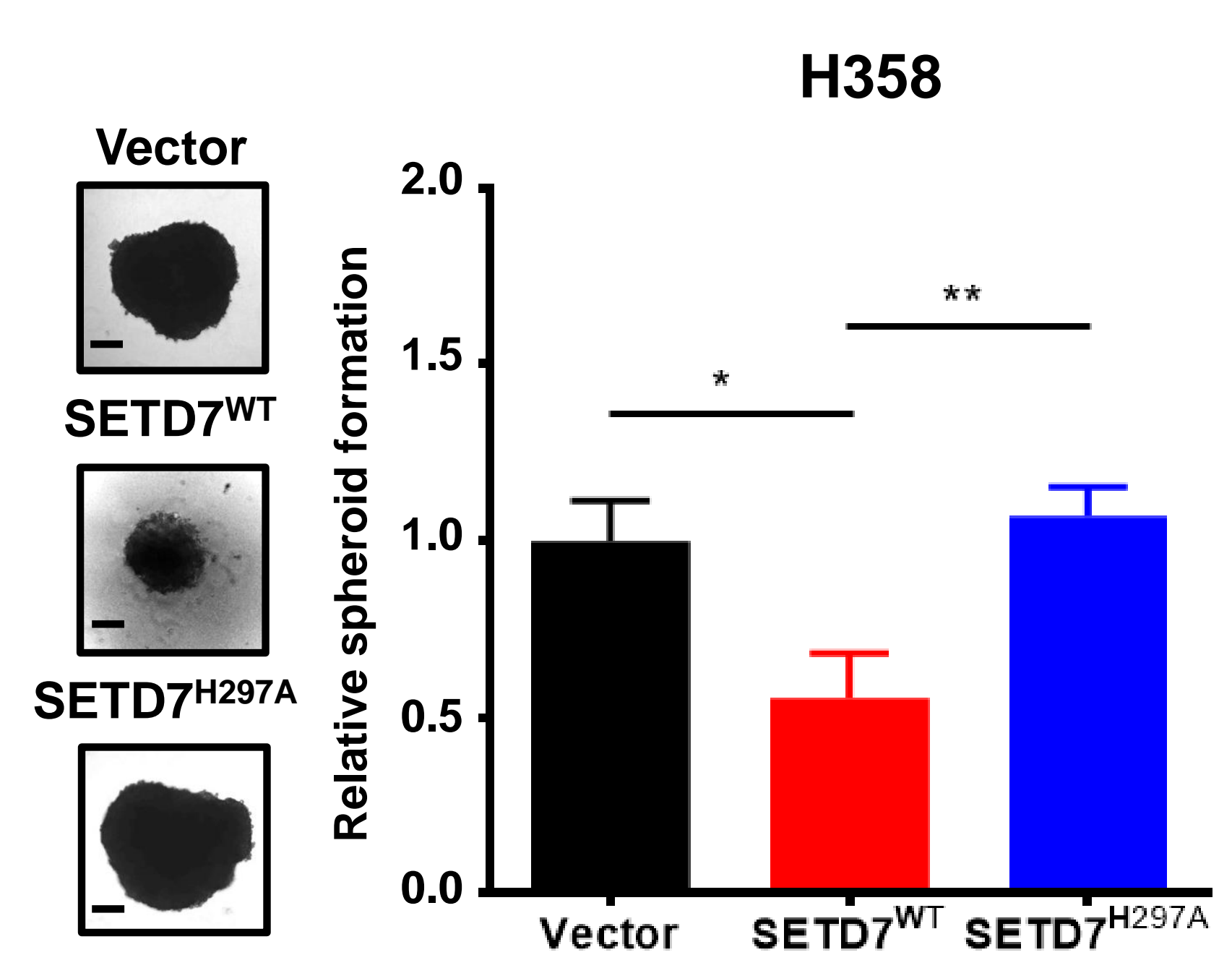

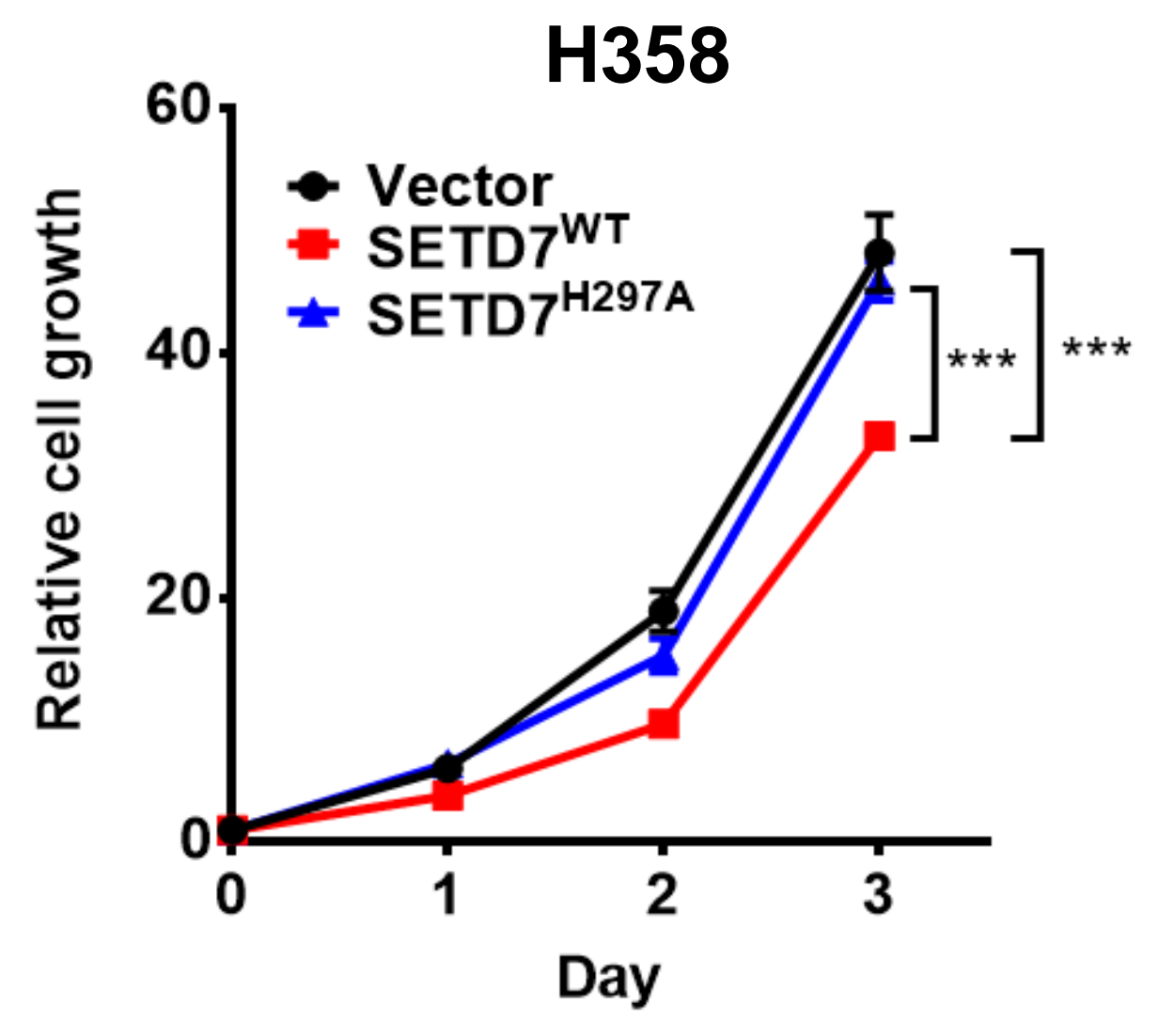

J

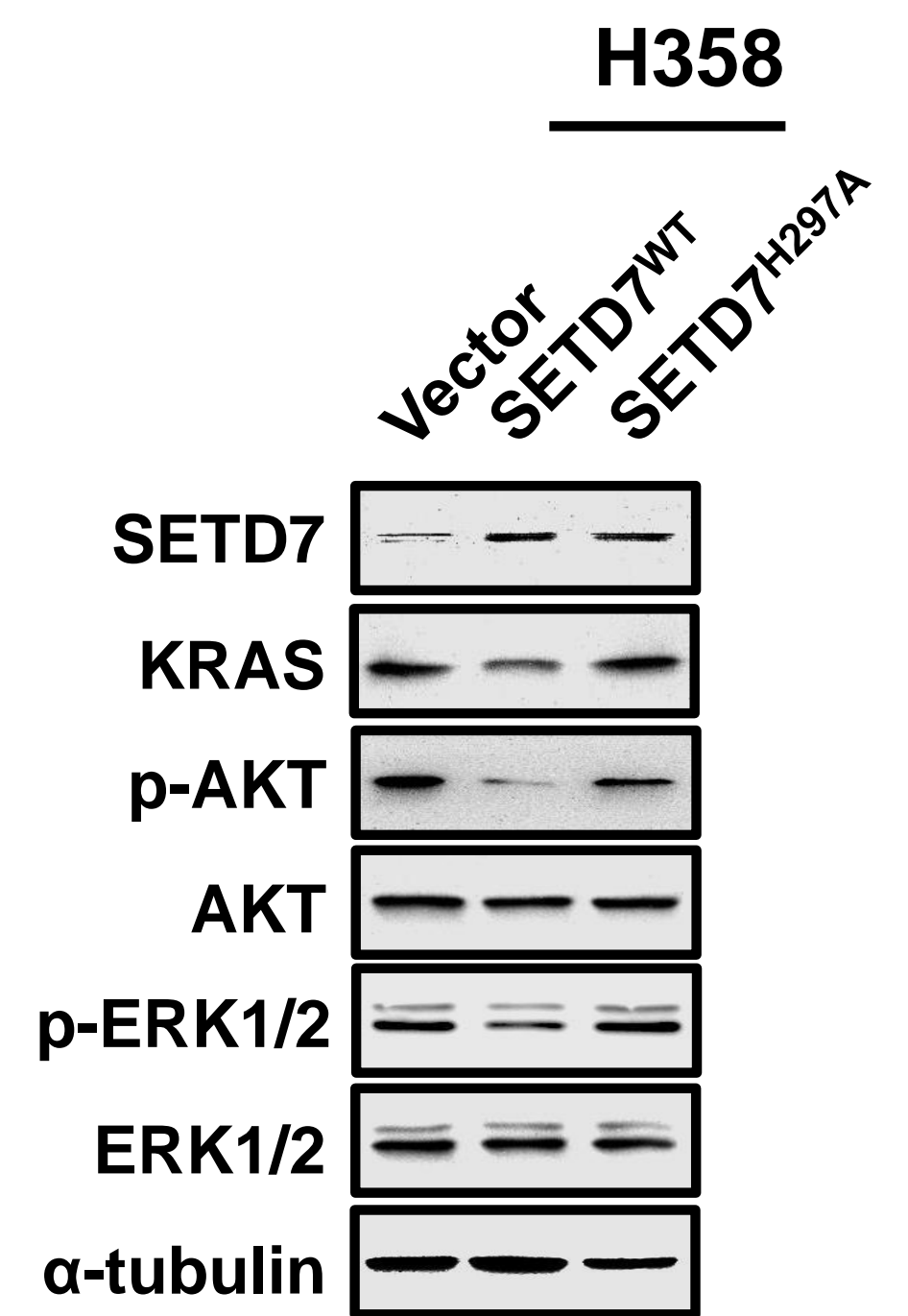




\section{Figure S4}

A

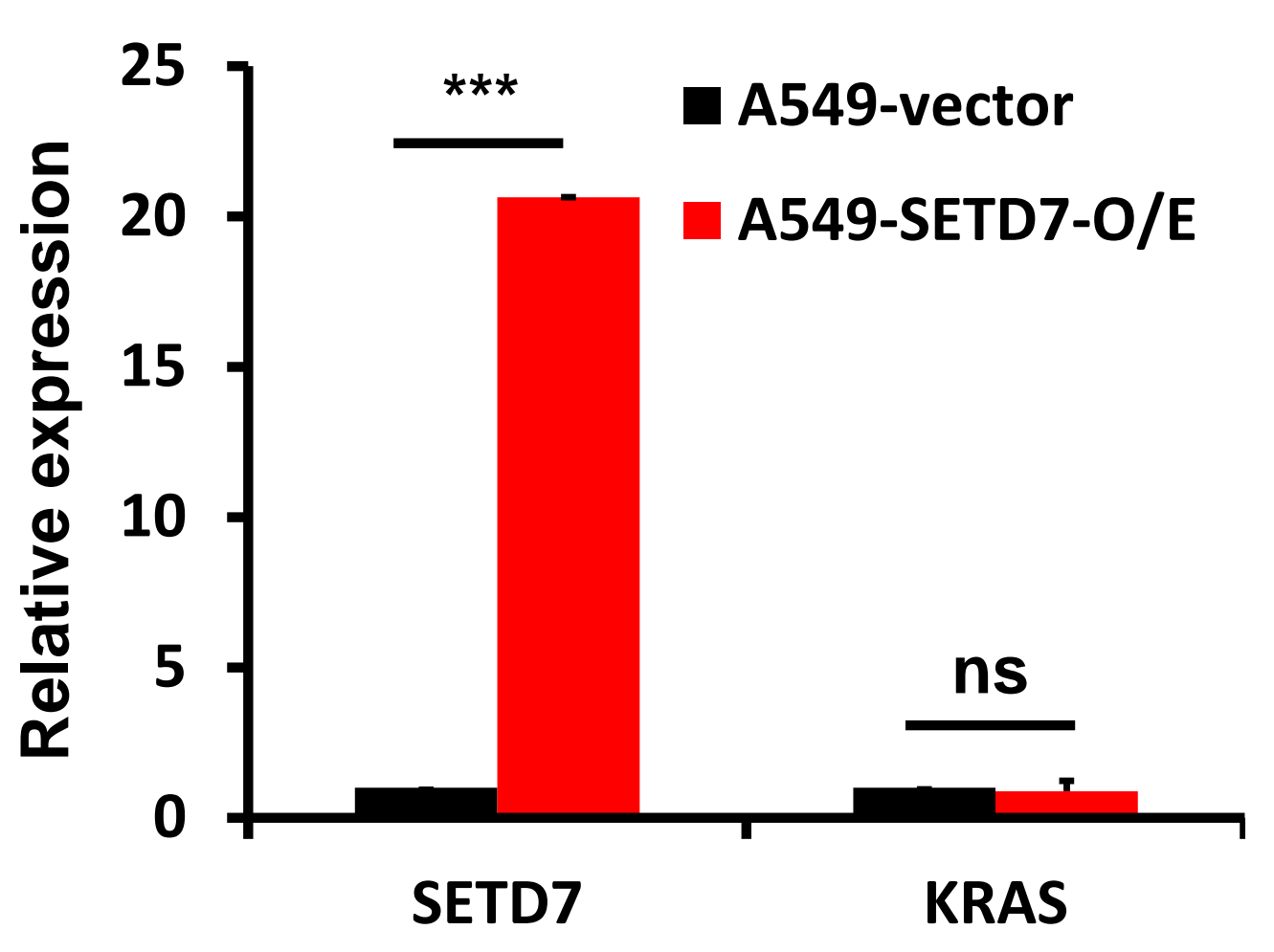

D

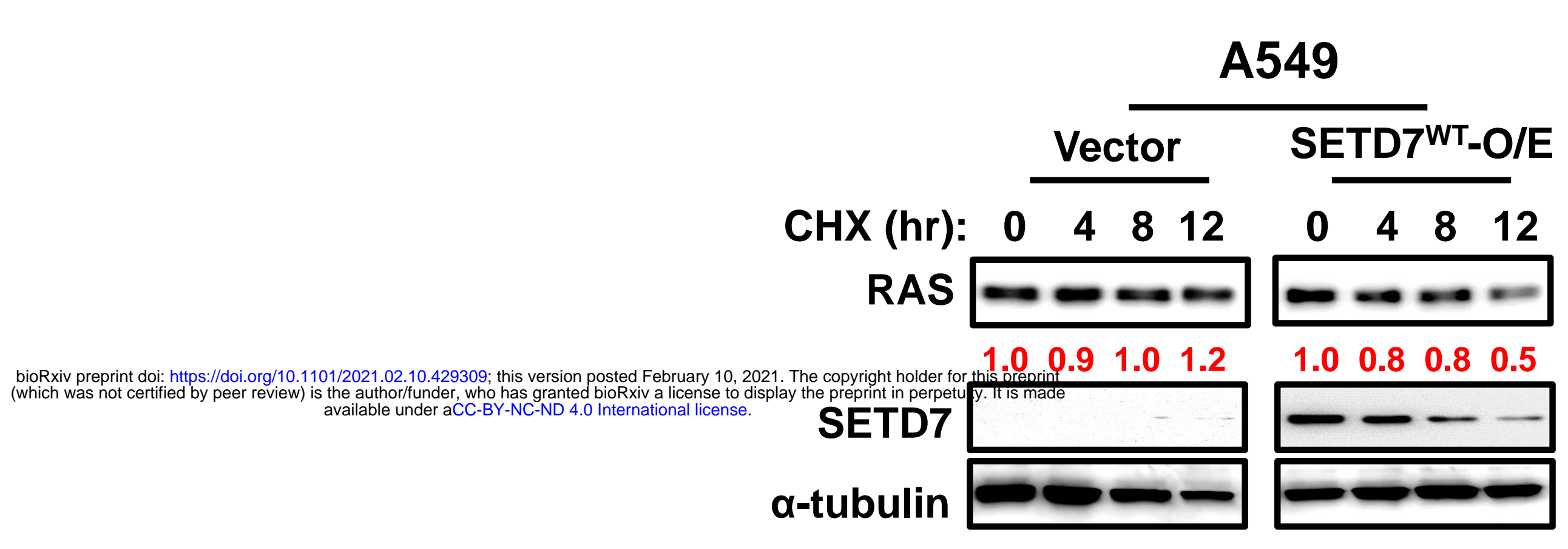

F
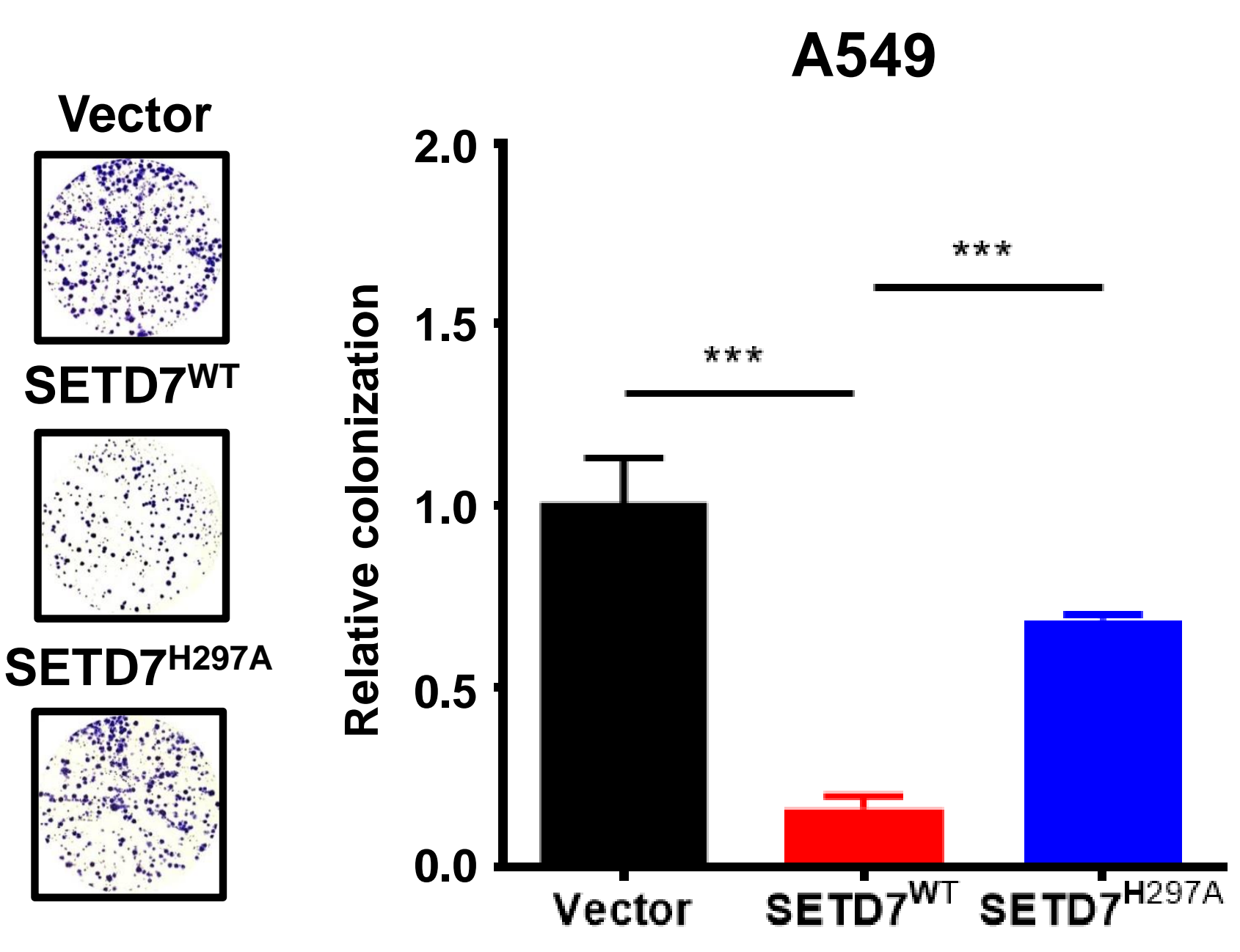

G
B

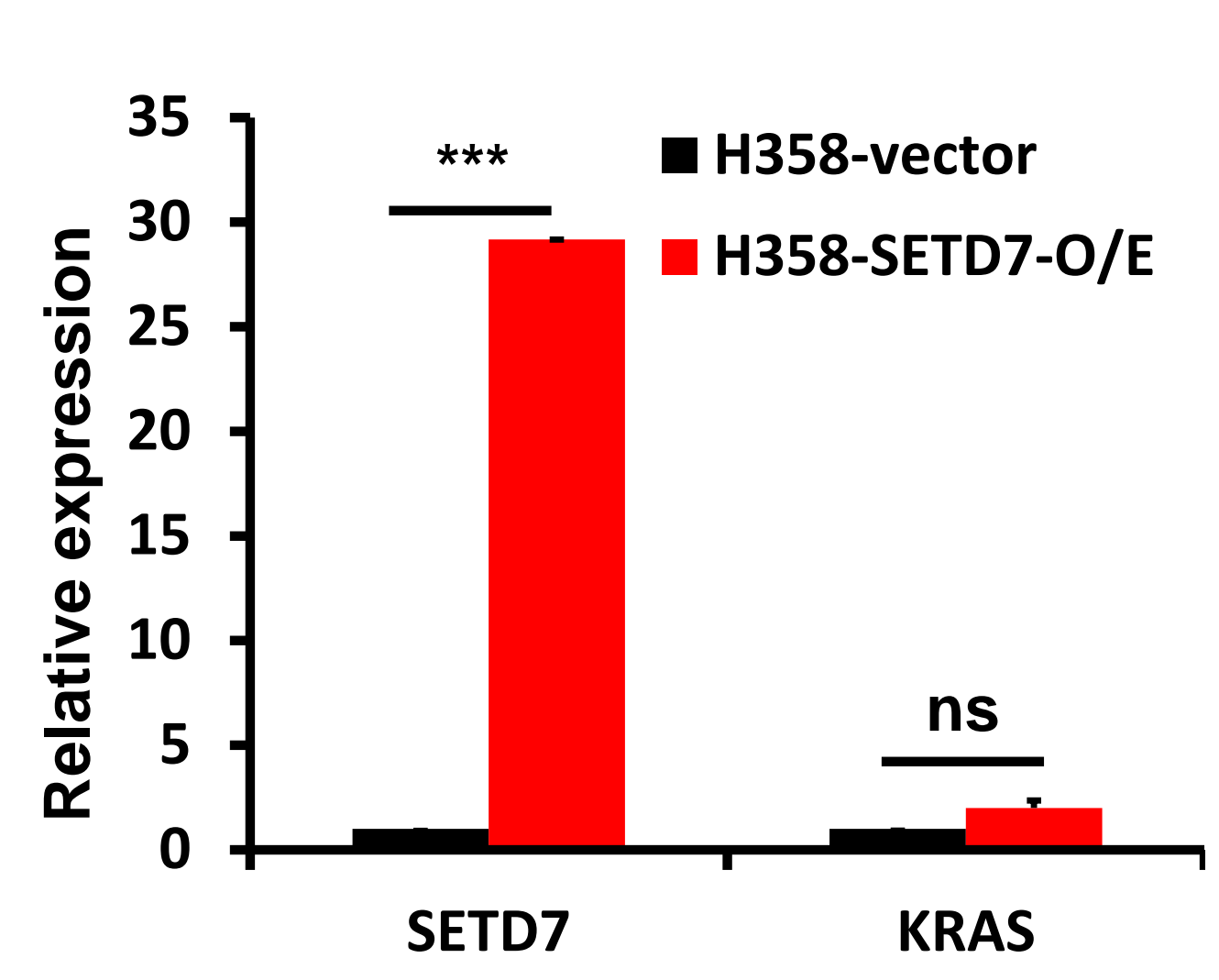

E
C

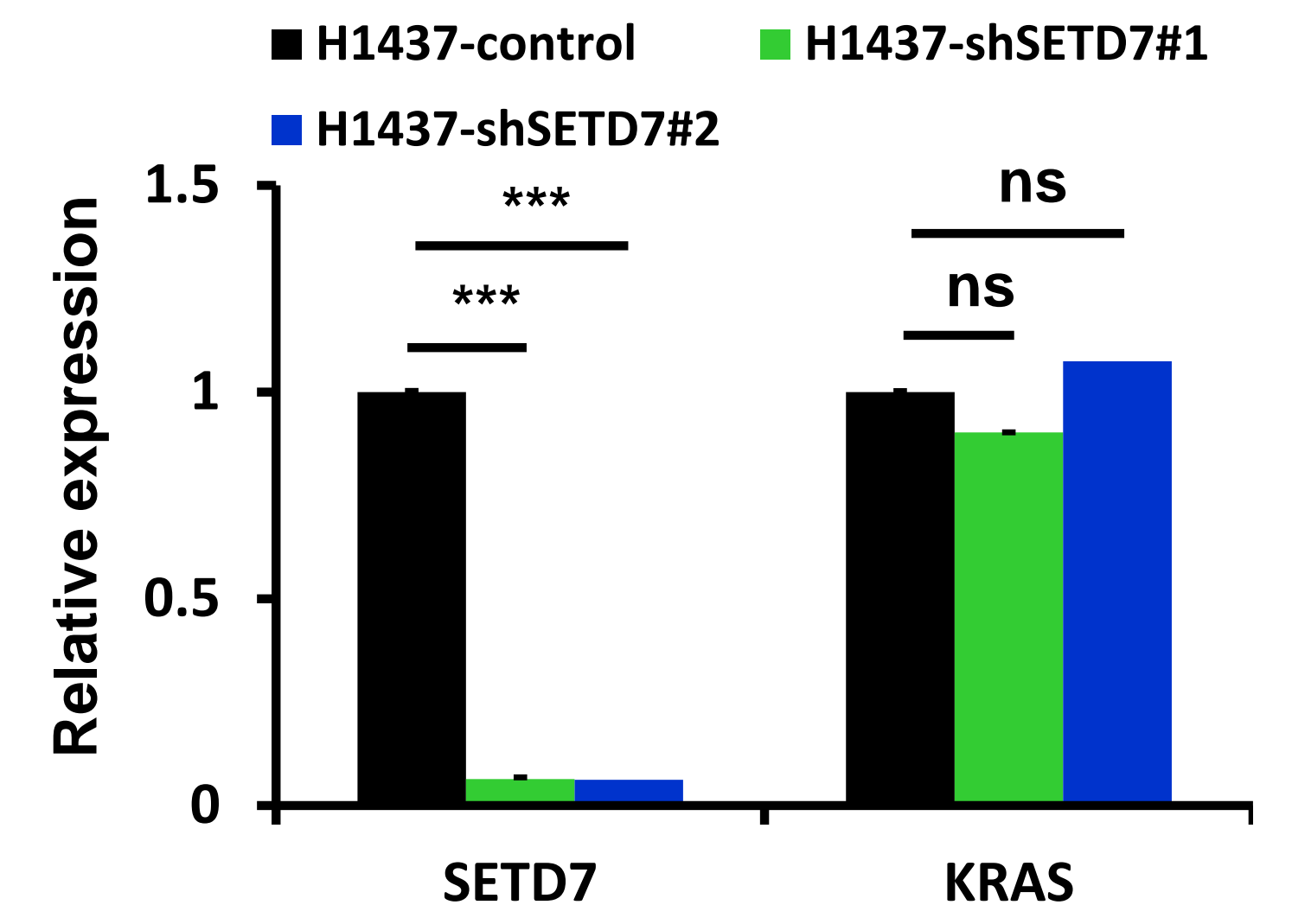

A549

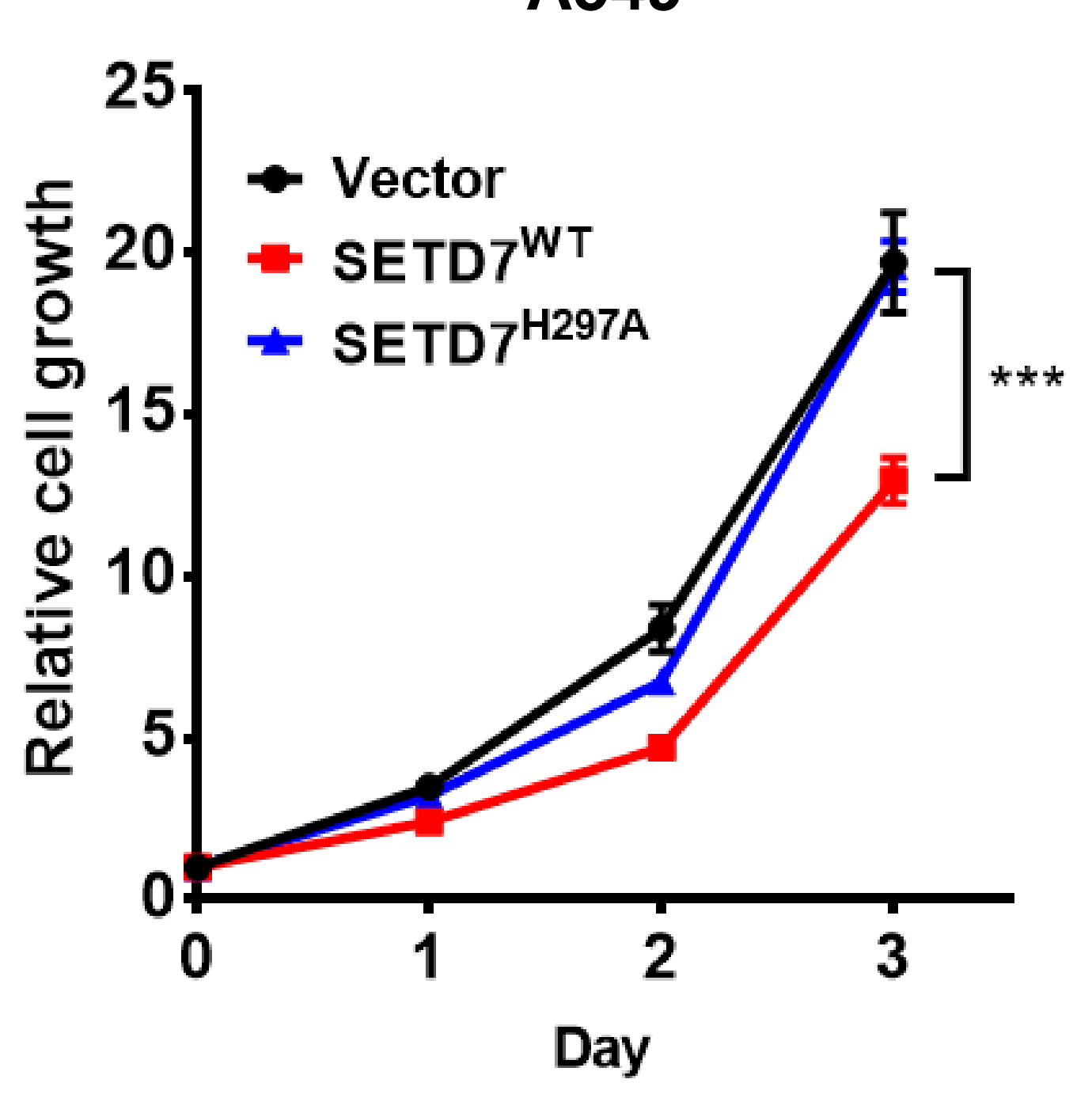

H

A549
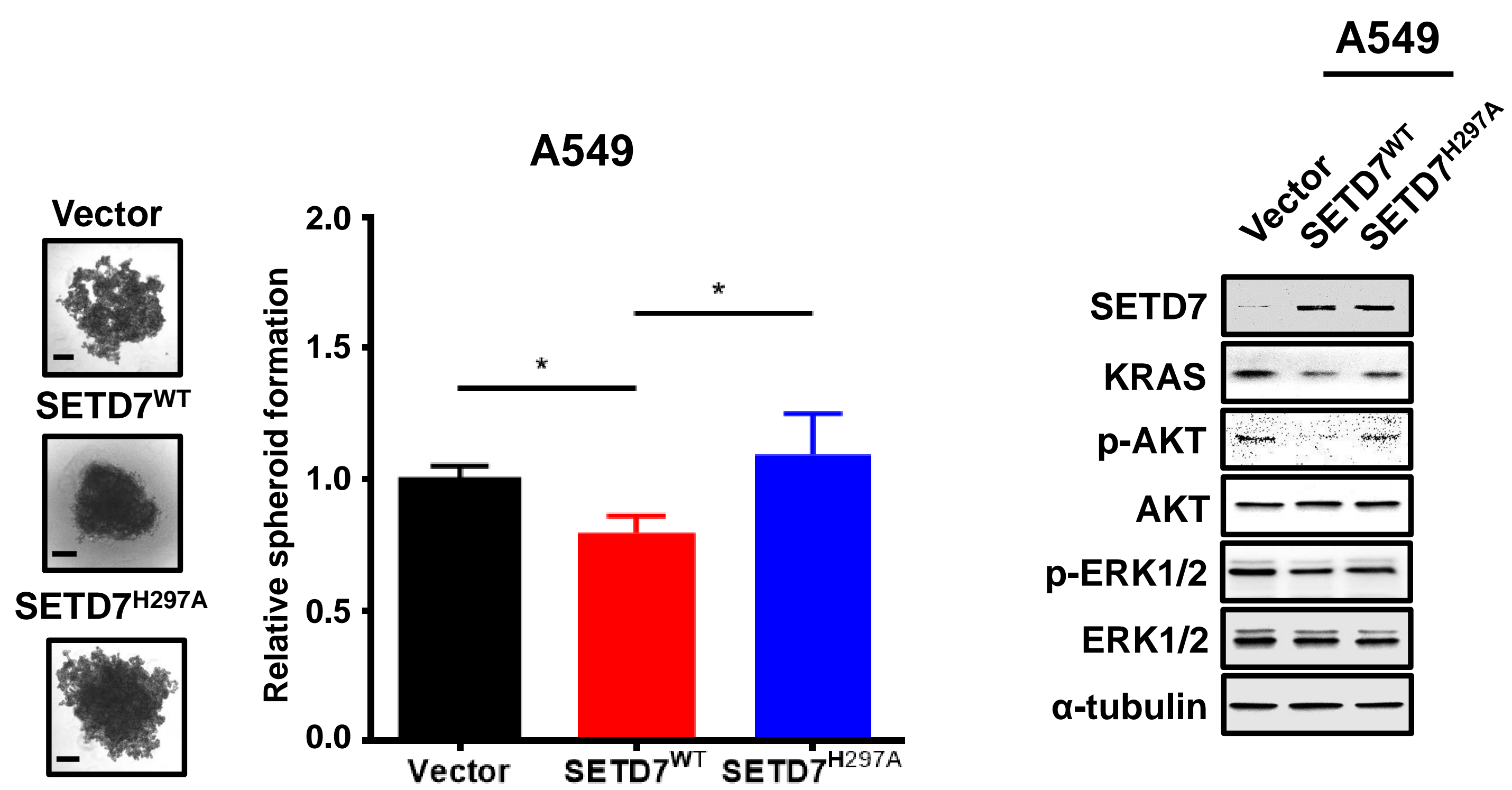


\section{Figure 5}

A

\section{SETD7 consensus sequence} [K/R]-[S/T/A]-K

\begin{tabular}{rrrrrrrrrl}
\hline Position & & -3 & -2 & -1 & 0 & 1 & 2 & 3 & \\
\hline KRAS-4B & 178 & K & K & S & K & T & K & C & 186 \\
HIF-1 & 29 & R & R & S & K & E & S & E & 35 \\
P53 & 369 & L & K & S & K & K & G & Q & 375 \\
TAF10 & 186 & S & K & S & K & D & R & K & 192 \\
E2F1 & 182 & K & K & S & K & N & H & I & 188 \\
DNMT1 & 139 & R & R & S & K & S & D & G & 145 \\
ER & 299 & K & R & S & K & K & N & S & 305 \\
\hline
\end{tabular}

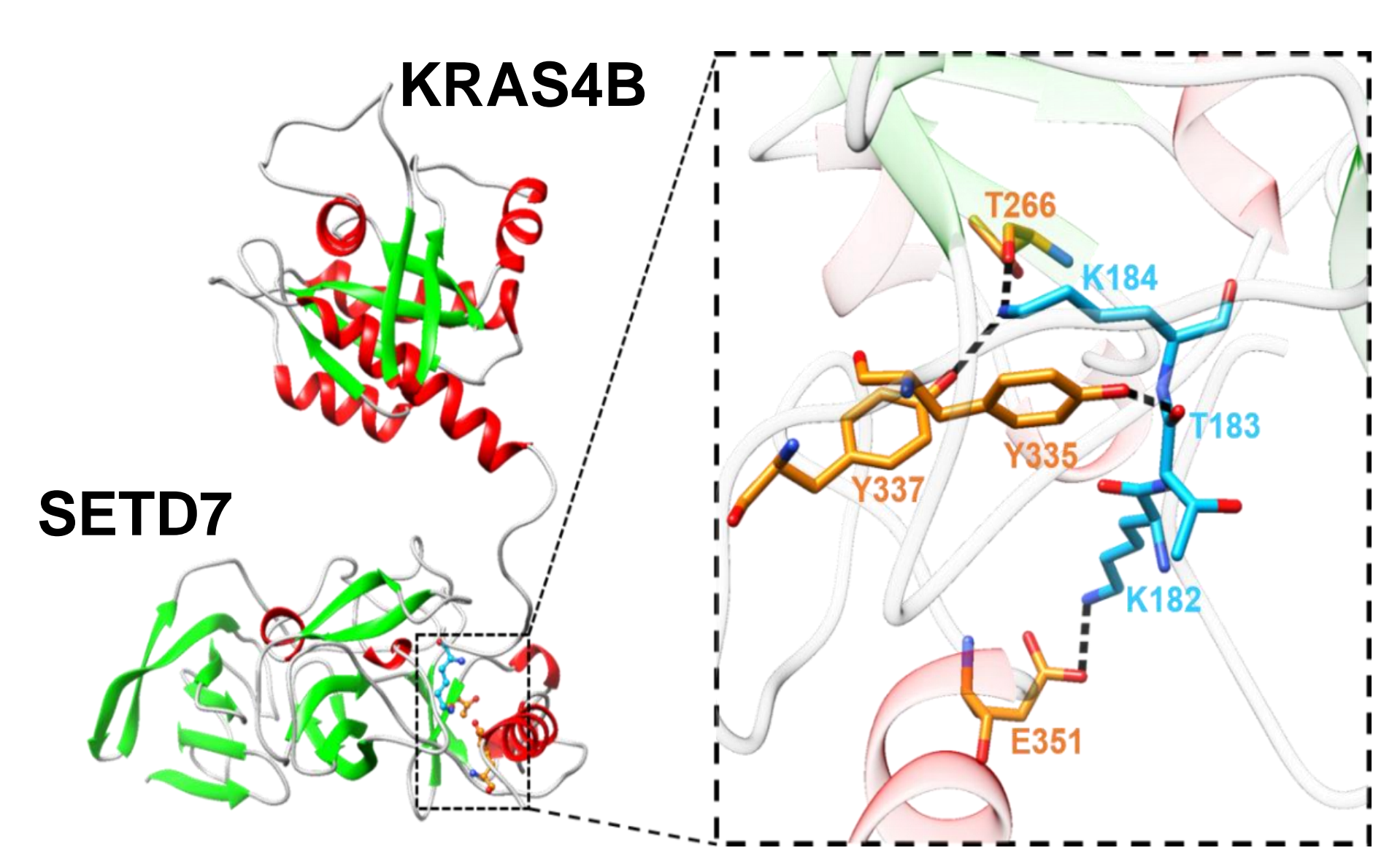

E

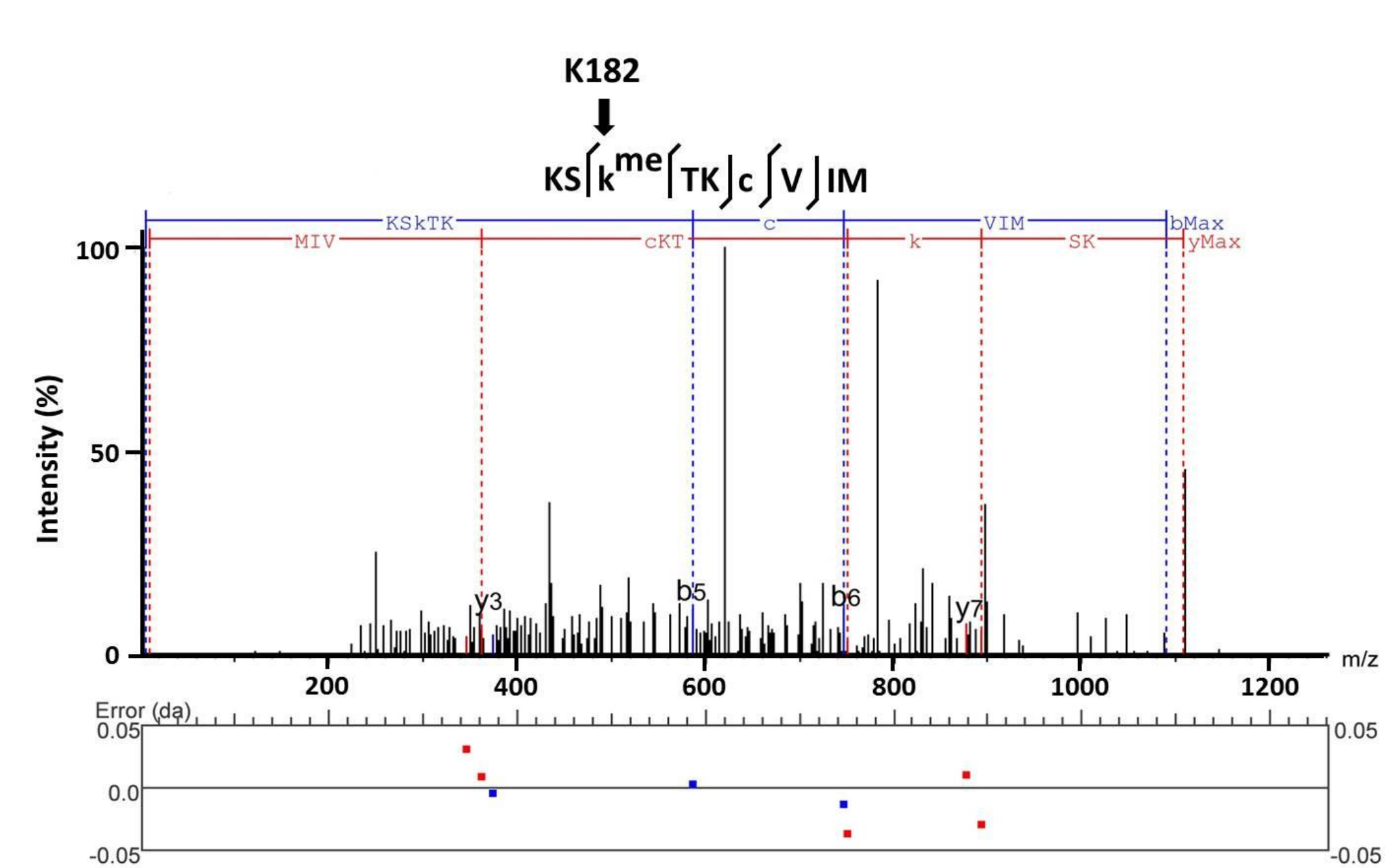

$\mathbf{F}$

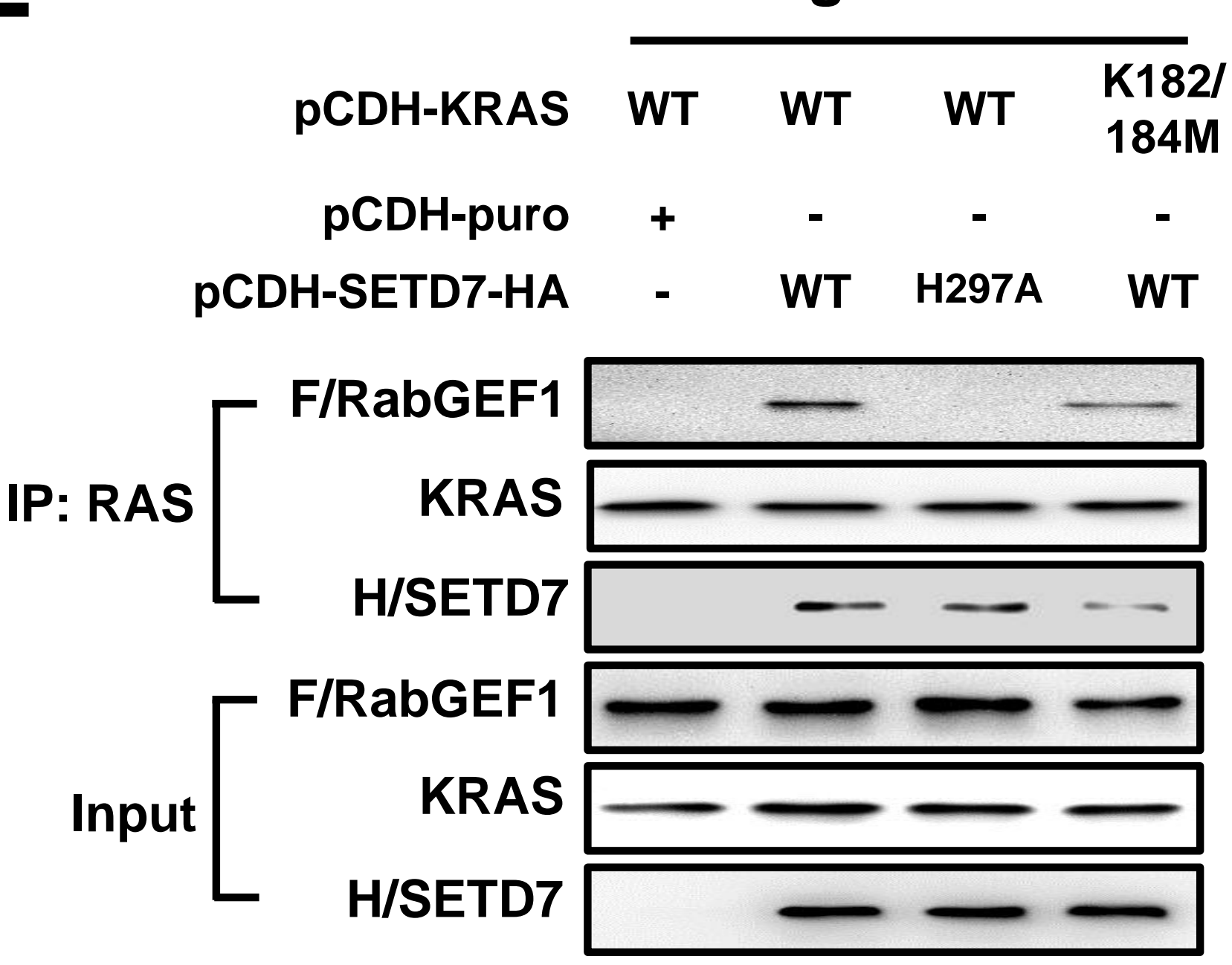

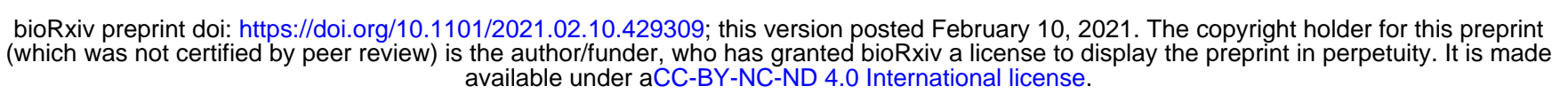

G.

293T-Flag-RabGEF1-OE
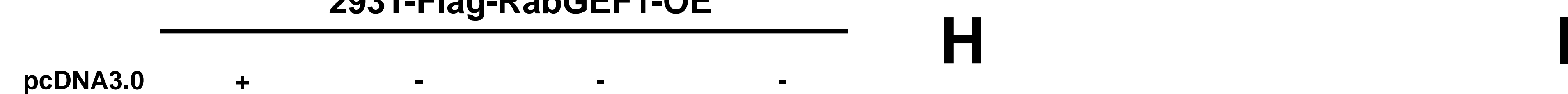

$\mathbf{J}$

\section{T-Flag-RabGEF1-OE}

$\begin{array}{lllll}\text { PCDH-KRAS } & \text { WT } & \text { WT } & \text { K182/184M } & \text { G12D }\end{array}$

CHX (hr): $\quad \begin{array}{llllllllllllllll}0 & 4 & 8 & 12 & 0 & 4 & 8 & 12 & 0 & 4 & 8 & 12 & 0 & 4 & 8 & 12\end{array}$

\begin{tabular}{|c|c|c|c|c|}
\hline RAS & $--\cdots$ & $-\cdots$ & $-\cdots$ & $-\cdots$ \\
\hline H/SETD7 & & ---- & --- & --- \\
\hline F/RabGEF1 & $-\infty-\cdots$ & ---- & --- & --- \\
\hline$\alpha$-tubu & - & ---- & ---- & $-\infty$ \\
\hline
\end{tabular}

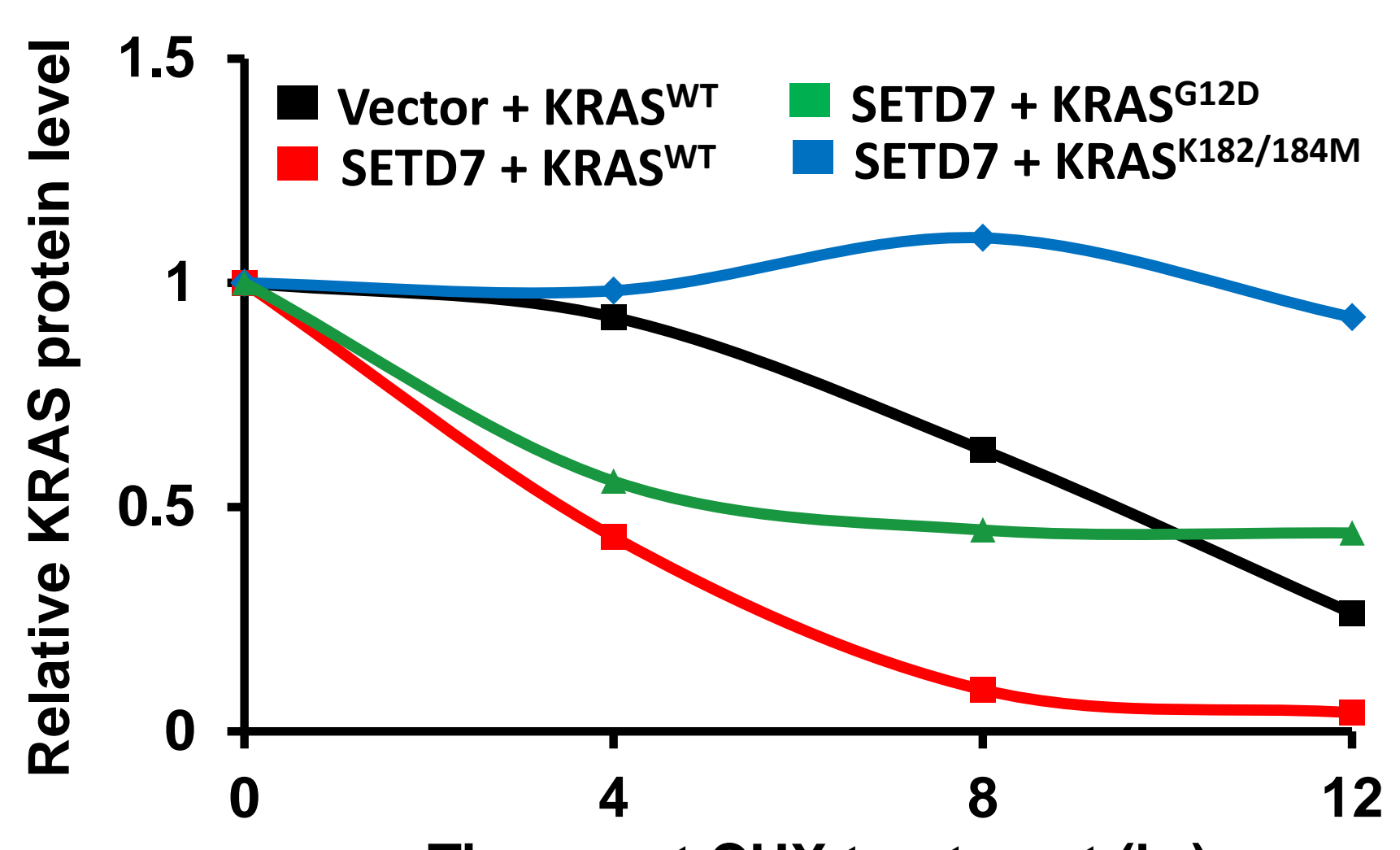

Time post $\mathrm{CHX}$ treatment $(\mathrm{hr})$
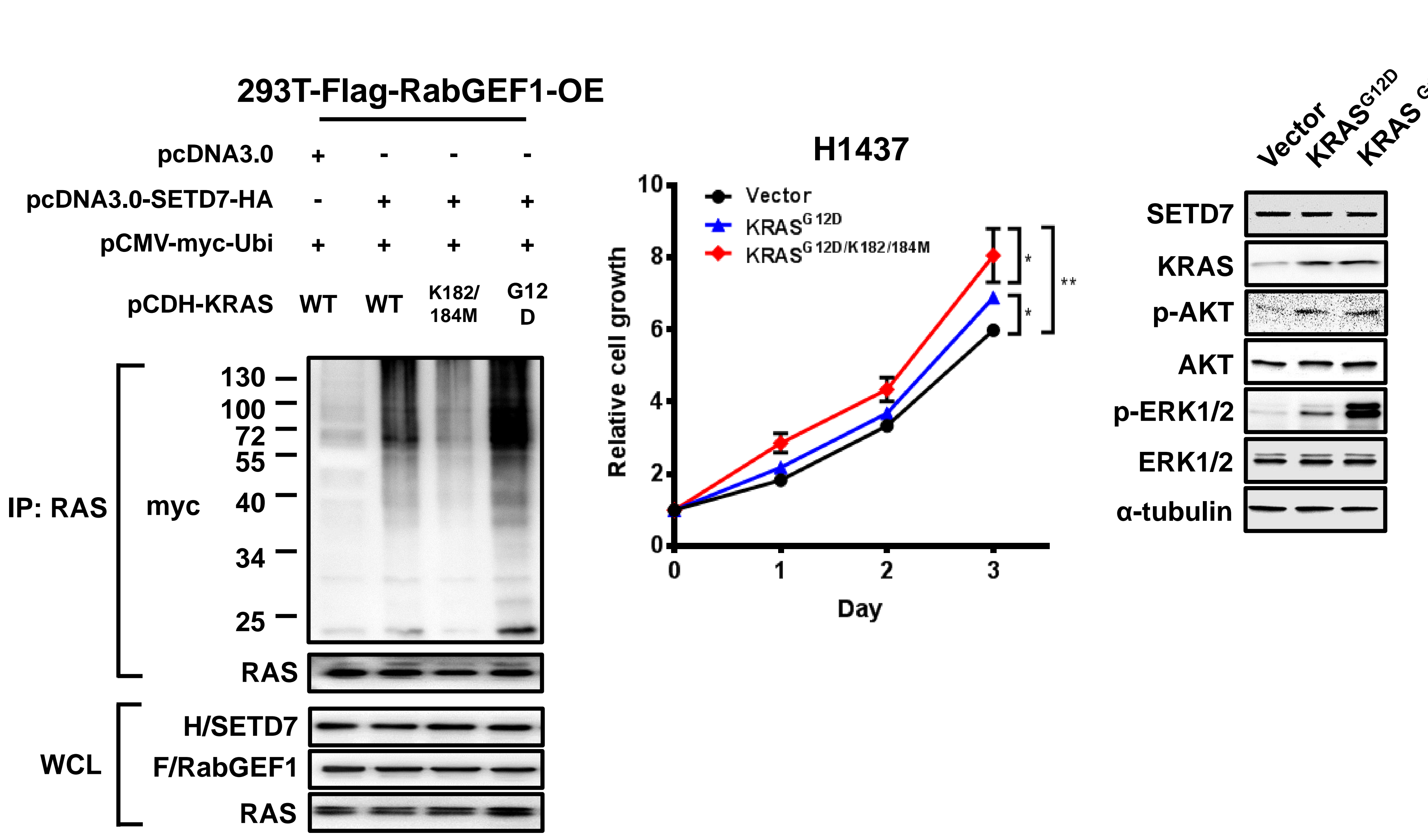
A

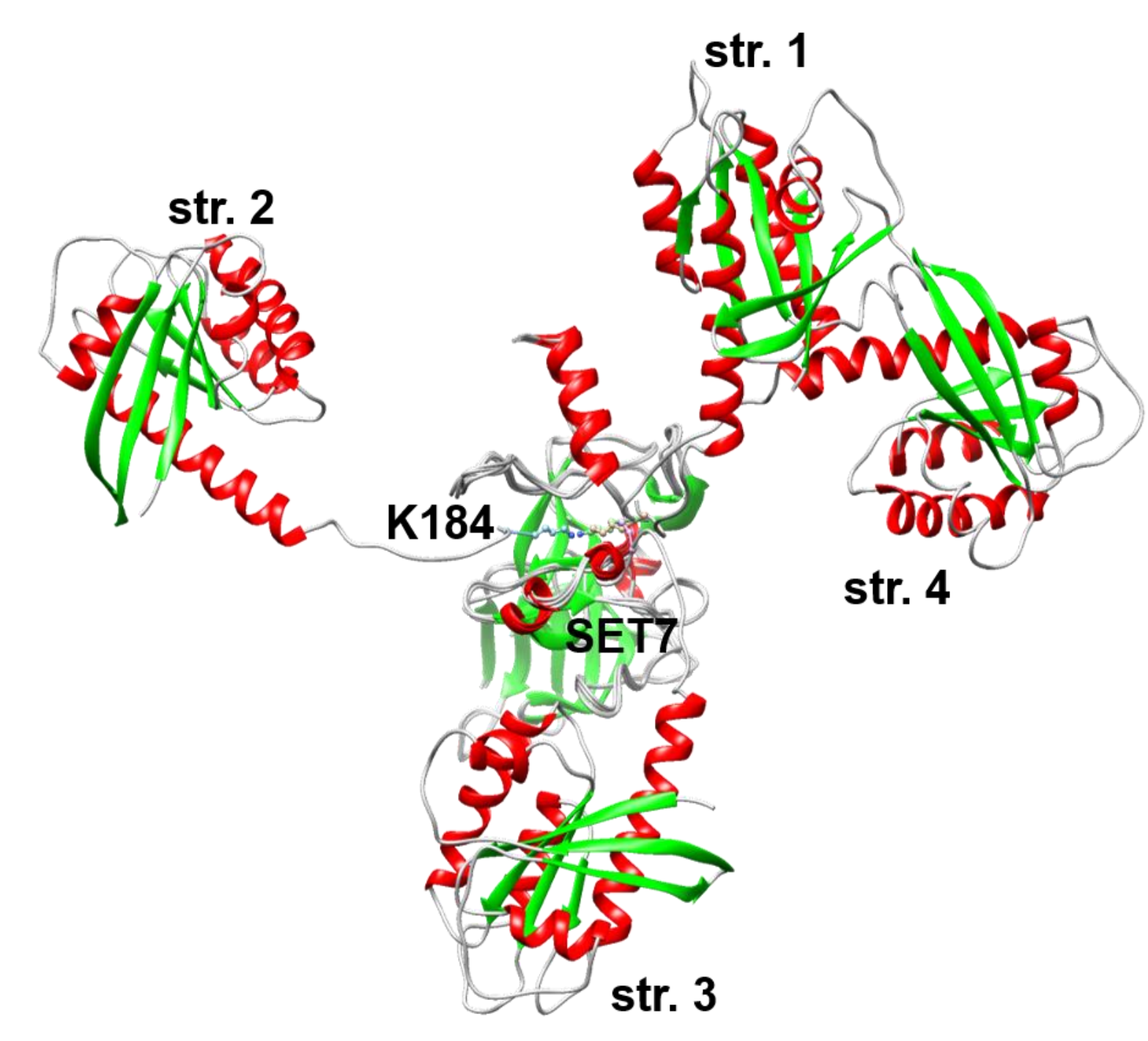

C
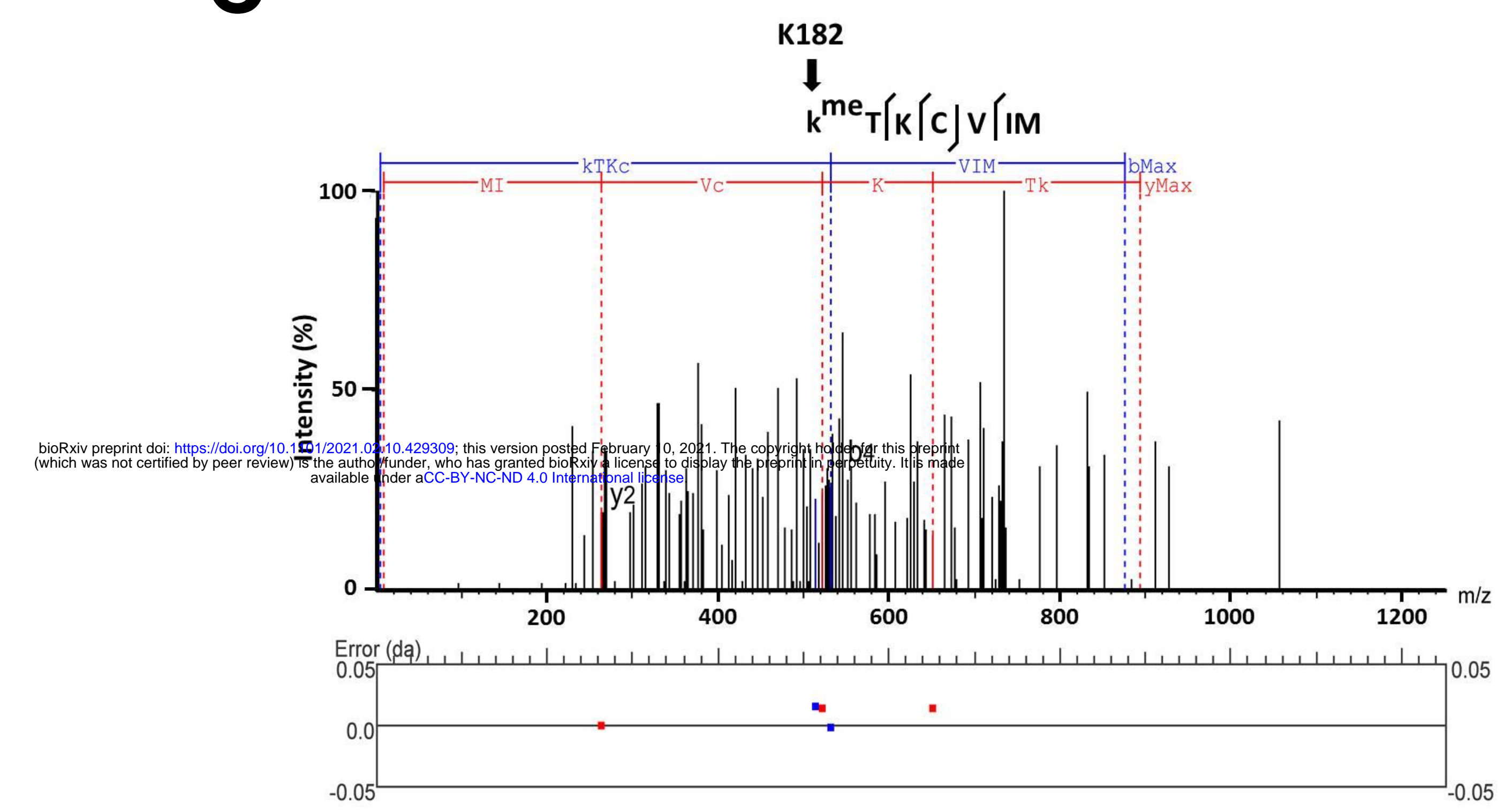

E

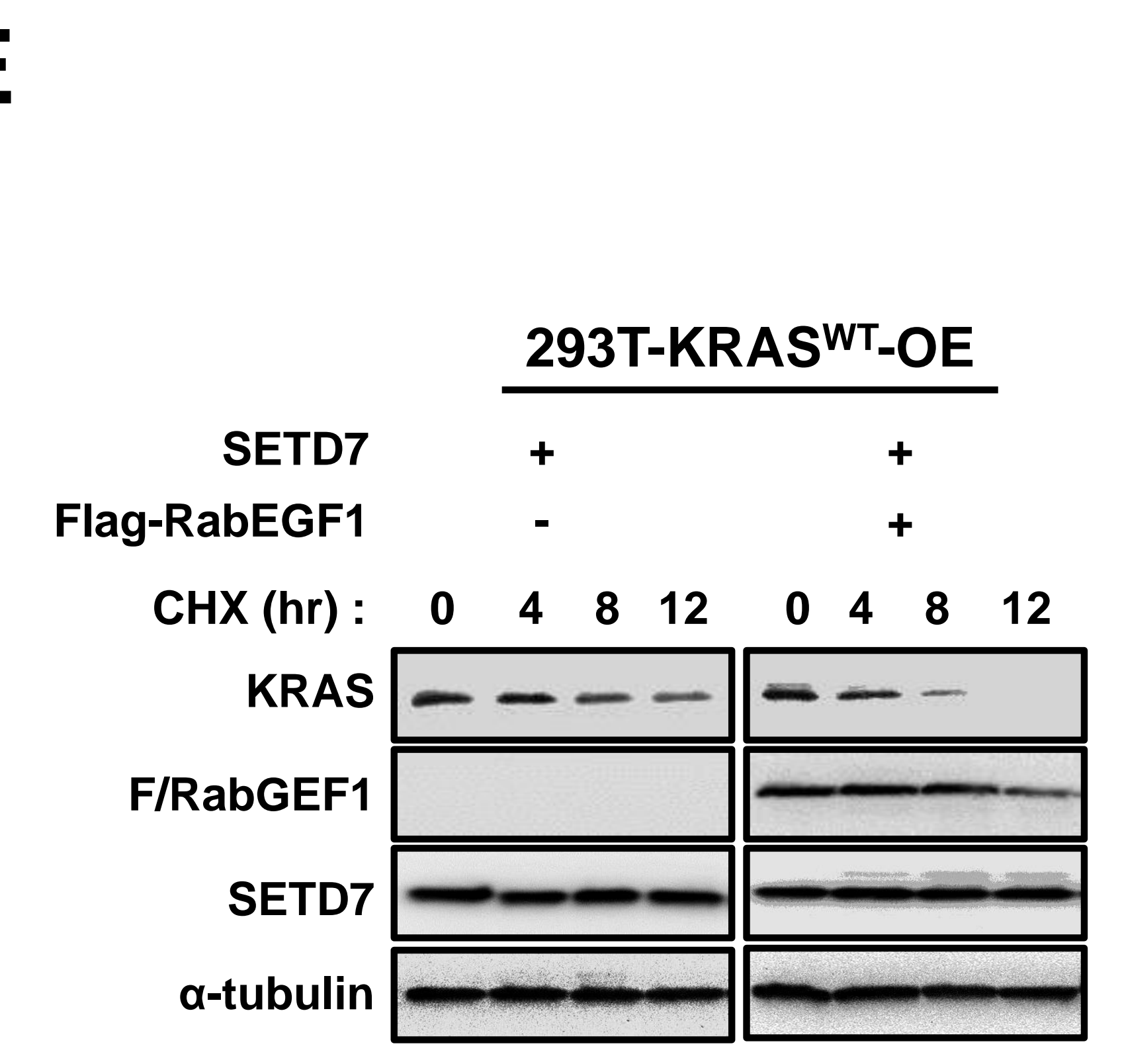

F

\section{B}
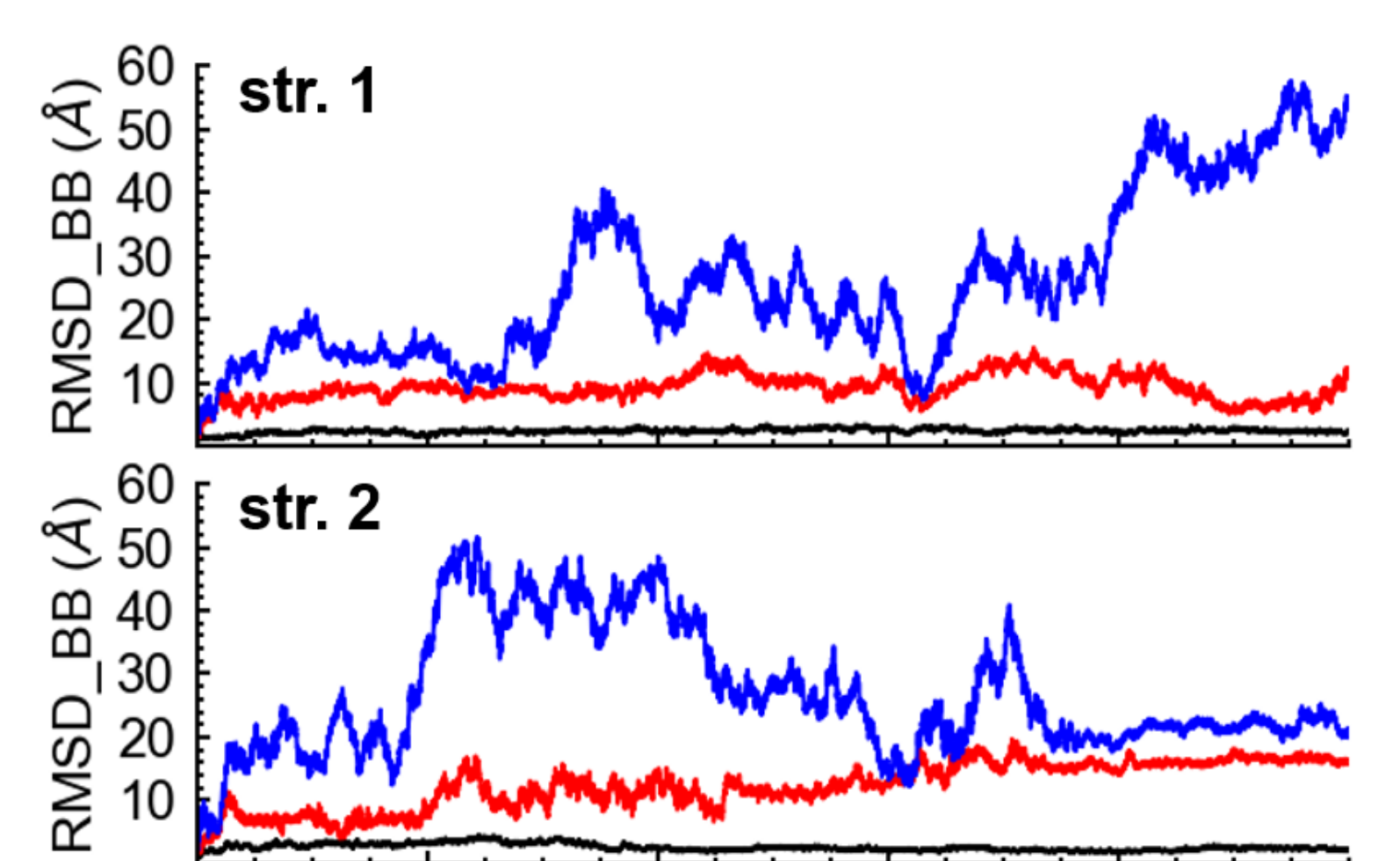

它 50 str. 3

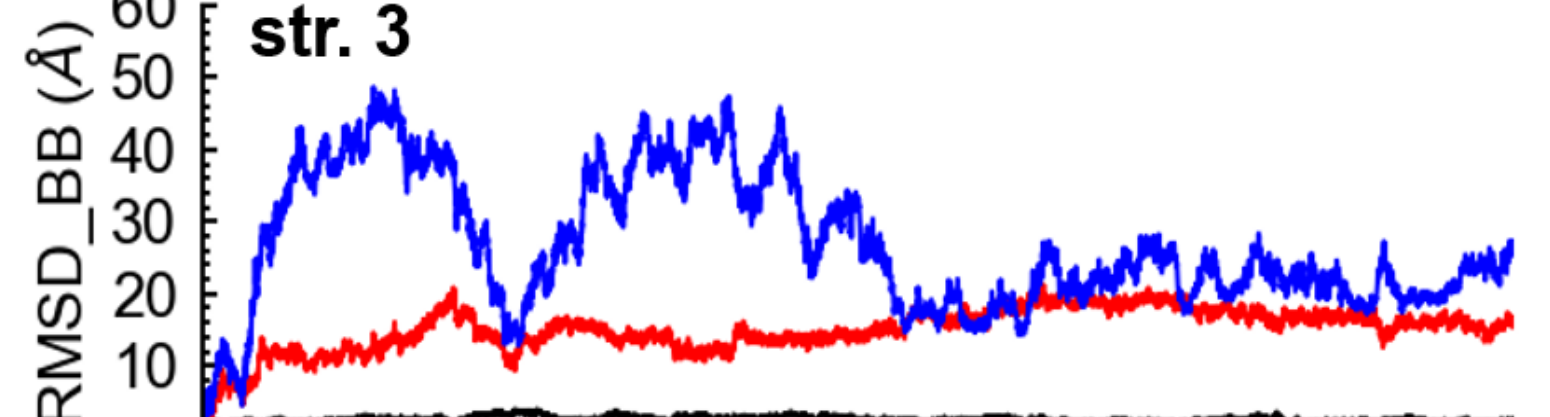

$\sum_{x} 10$ N

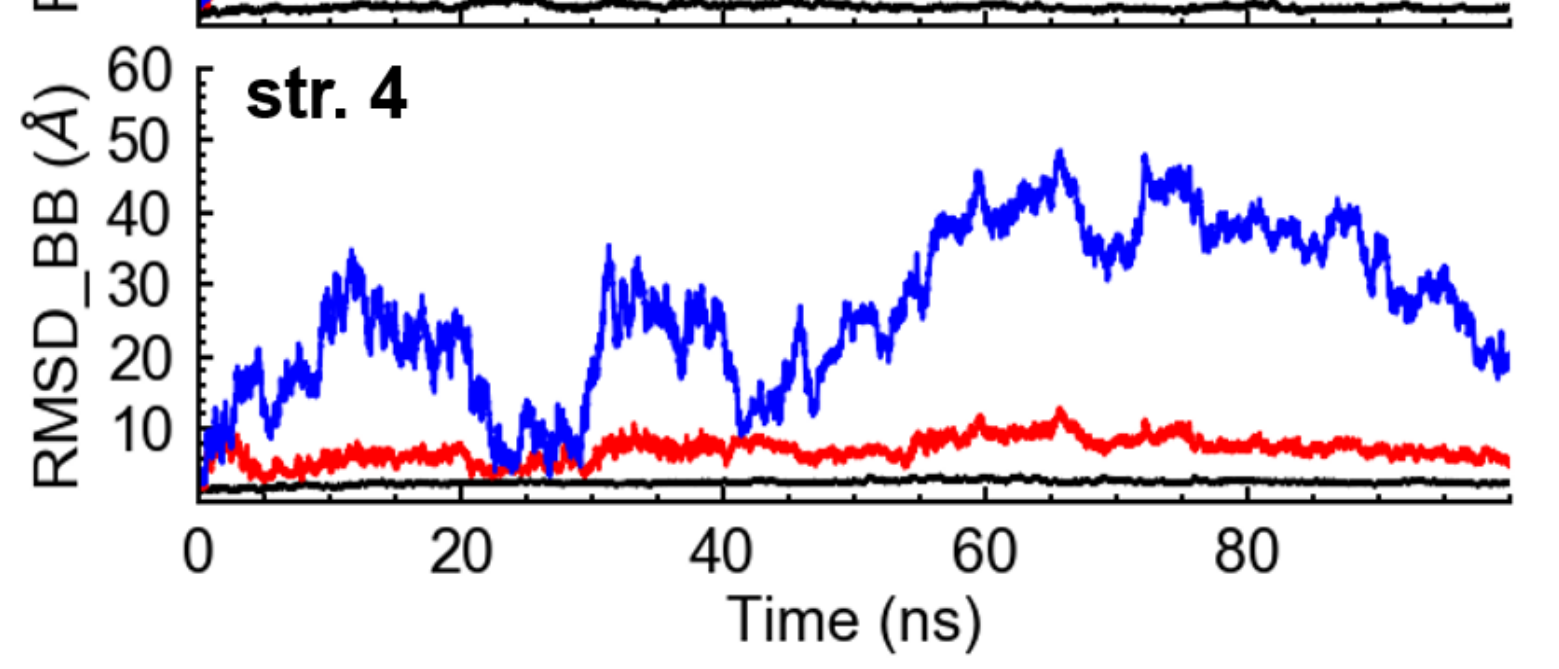

D

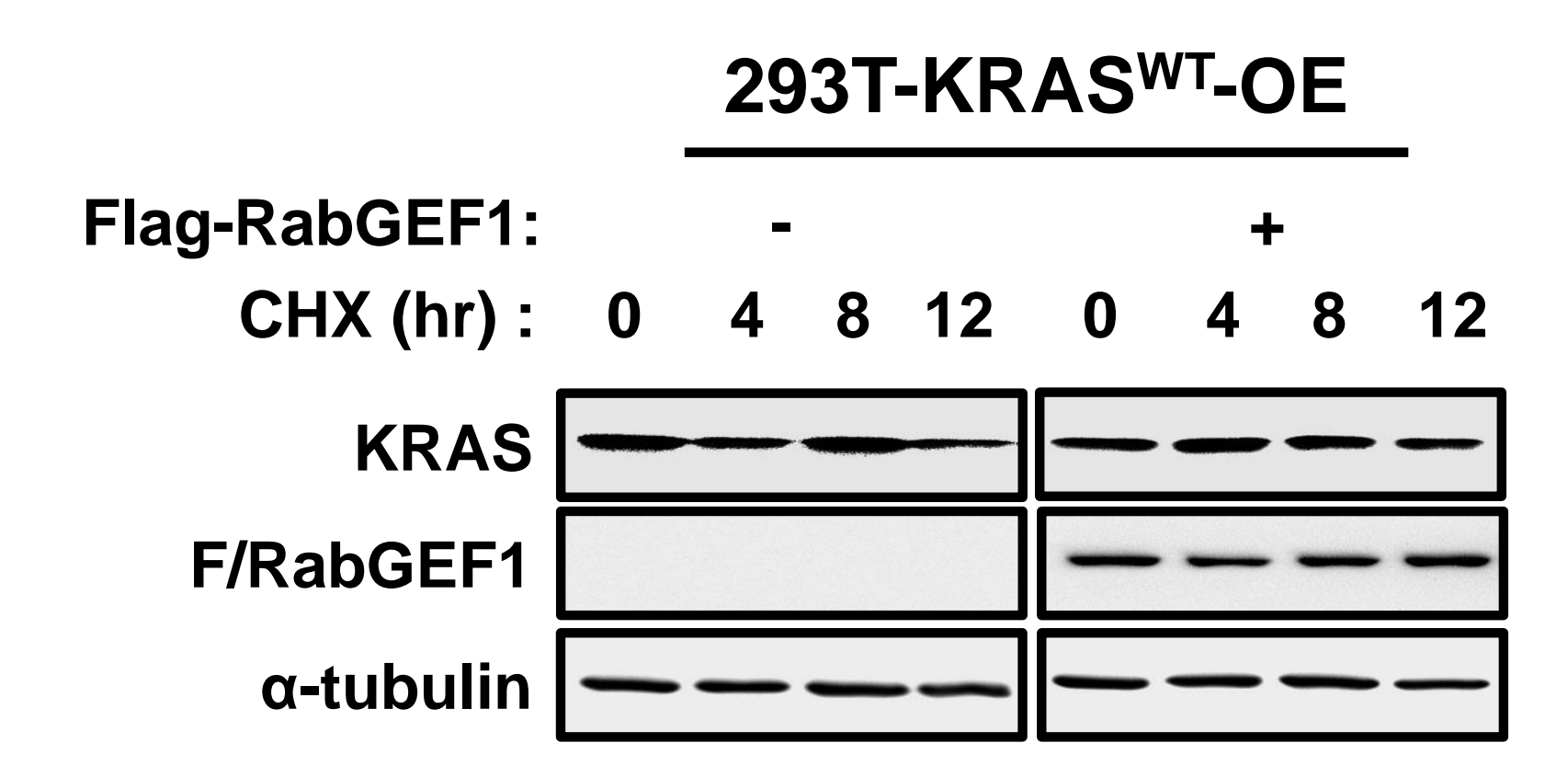

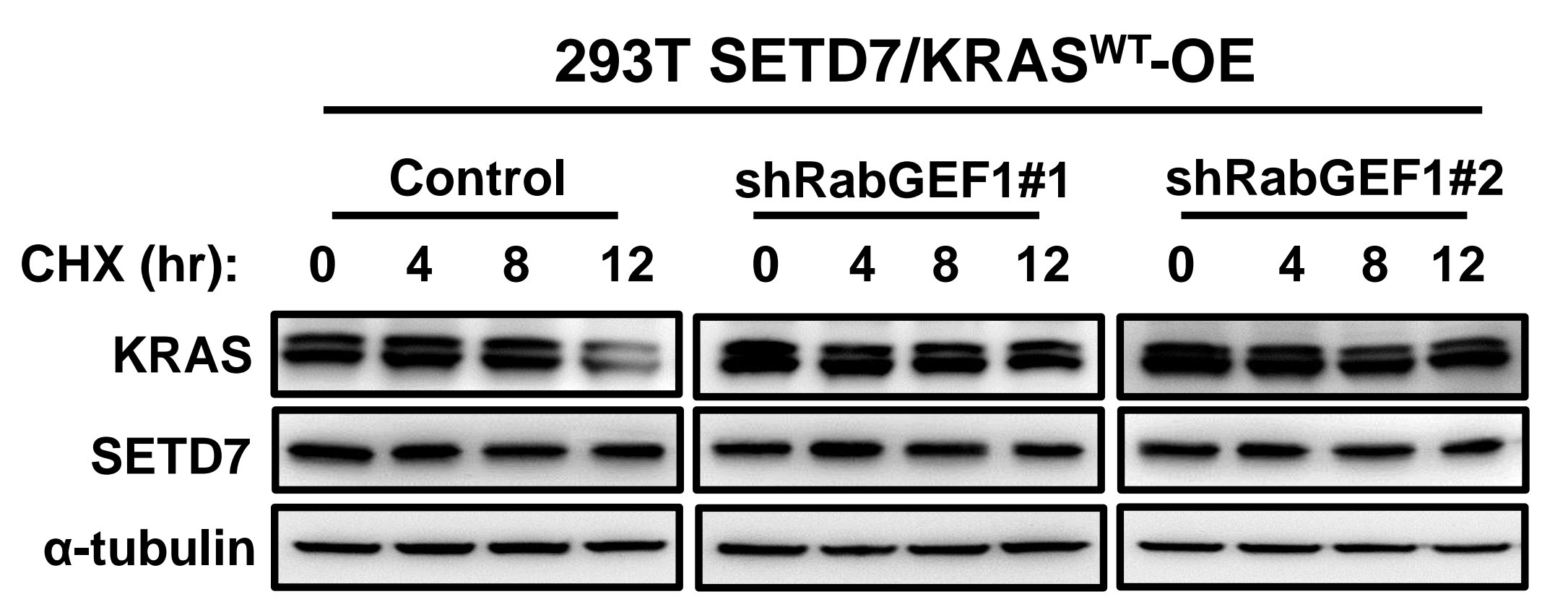

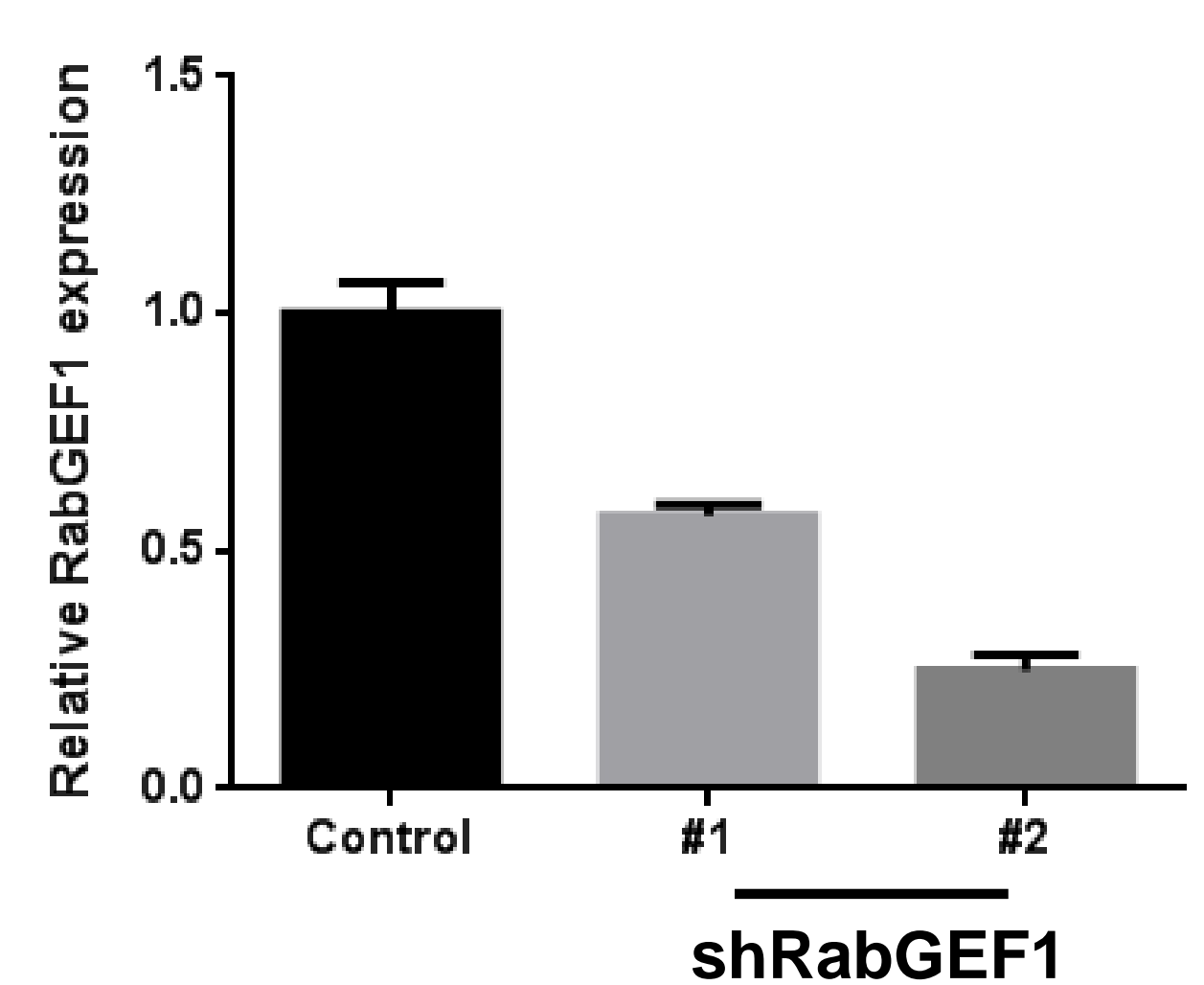


Figure 6

A
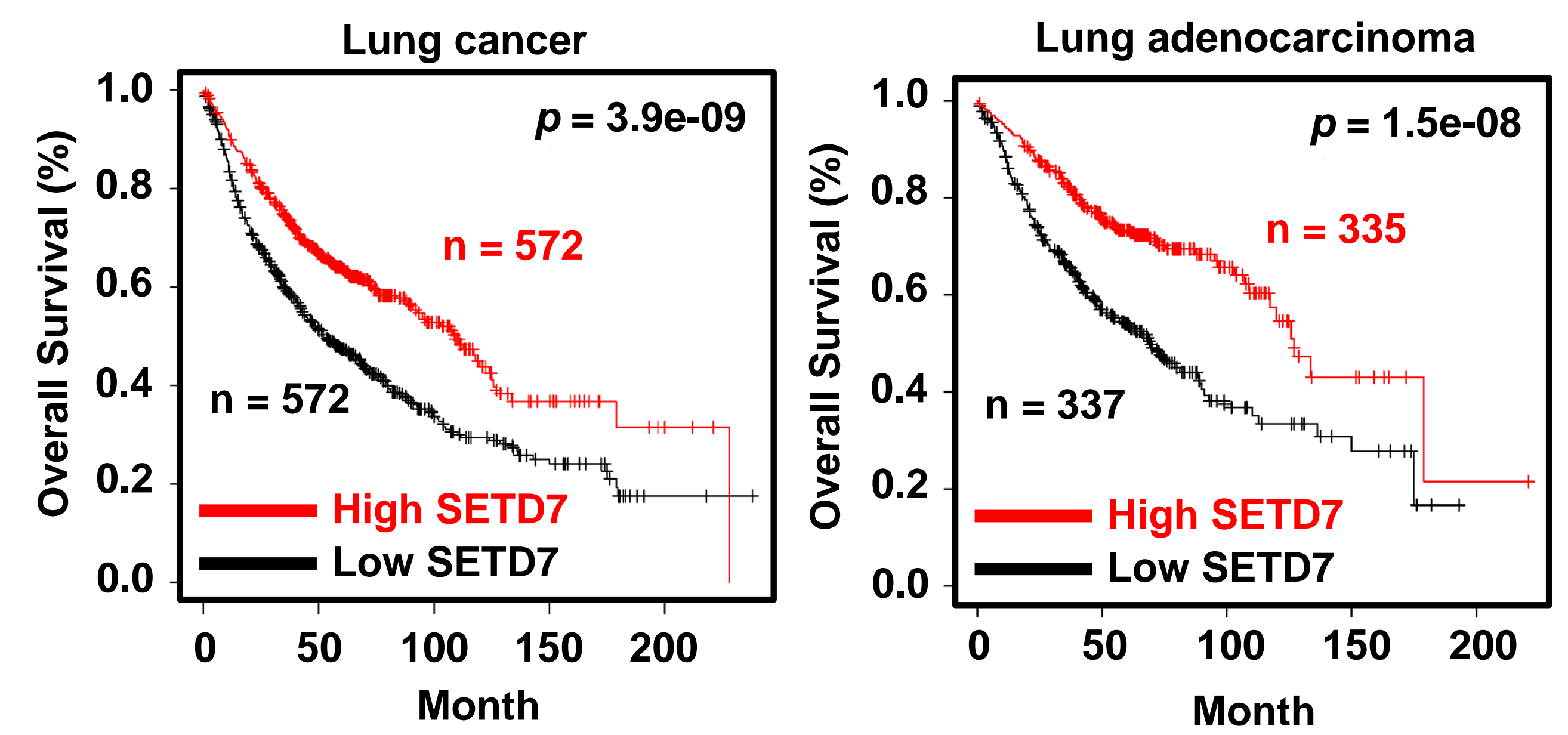

B

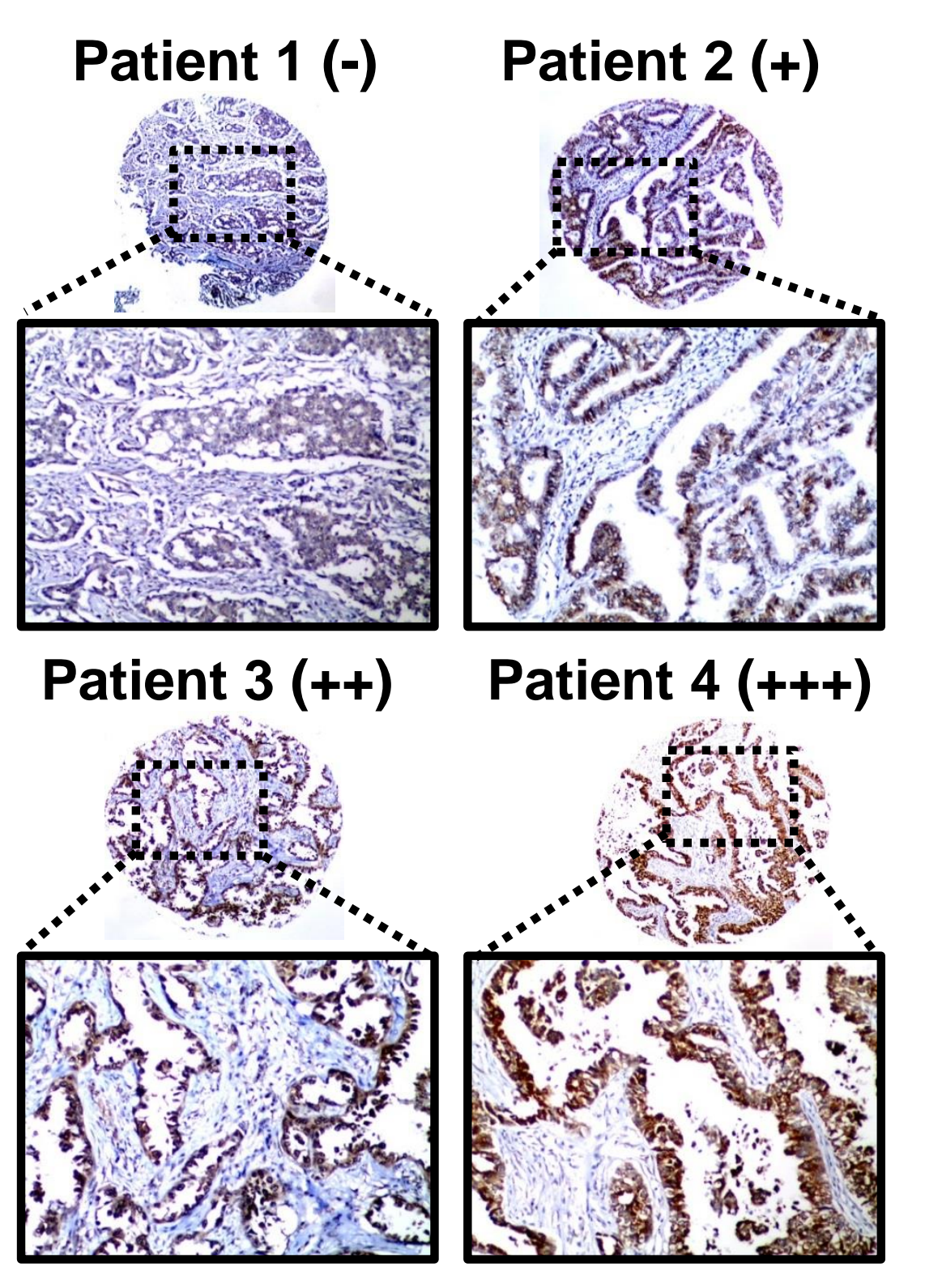

C

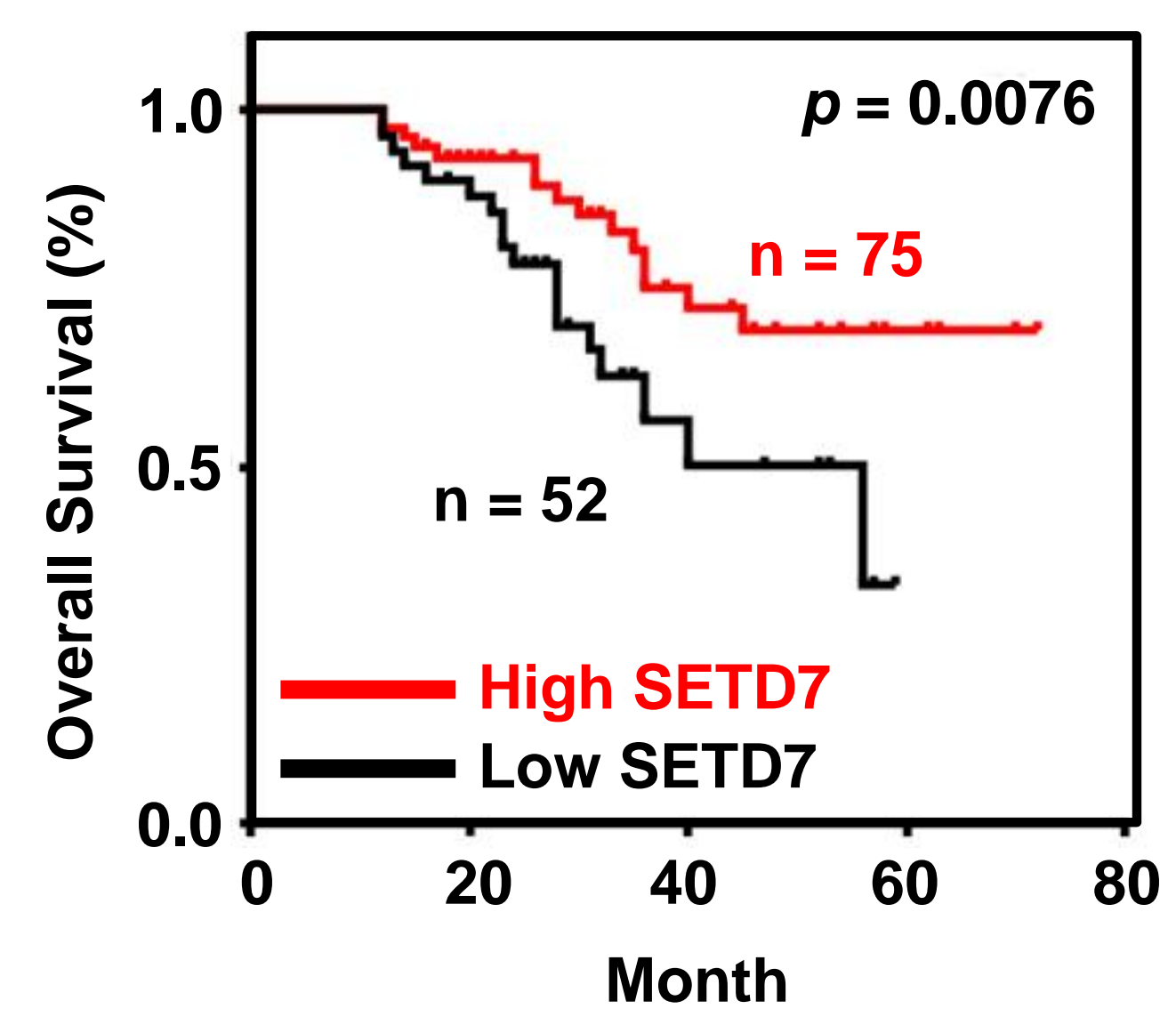




\section{Figure S6}
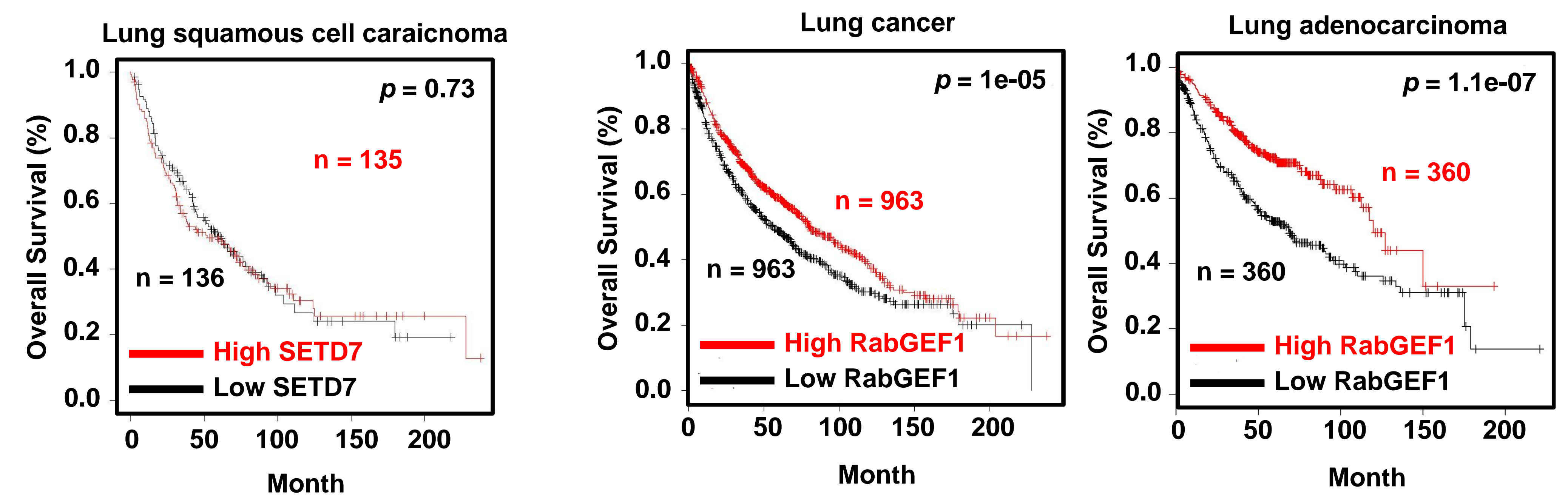

C

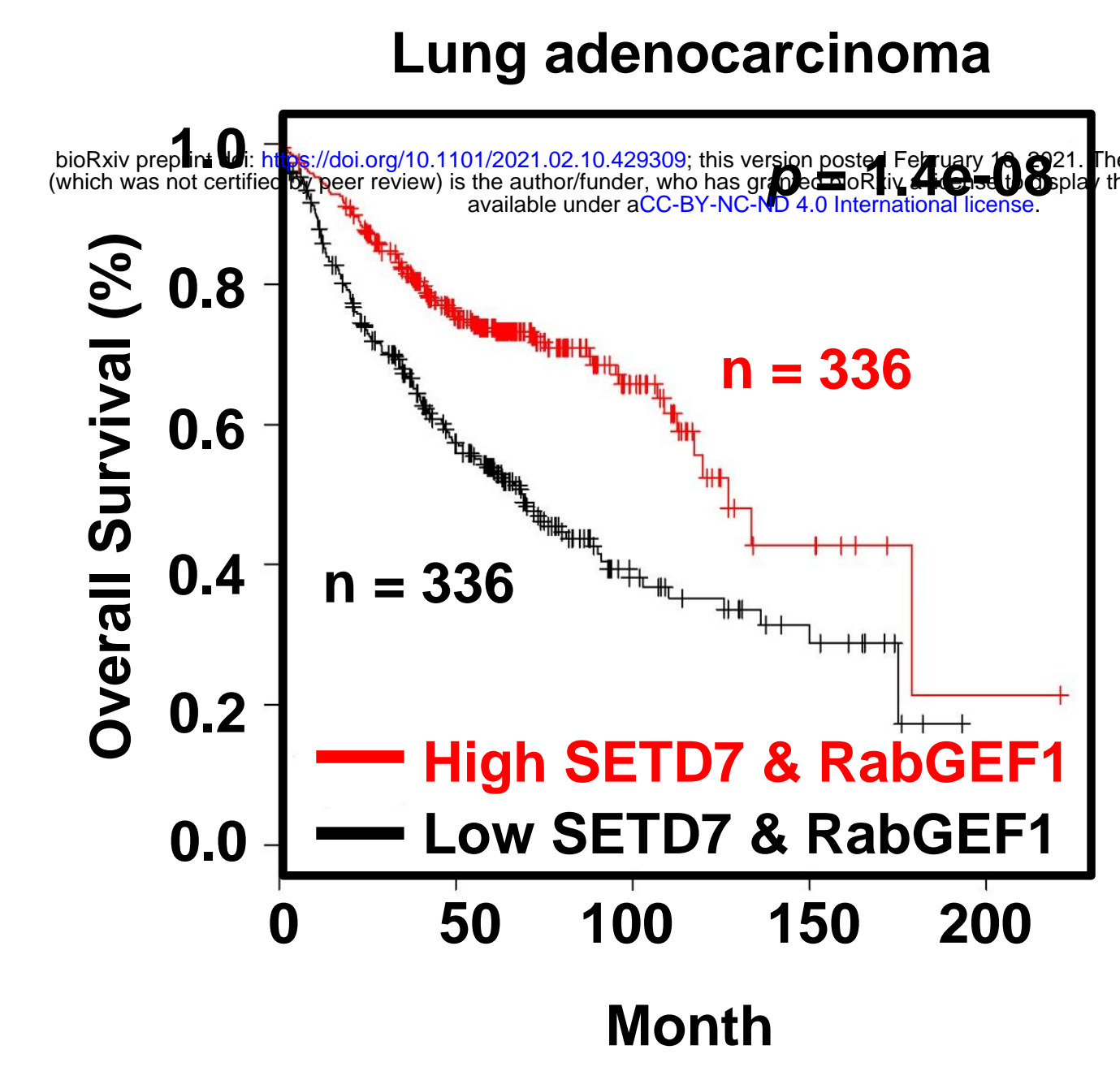

$\mathbf{F}$

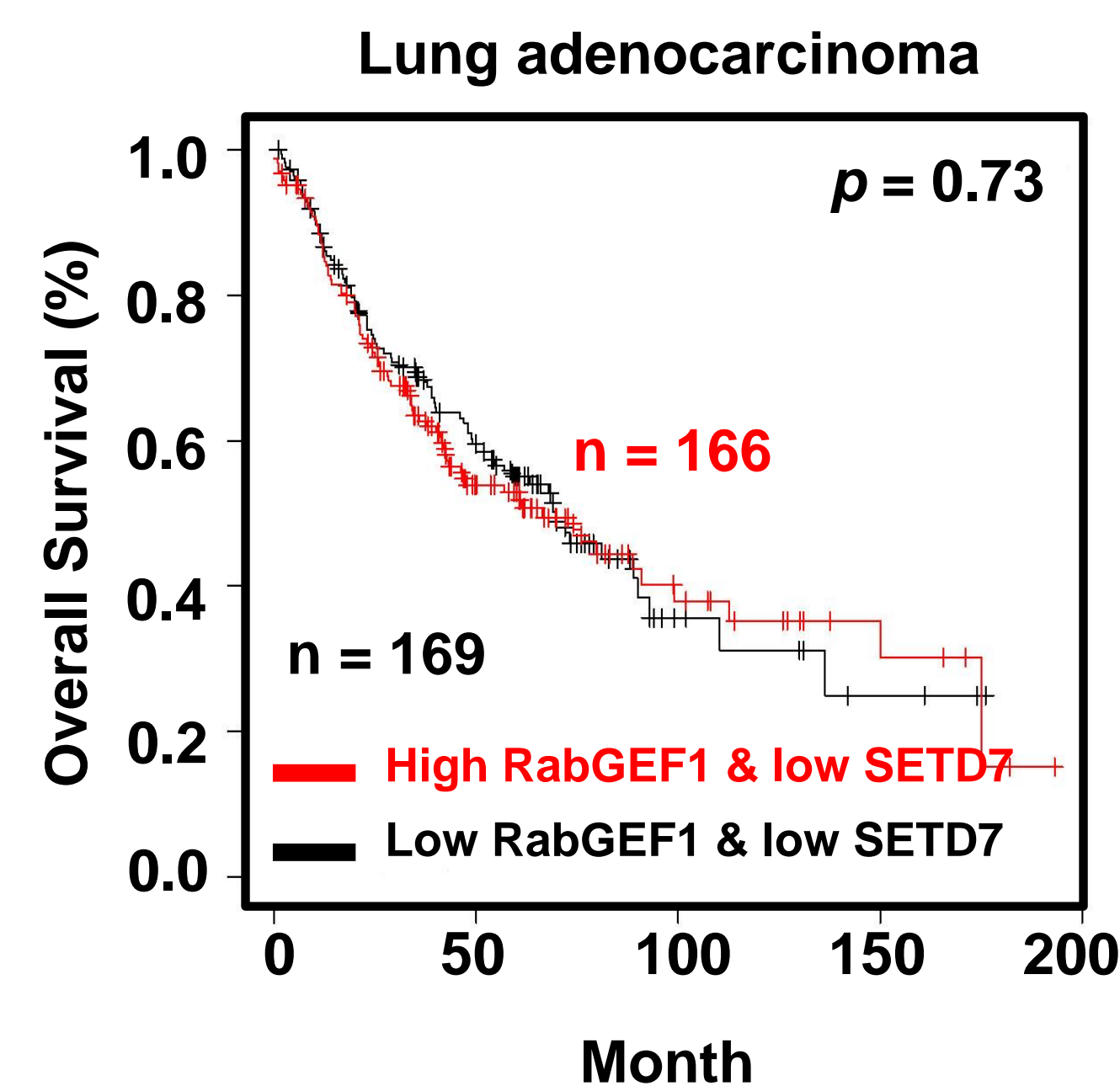

D

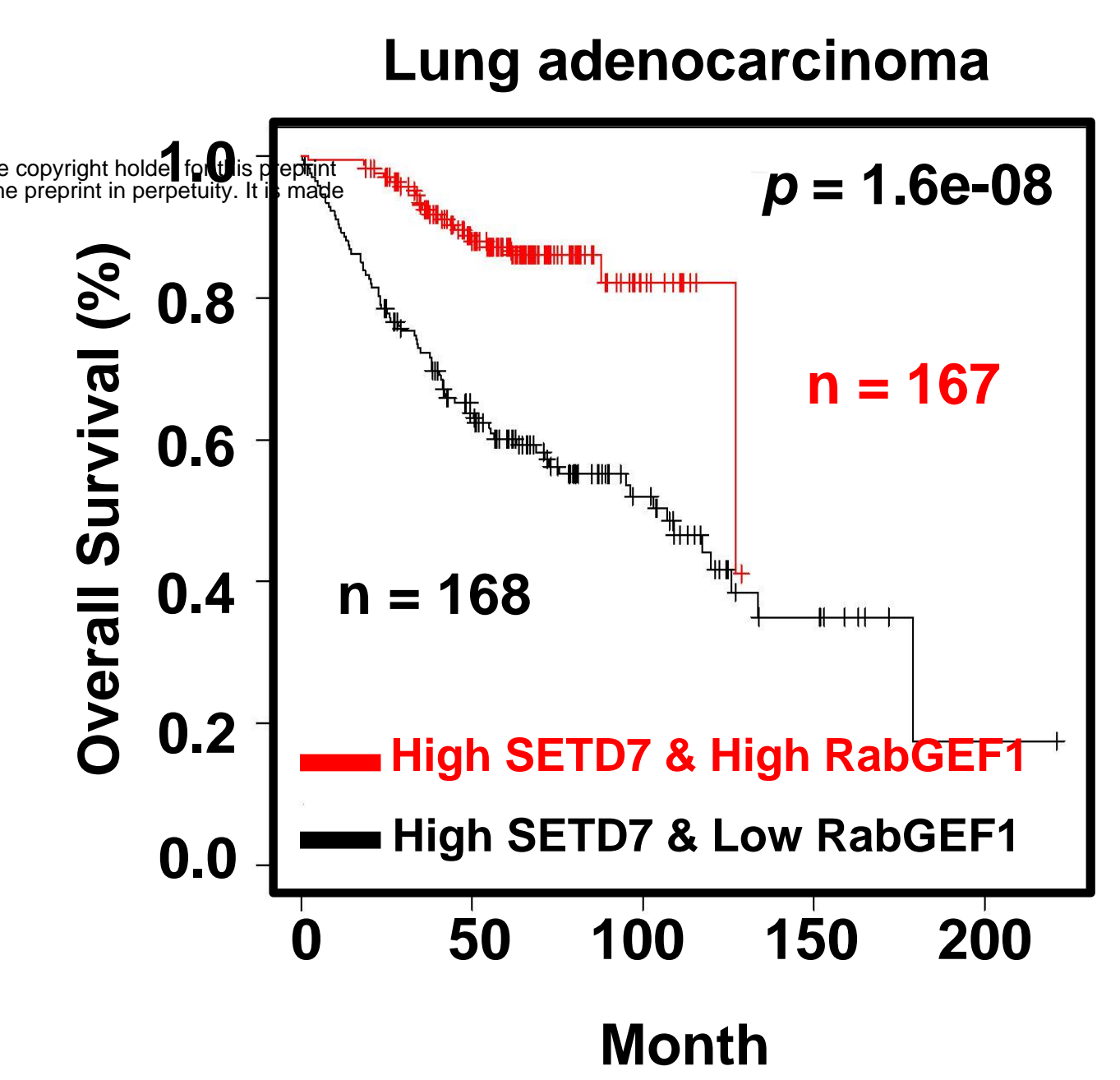

G

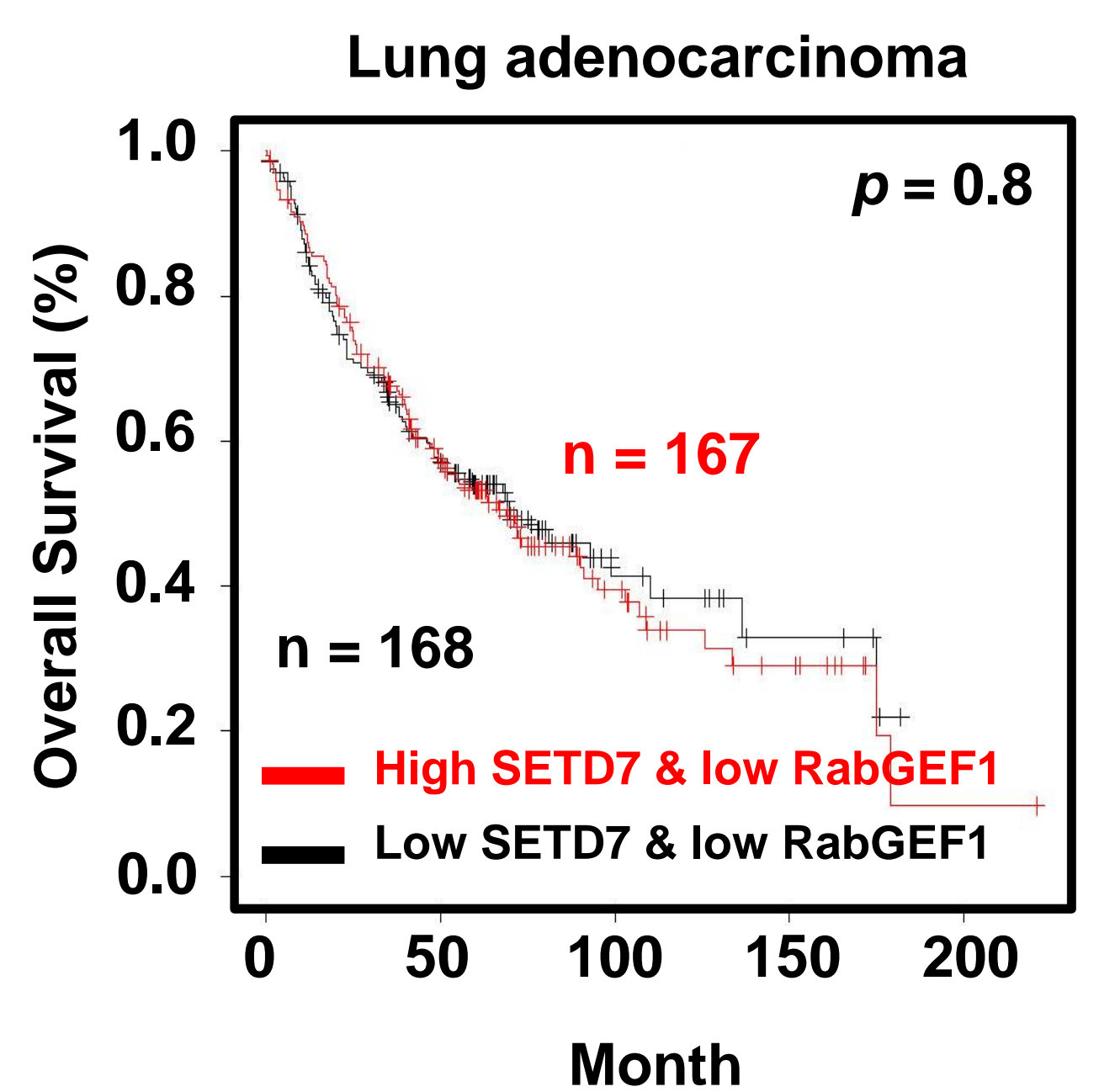

E

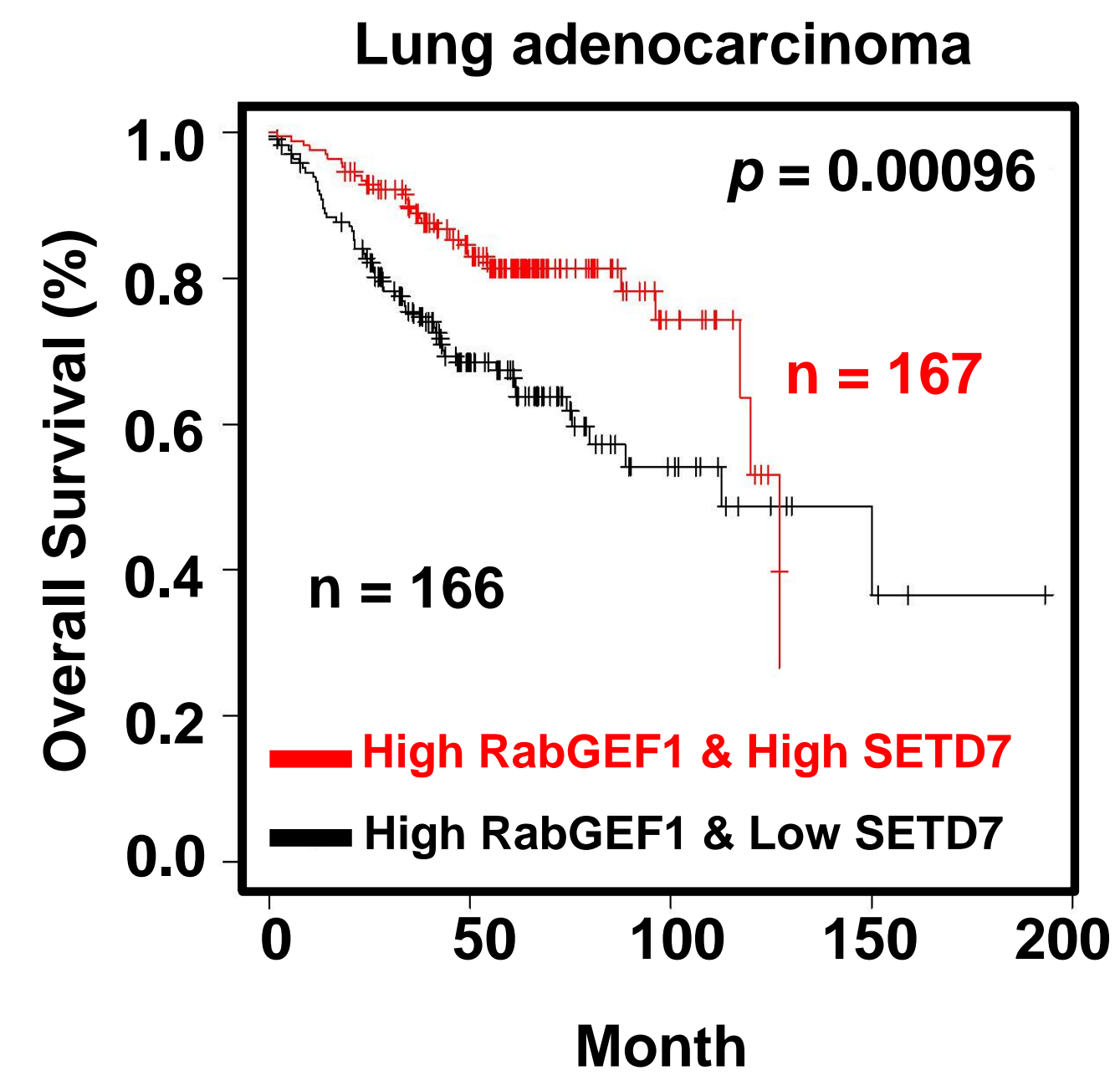




\section{Figure 7}

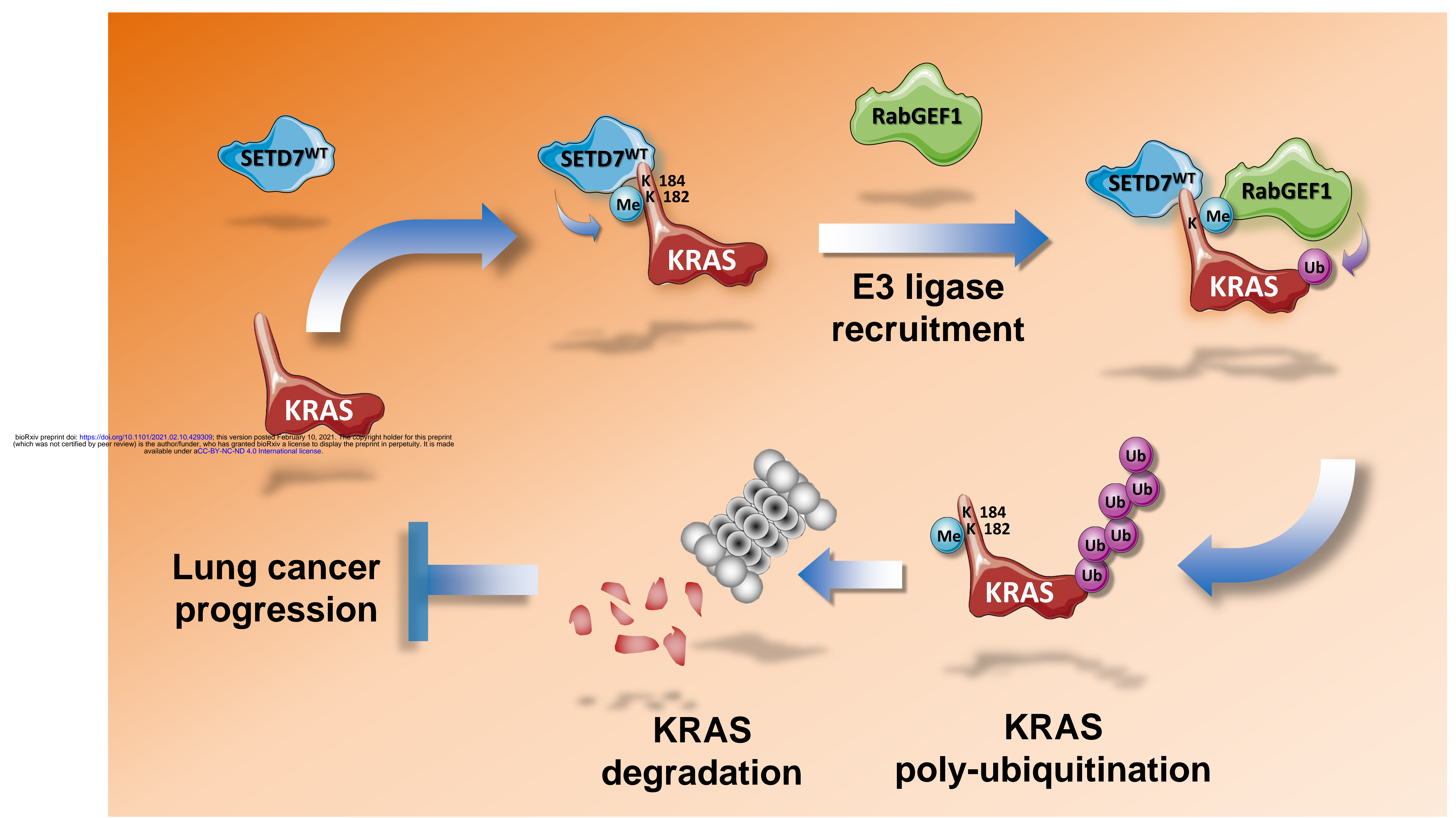

